\title{
AN ENERGY INEQUALITY FOR HIGHER ORDER LINEAR PARABOLIC OPERATORS AND ITS APPLICATIONS $\left({ }^{1}\right)$
}

\author{
BY \\ DAVID ELLIS
}

\begin{abstract}
A generalization of the classical energy inequality is obtained for evolution operators $(\partial / \partial t) I-H(t) \Lambda^{2 k}-J(t)$, associated with higher order linear parabolic operators with variable coefficients. Here $H(t)$ and $J(t)$ are matrices of singular integral operators. The key to the result is an algebraic inequality involving matrices similar to the symbol of $H(t)$ having their eigenvalues contained in a fixed compact subset of the open left-half complex plane. Then a sharp estimate on the norms of certain imbedding maps is obtained. These estimates along with the energy inequality is applied to the Cauchy problem for higher order linear parabolic operators restricted to slabs in $R^{n+1}$.
\end{abstract}

1. Introduction. We present a Hilbert space treatment for proving existence and uniqueness in the Cauchy problem for a general linear $2 k$-parabolic differential operator. We shall follow in rough outline the Hilbert space approach to the Cauchy problem for parabolic operators of the form

$$
P=\frac{\partial}{\partial t}-L(t) \equiv \frac{\partial}{\partial t}-\sum_{|\alpha| \leqq 2 k} a_{\alpha}(x, t) D^{\alpha},
$$

where $L(t)$ is uniformly strongly elliptic on $R^{n}$ (here $x=\left\langle x_{1}, \ldots, x_{n}\right\rangle$, $\alpha=\left\langle\alpha_{1}, \ldots, \alpha_{n}\right\rangle,|\alpha|=\alpha_{1}+\cdots+\alpha_{n}$, and $\left.D^{\alpha}=\left((1 / i) \partial / \partial x_{1}\right)^{\alpha_{1}} \cdots\left((1 / i) \partial / \partial x_{n}\right)^{\alpha}\right)^{\alpha}$, as given in [5]. As in [5] we shall make use of the Hilbert spaces $\mathscr{H}^{r, s}\left(\equiv \mathscr{B}_{2, k}\right.$ in the notation of [4, Chapter II], where $k(\xi, \tau)=k_{r, s}(\xi, \tau)$ is the temperate weight function defined for $\langle\xi, \tau\rangle=\left\langle\xi_{1}, \ldots, \xi_{n}, \tau\right\rangle \in R^{n+1}$ by $k_{r, s}(\xi, \tau)=Q^{r}(\xi, \tau) q^{s}(\xi)$. Here $q(\xi)=\left\{1+|\xi|^{2}\right\}^{1 / 2}$, with $|\xi|^{2}=\sum_{j=1}^{n} \xi_{j}^{2}$, is the usual elliptic weight function in $R^{n}$ and $\left.Q(\xi, \tau)=\left\{\tau^{2}+q^{4 k}(\xi)\right\}^{1 / 4 k}\right)$. $H^{s}$ is the usual Sobolev space on $R^{n}$.

We assume $P$ is of the form

$$
P\left(x, t, D, D_{t}\right)=\sum_{|\alpha|+2 k j \leqq 2 k m} a_{\alpha, j}(x, t) D^{\alpha} D_{t}^{j}
$$

Received by the editors October 27, 1970.

AMS 1970 subject classifications. Primary 35K30, 44A25; Secondary 15A42.

Key words and phrases. Partial differential operator, evolution operator, singular integral operator, symbol of an operator, test function, temperate weight function, compact support, Sobolev space.

(1) The research for this paper was supported by the Air Force Office of Scientific Research under contract number AFOSR 537-67. 
with $a_{0, m}$ nonvanishing, and that the functions $\left\{a_{\alpha, j}(x, t):|\alpha|+2 k j \leqq 2 k m\right\}$ belong to the class $C_{B}^{\infty}\left(R^{n+1}\right)$ of complex valued functions having bounded (and therefore continuous) partial derivatives of all orders for all $\langle x, t\rangle \in R^{n+1}$. Moreover, we assume $P$ is uniformly $2 k$-parabolic on $R^{n+1}$, i.e., there exists $\delta>0$ such that

$$
P_{0}(x, t ; \xi, z) \equiv \sum_{|\alpha|+2 k j=2 k m} a_{\alpha, j}(x, t) \xi^{\alpha} z^{j}=0
$$

for $\langle x, t\rangle \in R^{n+1}$ and $\xi \in \Sigma$ implies that $\operatorname{Im} z \geqq \delta$, where $\Sigma=\left\{\xi \in R^{n}:|\xi|=1\right\}$ and $\xi^{\alpha}=\xi_{1}^{\alpha_{1}} \cdots \xi_{n}^{\alpha_{n}}$. We call $\delta$ a module of parabolicity for $P$. By means of a change of variables we can associate with $P$ the evolution operator $R=\partial / \partial t-H(t) \Lambda^{2 k}-J(t)$, where $H(t)$ and $J(t)$ are matrices of singular integral operators uniformly of order 0 and $2 k-1$, respectively on $R^{n}$.

In $\S \S 2$ and 3 we establish a generalization of the classical energy inequality for $R$ applicable to test functions on $R^{n+1}$. We prove this inequality by using the fact that the spectrum of the symbol of $H$ is contained in a compact subset of the open left-half complex plane (a trivial consequence of the parabolicity of $P$ ). We also apply certain estimates from [6] for pseudo-differential operators.

In $\$ \$ 4$ and 5 we first introduce the $\mathscr{H}^{r, s}$ spaces and the space $\mathscr{H}^{r, s}(\Omega)$ of restrictions to $\Omega$ of elements of $\mathscr{H}^{r, s}$, where $\Omega=\Omega_{a, b}=\left\{\langle x, t\rangle \in R^{n+1}: a<t<b\right\}$. Then by employing a form of the energy inequality applicable to distributions (Theorem 3 ), we deduce that

THEOREM 4. If $-\infty<a<b<+\infty$, if $r>(2 m-1) k$, and if $s$ is any real number, the mapping

$$
\phi \rightarrow \mathscr{P}_{\phi}=\left\langle P \phi, \phi(a),(\partial / \partial t) \phi(a),(\partial / \partial t)^{2} \phi(a), \ldots,(\partial / \partial t)^{m-1} \phi(a)\right\rangle
$$

is one-to-one from $\mathscr{H}^{\text {r,s }}(\Omega)$ into

$$
\mathscr{H}^{r-2 k m, s}(\Omega) \oplus H^{r+s-k} \oplus H^{r+s-3 k} \oplus \cdots \oplus H^{r+s-(2 m-1) k} .
$$

In $\S 6$ we show that $R+\lambda I$ is a topological isomorphism of $\left\{\mathscr{H}^{r, s}\right\}^{m}$ onto $\left\{\mathscr{H}^{r-2 k, s}\right\}^{m}$, provided $\lambda$ is sufficiently large (by $A^{m}$, where $A$ is a nonempty set, we mean $A \times A \times \cdots \times A, m$ times). To prove this result we employ our estimates from [6] and a commutator estimate from [5]. By employing the energy inequality once again we deduce that

THEOREM 7. If $-\infty<a<b<+\infty$, if $r>k$, and if $s$ is any real number the mapping $u \rightarrow \mathscr{R} u=\langle R u, u(a)\rangle$ is a topological isomorphism of $\left\{\mathscr{H}^{r, s}(\Omega)\right\}^{m}$ onto

$$
\left\{\mathscr{H}^{r-2 k, s}(\Omega)\right\}^{m} \oplus\left\{\boldsymbol{H}^{r+s-k}\right\}^{m} .
$$

In $\S 7$ we show that $\mathscr{P}: \mathscr{H}^{r, s}(\Omega) \rightarrow \mathscr{H}^{r-2 k m, s}(\Omega) \oplus H^{r+s-k} \oplus \cdots \oplus H^{r+s-(2 m-1) k}$ is an onto mapping for all $r>(2 m-1) k, r$ not an odd multiple of $k$ and $s$ any real number. We prove this by first showing that the inclusion mapping $i: \mathscr{H}^{r, s}(\Omega) \rightarrow \mathscr{H}^{r-2 k, s}(\Omega)$ has operator norm less than or equal to $C_{r}(b-a)$ (Theorem 9), where $r$ is not an odd multiple of $k, \Omega=\Omega_{a, b}$, and then applying Theorem 7 to the evolution operator corresponding to a certain truncation of $P$. 
2. The basic inequality.

Notation. For $\zeta, \eta \in C^{m}$ we use the usual notation $(\zeta, \eta)=\sum_{j=1}^{m} \zeta_{j} \bar{\eta}_{j}$ and $|\zeta|^{2}$ $\equiv(\zeta, \zeta)$. If $A=\left(a_{i j}\right)$ is a complex $m \times m$ matrix, the norm of $A$, denoted by $\|A\|$, is given by $\left\{\sum_{i, j}\left|a_{i j}\right|^{2}\right\}^{1 / 2}$.

Let $0<\delta<1$ be a module of parabolicity for $P$ which shall remain fixed throughout this paper. Let $\Delta$ be a compact subset of $C$ satisfying the property: $z \in \Delta \Rightarrow \operatorname{Re} z \leqq-\delta$. We define $\Pi(\Delta)$ to be the class of complex $m \times m$ matrices $h$ of the form

$$
h=i\left(\begin{array}{cccc}
0 & 1 & & 0 \\
0 & 0 & 1 & \\
& & & 1 \\
-p_{m} & -p_{m-1} & -\cdots & -p_{1}
\end{array}\right)
$$

such that the eigenvalues of $h$ are contained in $\Delta$.

THEOREM 1. Let $\delta$ and $\Pi(\Delta)$ be as above. Then there exists a constant $C_{0}>0$ (depending only on $\Delta, \delta$ and $m$ ) such that given any matrix $h \in \Pi(\Delta)$ there exists a nonsingular matrix $N(h)$ with the following properties:

(a) $\operatorname{Re}\left(R_{t}^{-1} N(h)^{-1} h N(h) R_{t} \zeta, \zeta\right) \leqq-(\delta / 4)|\zeta|^{2}$ for all $\zeta \in C^{m}$ and $t \in(0, \delta / 2]$, where

$$
R_{t}=\left|\begin{array}{cccccc}
1 & & & & \\
& t & & 0 & \\
& & t^{2} & & \\
& & \cdot & & \\
& & \cdot & \\
& 0 & & \cdot & \\
& & & & t^{m-1}
\end{array}\right| \text {, }
$$

and

(b) $\|N(h)\|+\left\|N(h)^{-1}\right\| \leqq C_{0}$.

LemMa 1. Suppose $m$ is a positive integer, and suppose $0<\theta<\frac{1}{2}$. Let the function $\tau$ be defined by $\tau(\rho)=\theta \rho^{M}$ where $M=m(m-1) / 2$. Then there exists $\varepsilon=\varepsilon(\theta, m)>0$ with the following properties: if $\Lambda$ is any set of complex numbers with no more than $m$ elements, then either

(a) there exists $\lambda_{1} \in \Lambda$ such that $\lambda \in \Lambda \Rightarrow\left|\lambda-\lambda_{1}\right|<\theta$, or

(b) there exists $\lambda_{1}, \lambda_{2}, \ldots, \lambda_{k} \in \Lambda(1<k \leqq m)$ such that

(i) $\rho=\min \left\{\left|\lambda_{i}-\lambda_{j}\right|: 1 \leqq i \leqq j\right\}>\varepsilon$, and

(ii) for every $\lambda \in \Lambda$, there exists $\lambda_{i}, 1 \leqq i \leqq k$, such that $\left|\lambda-\lambda_{i}\right|<\tau(\rho)$.

Proof. See $\S 8$ below.

Proof of Theorem 1. First we fix $\theta \in\left(0, \frac{1}{2}\right)$, and let $\varepsilon=\varepsilon(\theta, m)$ from Lemma 1 . Let $\Pi_{\varepsilon}(\Delta)$ be the collection of matrices $h$ in $\Pi(\Delta)$ having the property that $h$ has at least two distinct eigenvalues (thus $m \geqq 2$ ) and that the minimum distance 
between the distinct eigenvalues of $h$ is greater than $\varepsilon(\theta, m)$. Now let $h \in \Pi_{\varepsilon}(\Delta)$ and suppose $\lambda_{1}, \ldots, \lambda_{k}, 1<k \leqq m$, are the distinct eigenvalues of $h$ with multiplicities $\mu_{1}, \ldots, \mu_{k}$, respectively. Let $\rho=\rho(h)=\min \left\{\left|\lambda_{i}-\lambda_{j}\right|: 1 \leqq i<j<k\right\}>\varepsilon(\theta, m)$; we may assume $\rho<1$.

Let $N(h)$ be the $m \times m$ matrix

$$
N\left(\ell_{1}\right)=\left(e\left(\lambda_{1}\right) e^{\prime}\left(\lambda_{1}\right) \cdots \frac{e^{\left(\mu_{1}-1\right)}\left(\lambda_{1}\right)}{\left(\mu_{1}-1\right) !} \cdots e\left(\lambda_{k}\right) e^{\prime}\left(\lambda_{k}\right) \cdots \frac{e^{\left(\mu_{k}-1\right)}\left(\lambda_{k}\right)}{\left(\mu_{k}-1\right) !}\right)
$$

whose column vectors are defined starting from

$$
e(\lambda)=\left(\begin{array}{l}
1 \\
\lambda \\
\vdots \\
\lambda^{m-1}
\end{array}\right)
$$

and differentiating with respect to $\lambda \in \boldsymbol{C}$. Considering $N(h)$ as a linear transformation of $C^{m}$ into $C^{m}$ on the standard basis $\left\{\varepsilon_{1}, \varepsilon_{2}, \ldots, \varepsilon_{m}\right\}, \varepsilon_{j}$ has 1 as its $j$ th component and zeros elsewhere, we have that

$$
\begin{aligned}
& N(h) \varepsilon_{1}=e\left(\lambda_{1}\right), N(h) \varepsilon_{2}=e^{\prime}\left(\lambda_{1}\right), \ldots, \\
& N(h) \varepsilon_{\mu_{1}}=\left\{1 /\left(\mu_{1}-1\right) !\right\} e^{\left(\mu_{1}-1\right)}\left(\lambda_{1}\right), \ldots, N(h) \varepsilon_{m}=\left\{1 /\left(\mu_{k}-1\right) !\right\} e^{\left(\mu_{k}-1\right)}\left(\lambda_{k}\right) .
\end{aligned}
$$

Since $e(\lambda)$ and its derivatives are bounded on $\Delta$, there exists $C>0$ (depending only on $\Delta$ and $m$ ) such that $\|N(h)\| \leqq C$ for all $h \in \Pi(\Delta)$. Silov [8] shows that if $\tau_{1}, \tau_{2}, \ldots, \tau_{m}$ are $m$ arbitrary complex numbers having a subset of distinct points $\tau_{1}, \tau_{k}, \tau_{l}, \ldots, \tau_{p}$, where $1<k<l<\cdots<p \leqq m$, then

$$
\begin{aligned}
\operatorname{det}\left(e\left(\tau_{1}\right) e^{\prime}\left(\tau_{1}\right)\right. & \left.\cdots \frac{e^{(k-1)}\left(\tau_{1}\right)}{(k-1) !} \cdots e\left(\tau_{p}\right) e^{\prime}\left(\tau_{p}\right) \cdots \frac{e^{(m-p-1)}\left(\tau_{p}\right)}{(m-p-1) !}\right) \\
& =\lim \left(\frac{\prod_{1 \leqq i<j<k}\left(\tau_{j}-\tau_{i}\right) \prod_{k \leqq i_{k}<j_{k}<l}\left(\tau_{j_{k}}-\tau_{i_{k}}\right) \cdots \tau_{p \leqq i_{p}<j_{p} \leqq m}\left(\tau_{j_{p}}-\tau_{i_{p}}\right)}{\left.\prod_{j}-\tau_{i}\right)}\right)
\end{aligned}
$$

where the limit is taken as $\tau_{j} \rightarrow \tau_{1}, \tau_{j_{k}} \rightarrow \tau_{k}, \ldots, \tau_{j_{p}} \rightarrow \tau_{p}$. It is easily seen that the above limit is equal to a polynomial in $\tau_{1}, \tau_{k}, \ldots, \tau_{p}$ of the form

$$
\prod_{i, j=1, k, l, \ldots, p ; i<j}\left(\tau_{j}-\tau_{i}\right)^{\alpha_{i j}}
$$

where the $\alpha_{i j}$ 's are positive integers satisfying $\sum_{i j} \alpha_{i j} \leqq m(m-1) / 2=M$. Taking $\lambda_{1}=\tau_{1}, \lambda_{2}=\tau_{k}, \ldots, \lambda_{k}=\tau_{p}$ with the appropriate multiplicities $\mu_{1}, \mu_{2}, \ldots, \mu_{k}$ we see that $|\operatorname{det} N(h)| \geqq \rho(h)^{M}>\varepsilon(\theta, m)^{M}$ for all $h \in \Pi_{\varepsilon}(\Delta)$. Thus, there exists $C>0$ (depending on $\Delta$ and $m$ ) such that

$$
\left\|N(h)^{-1}\right\| \leqq C / \rho(h)^{M}<C / \varepsilon^{M}
$$


for all $h \in \Pi_{\varepsilon}(\Delta), \varepsilon=\varepsilon(\theta, m)$. The matrix form of $N(h)^{-1} \ell N(h)$ with respect to the basis $\left\{\varepsilon_{1}, \ldots, \varepsilon_{m}\right\}$ is easily determined by considering how $\ell$ acts on the basis

$$
B=\left(e\left(\lambda_{1}\right), e^{\prime}\left(\lambda_{1}\right), \ldots, \frac{e^{\left(\mu_{1}-1\right)}\left(\lambda_{1}\right)}{\left(\mu_{1}-1\right) !}, \ldots, e\left(\lambda_{k}\right), \ldots, \frac{e^{\left(\mu_{k}-1\right)}\left(\lambda_{k}\right)}{\left(\mu_{k}-1\right) !}\right)
$$

The characteristic polynomial for $h$ is given by $p(z)=z^{m}+\sum_{j=1}^{m} p_{j} z^{m-j}$, where the $p_{j}$ 's are the entries of $h$ in the form (2.1). Since

$$
h e(\lambda)=\lambda e(\lambda)-p(\lambda) \varepsilon_{m}
$$

we obtain, by differentiating with respect to $\lambda$,

$$
h \frac{e^{(j)}(\lambda)}{j !}=\lambda \frac{e^{(j)}(\lambda)}{j !}+\frac{e^{(j-1)}(\lambda)}{(j-1) !}-\frac{1}{j !} p^{(j)}(\lambda) \varepsilon_{m}
$$

for each $j=1,2, \ldots, m$. Since $\lambda_{l}$ is an eigenvalue of $h$ of multiplicity $\mu_{l}$, we have that $p\left(\lambda_{l}\right)=p^{(1)}\left(\lambda_{l}\right)=\cdots=p^{\left(\mu_{l}-1\right)}\left(\lambda_{l}\right)=0$. Thus

$$
\begin{gathered}
h e\left(\lambda_{l}\right)=\lambda_{l} e\left(\lambda_{l}\right), \quad \text { and } \\
h \frac{e^{(j)}\left(\lambda_{l}\right)}{j !}=\lambda_{l} \frac{e^{(j)}\left(\lambda_{l}\right)}{j !}+\frac{e^{(j-1)}\left(\lambda_{l}\right)}{(j-1) !}
\end{gathered}
$$

for each $j, 1 \leqq j \leqq \mu_{l}-1$ where $l=1,2, \ldots, k$. Thus, the matrix form of $N(h)^{-1} h N(h)$ is easily seen to be the Jordan form:

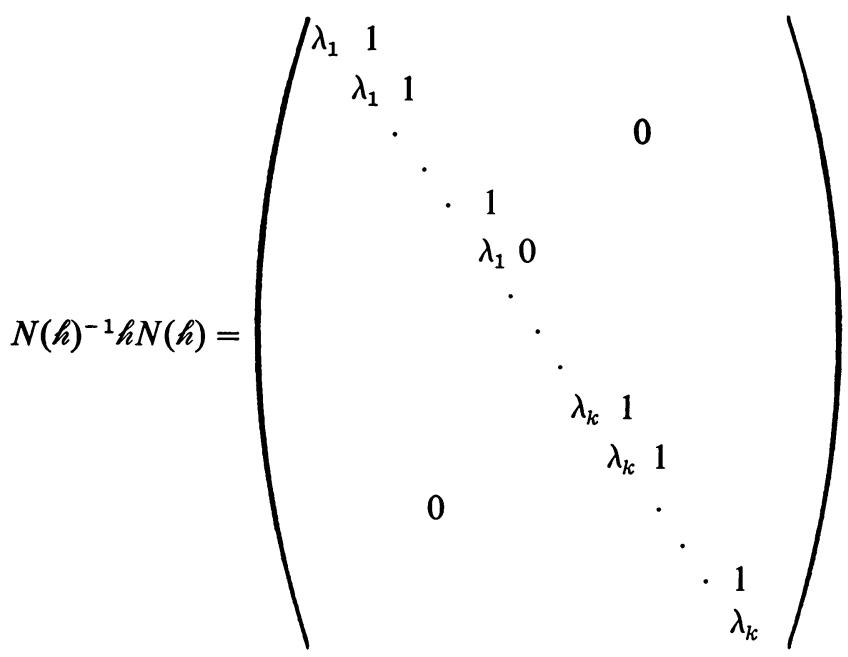

where each $\lambda_{l}$ appears $\mu_{l}$ times, $l=1,2, \ldots, k$.

We assert that

$$
\operatorname{Re}\left(R_{t}^{-1} N(h)^{-1} h N(h) R_{t} \zeta, \zeta\right) \leqq-(\delta / 2)|\zeta|^{2}
$$


for all $\zeta \in C^{m}$ and $t \leqq \delta / 2$. Since $R_{t}^{-1} N(h)^{-1} h N(h) R_{t}$ is a sum of matrices of the form:

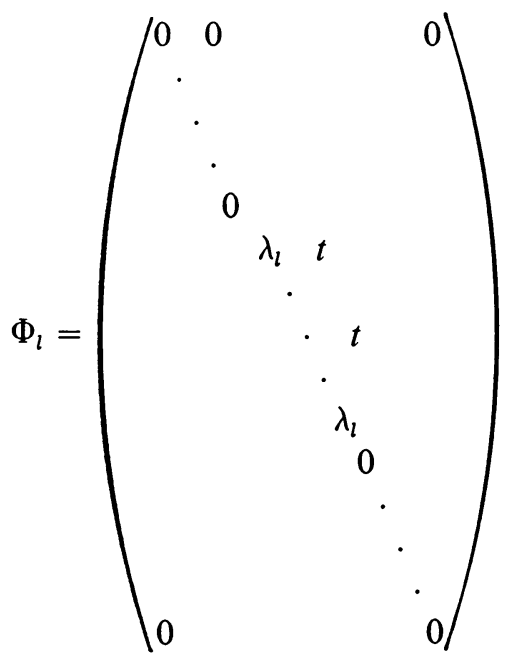

where $\lambda_{l}$ appears $\mu_{l}$ times, we shall first consider estimates on $\operatorname{Re}\left(\Phi_{l} \zeta, \zeta\right), 1 \leqq l \leqq k$. Without loss of generality we may assume $l=1$. Since $\lambda_{1} \in \Delta$ we have that

$$
\begin{aligned}
\operatorname{Re}\left(\Phi_{1} \zeta, \zeta\right) & =\operatorname{Re} \lambda_{1}\left(\left|\zeta_{1}\right|^{2}+\left|\zeta_{2}\right|^{2}+\cdots+\left|\zeta_{\mu_{1}}\right|^{2}\right)+t \operatorname{Re}\left(\zeta_{2} \zeta_{1}+\zeta_{3} \zeta_{2}+\cdots+\zeta_{\mu_{1}} \zeta_{\mu_{1}-1}\right) \\
& \leqq-\delta\left(\left|\zeta_{1}\right|^{2}+\cdots+\left|\zeta_{\mu_{1}}\right|^{2}\right)+t \operatorname{Re}\left(\zeta_{2} \zeta_{1}+\zeta_{3} \zeta_{2}+\cdots+\zeta_{\mu_{1}} \zeta_{\mu_{1}-1}\right)
\end{aligned}
$$

Since $\left\{\lambda_{1}, \lambda_{2}, \ldots, \lambda_{m}\right\} \subseteq \Delta$, we obtain

$$
\operatorname{Re}\left(R_{t}^{-1} N(h)^{-1} h N(h) R_{t} \zeta, \zeta\right)=\sum_{l=1}^{k} \operatorname{Re}\left(\Phi_{l} \zeta, \zeta\right)
$$

for all $\zeta \in C^{m}$. Thus (2.6) holds for all $h \in \Pi_{\varepsilon}(\Delta), \varepsilon=\varepsilon(\theta, m)$. We remark that the estimate (2.6) is independent of $\theta$.

We now wish to treat those matrices $h \in \Pi(\Delta)$ having the property that the minimum distance between the distinct eigenvalues of $h$ is less than $\varepsilon(\theta, m)$ (see Lemma 1). It is easy to see that $N(h)$, as constructed above, will not satisfy statement (b) of our theorem since, in general, $\left\|N(h)^{-1}\right\| \rightarrow \infty$ as the minimum distance between the distinct eigenvalues of $h$ approaches 0 . By taking $\theta$ sufficiently small (depending on $\Delta, \delta$ and $m$ ) we shall show that for each $h \in \Pi(\Delta)$ we can find a matrix $h \in \Pi_{\varepsilon(\theta, m)}(\Delta)$ satisfying the following:

(1) the eigenvalues of $h$ are "close" to the eigenvalues of $h$ by a distance less than $\varepsilon(\theta, m)$;

(2) the choice of $N(h)$, instead of $N(h)$, satisfies statement (a) of our theorem. $N(h)$ satisfies statement (b) of our theorem by our previous argument for matrices $h \in \Pi_{\varepsilon}(\Delta)$, where $\varepsilon=\varepsilon(\theta, m)$.

Let $h \in \Pi(\Delta)$ and let $\Lambda$ be its set of eigenvalues (counting multiplicities). By Lemma 1 there exist two possible configurations for the set $\Lambda$ : 
Case 1. There exists $\lambda_{1} \in \Lambda$ such that $\left|\lambda-\lambda_{1}\right|<\theta$ for all $\lambda \in \Lambda$, or

Case 2. There exists $\lambda_{1}, \lambda_{2}, \ldots, \lambda_{k} \in \Lambda(1<k \leqq m)$ such that

(i) $\rho=\min \left\{\left|\lambda_{i}-\lambda_{j}\right|: 1 \leqq i<j \leqq k\right\}>\varepsilon=\varepsilon(\theta, m)$,

(ii) for every $\lambda \in \Lambda$, there exists $\lambda_{i}, 1 \leqq i \leqq k$, such that $\left|\lambda-\lambda_{i}\right|<\tau(\rho) \equiv \theta \rho^{M}$, where $M=m(m-1) / 2$.

Let $\Pi_{\varepsilon}^{1}(\Delta)$ and $\Pi_{\varepsilon}^{2}(\Delta)$ be the sets of matrices $h$ in $\Pi(\Delta)$ whose eigenvalues satisfy Case 1 and Case 2, respectively. Thus $\Pi(\Delta)=\Pi_{\varepsilon}^{1}(\Delta) \cup \Pi_{\varepsilon}^{2}(\Delta)$. Let $h \in \Pi_{\varepsilon}^{2}(\Delta)$ and suppose $h$ has eigenvalues $\lambda_{1}, \ldots, \lambda_{m}$ (note that $m>1$ ). If $k=m$, we have, by our previous argument, that the matrix $N(h)=\left(e\left(\lambda_{1}\right) \cdots e\left(\lambda_{m}\right)\right)$ satisfies (2.6) with $h=h$ and that $\left\|N(h)^{-1}\right\|<C / \varepsilon^{M}$, where $\varepsilon=\varepsilon(\theta, m)$ and $C>0$ depends only on $\Delta, \delta$ and $m$. Now if $k<m$, then $\rho_{h}=\min \left\{\left|\lambda_{i}-\lambda_{j}\right|: 1 \leqq i \leqq j \leqq k\right\}>\varepsilon(\theta, m)$. Let us consider open disks $S_{1}, S_{2}, \ldots, S_{k}$ of radius $\tau\left(\rho_{h}\right)$ centered at the points $\lambda_{1}, \lambda_{2}, \ldots, \lambda_{k}$, respectively. By Case 2(ii), each of the points $\lambda_{k+1}, \ldots, \lambda_{m}$ is contained in one of these disks; moreover, since $\theta<\frac{1}{2}$, each of the points $\lambda_{k+1}, \ldots, \lambda_{m}$ is contained in only one of these disks. Assuming that for each $l, 1 \leqq l \leqq k, S_{l}$ contains exactly $\mu_{l}$ eigenvalues of $h$, we have that $\sum_{l=1}^{k} \mu_{l}=m$. Define the complex numbers $\tilde{p}_{1}, \ldots, \tilde{p}_{m}$ by

$$
\tilde{p}(z) \equiv \prod_{l=1}^{k}\left(z-\lambda_{l}\right)^{\mu_{l}}=z^{m}+\sum_{j=1}^{m} \tilde{p}_{j} z^{m-j} .
$$

If we define $h$ by

$$
h=i\left(\begin{array}{ccccc}
0 & 1 & & & 0 \\
0 & 0 & 1 & & \\
& & & \cdot & \\
& & & \cdot & \\
0 & 0 & & 1 \\
-\tilde{p}_{m} & -\tilde{p}_{m-1} & & \ldots & -\tilde{p}_{1}
\end{array} \mid\right.
$$

then $h \in \Pi_{\varepsilon}(\Delta)$ by our previous argument, and we say that $h$ is a perturbation of $h$. We remark that $N(h)$ is only defined for $h \in \Pi_{\varepsilon}(\Delta)$. Thus $N(h)$ is given by

$$
N(h)=\left(e\left(\lambda_{1}\right) e^{\prime}\left(\lambda_{1}\right) \cdots \frac{e^{\left(\mu_{1}-1\right)}\left(\lambda_{1}\right)}{\left(\mu_{1}-1\right) !} \cdots e\left(\lambda_{k}\right) e^{\prime}\left(\lambda_{k}\right) \cdots \frac{e^{\left(\mu_{k}-1\right)}\left(\lambda_{k}\right)}{\left(\mu_{k}-1\right) !}\right) .
$$

Considering $N(h)$ as a linear transformation of $C^{m}$ into itself on the basis $\left\{\varepsilon_{1}, \ldots, \varepsilon_{m}\right\}$, we know by our previous arguments that there exists $C>0$ (depending on $\Delta, m$ and $\delta$ ) such that $\|N(h)\| \leqq C$ and $\left\|N(h)^{-1}\right\|<C\left(\varepsilon(\theta, m)^{-M}\right)$ for $h \in \Pi_{\varepsilon}(\Delta)$. Returning to $h$ given by (2.7) and its perturbation $h$ we know that the matrix form of $N(h)^{-1} h N(h)$ with respect to $\left\{\varepsilon_{1}, \ldots, \varepsilon_{m}\right\}$ is determined by its action on the basis $B$. Letting $p(z) \equiv z^{m}+\sum_{j=1}^{m} p_{j} z^{m-j}$, where the $p_{j}$ 's are the entries of $h$ in the form (2.1), we have that $h e(\lambda)=\lambda e(\lambda)-p(\lambda) \varepsilon_{m}$, and

$$
h \frac{e^{(j)}(\lambda)}{j !}=\lambda \frac{e^{(j)}(\lambda)}{j !}+\frac{e^{(j-1)}(\lambda)}{(j-1) !}-\frac{1}{j !} p^{(j)}(\lambda) \varepsilon_{m}
$$


for each $j=1,2, \ldots, m$. Thus, by definition of $N(h)$, we obtain $h e\left(\lambda_{l}\right)=\lambda_{l} e\left(\lambda_{l}\right)$, and

$$
h \frac{e^{(j)}\left(\lambda_{l}\right)}{j !}=\lambda_{l} \frac{e^{(j)}\left(\lambda_{l}\right)}{j !}+\frac{e^{(j-1)}\left(\lambda_{l}\right)}{(j-1) !}-\frac{1}{j !} p^{(j)}\left(\lambda_{l}\right) N(h)^{-1}\left(\frac{e^{\left(\mu_{k}-1\right)}\left(\lambda_{k}\right)}{\left(\mu_{k}-1\right) !}\right), \quad 1 \leqq j \leqq \mu_{l}-1,
$$

for each $l=1,2, \ldots, k$. Thus the matrix form of $h$ in the basis $B$ is

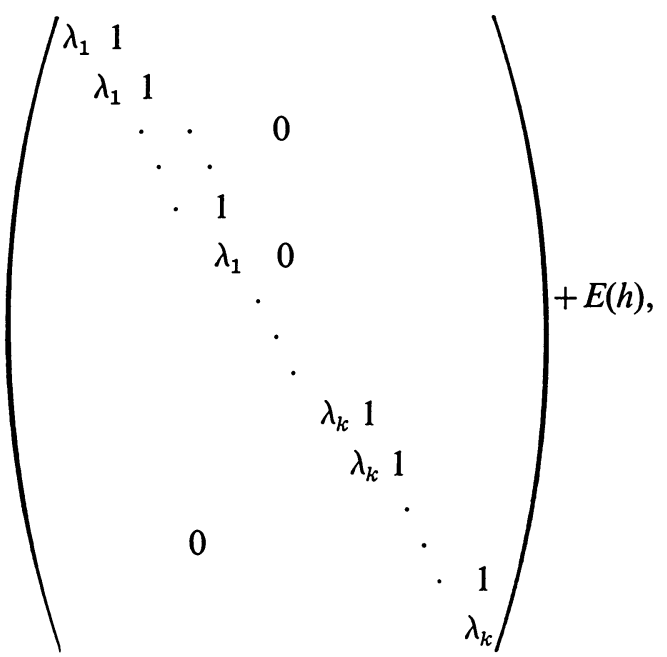

where $\lambda_{l}$ appears $\mu_{l}$ times, $l=1, \ldots, k$, and the column vectors of the "error" matrix $E(h)$ are either 0 or of the form $\left(p^{(j)}\left(\lambda_{l}\right) / j !\right) N(h)^{-1}\left(e^{\left(\mu_{k}-1\right)}\left(\lambda_{k}\right) /\left(\mu_{k}-1\right) !\right)$, $1 \leqq j \leqq \mu_{l}-1, l=1,2, \ldots, k, \mu_{l}>1$. If $h \in \Pi_{\varepsilon}(\Delta)$, we have, by our previous argument, $E(h)=0$. Since $N(h)^{-1} h N(h)$ has the form (2.8), by (2.6) we obtain

$$
\operatorname{Re}\left(R_{t}^{-1} N(h)^{-1} h N(h) R_{t} \zeta, \zeta\right) \leqq\{\|E(h)\|-\delta / 2\}|\zeta|^{2}
$$

for all $\zeta \in C^{m}$ and $t \leqq \delta / 2$. Since $e^{(\mu)}(\lambda) / \mu$ is bounded for $\lambda \in \Delta$ we have that $\|E(h)\|$ is bounded by a finite number of terms of the form $C\left\|N(h)^{-1}\right\|\left\{(1 / j !)\left|p^{(j)}\left(\lambda_{l}\right)\right|\right\}$, where $1 \leqq j \leqq \mu_{l}-1, \mu_{l}>1$ and $l=1,2, \ldots, k$. In general, for $1 \leqq l \leqq k$, we know that

$$
\frac{p^{(\nu)}\left(\lambda_{l}\right)}{\nu !}=C_{\nu} \sum_{k_{1}, k_{2}, \ldots, k_{\nu}-1}^{k} \prod_{\substack{1 \leq j \leq k, j \neq l, j \neq k_{1}, \ldots, k_{\nu}-1}}\left(\lambda_{l}-\lambda_{j}\right), \quad 1 \leqq \nu \leqq m .
$$

Since each of the points $\lambda_{k+1}, \ldots, \lambda_{m}$ is contained in exactly one of the disks $S_{1}, S_{2}, \ldots, S_{k}$, each having radius $\tau\left(\rho_{h}\right)$, we obtain

$$
(1 / j !)\left|p^{(j)}\left(\lambda_{l}\right)\right| \leqq C_{m} \tau\left(\rho_{h}\right)^{u_{j}-j} \leqq C_{m} \tau\left(\rho_{h}\right)
$$

for $1 \leqq j \leqq \mu_{l}-1, \mu_{l}>1$ and $l=1,2, \ldots, k$. Since $\rho_{h}=\rho(h)$ we combine the above estimate with the estimate $\left\|N(h)^{-1}\right\| \leqq C_{1} \rho(h)^{-M}$ to obtain $\|E(h)\| \leqq C_{1} \rho(h)^{-M}$ - $\theta \rho(h)^{M}=C_{1} \theta$ where $C_{1}>0$ depends only on $\Delta, \delta$ and $m$. Taking $\theta$ such that

$$
0<\theta<\min \left\{\frac{1}{2}, \delta / 4 C_{1}\right\}
$$

we see that (2.9) yields

$$
\operatorname{Re}\left(R_{t}^{-1} N(h)^{-1} h N(h) R_{t} \zeta, \zeta\right) \leqq-(\delta / 4)|\zeta|^{2}
$$


for all $\zeta \in C^{m}$ and $t \leqq \delta / 2$. Letting $N(h)=N(h)$ our theorem is proved for all $h \in \Pi_{\varepsilon}^{2}(\Delta)$ where $\varepsilon=\varepsilon(\theta, m)$ with $\theta$ fixed and satisfying (2.10).

Now suppose $h \in \Pi_{\varepsilon}^{1}(\Delta)$ with eigenvalues $\left\{\lambda_{1}, \ldots, \lambda_{m}\right\}$. By renumbering we may assume that $\left|\lambda_{i}-\lambda_{1}\right|<\theta$ for each $i=2, \ldots, m$; thus $k=1$. Since

$$
N(h)=\left(e\left(\lambda_{1}\right) e^{\prime}\left(\lambda_{1}\right) \cdots \frac{e^{(m-1)}\left(\lambda_{1}\right)}{(m-1) !}\right)
$$

it is easily seen that there exists $C>0$ (depending on $\Delta, \delta$ and $m$ ) such that $\|N(h)\|$ $\leqq C$ for all $h \in \Pi_{\varepsilon}^{1}(\Delta), \varepsilon=\varepsilon(\theta, m)$. Since $|\operatorname{det} N(h)|=1$ we have that $\left\|N(h)^{-1}\right\| \leqq C$ for all $h \in \Pi_{\varepsilon}^{1}(\Delta)$, where $C>0$ depends only on $\Delta, \delta$ and $m$. Expressing $N(h)^{-1} h N(h)$ in the form (2.8) we see that the "error" term $\|E(h)\|$ is easily estimated by $C_{2} \theta$, where $C_{2}$ depends only on $\Delta, \delta$ and $m$. Taking $\theta$ such that $0<\theta<\min \left\{\frac{1}{2}, \delta / 4 C_{1}, \delta / 4 C_{2}\right\}$ our proof is complete.

\section{The energy inequality for test functions.}

The $H^{s}$ spaces on $R^{n}$. (i) For $\phi \in \mathscr{D}\left(R^{n}\right) \equiv C_{0}^{\infty}\left(R^{n}\right)$, the space of infinitely differentiable functions with compact support, $\tilde{\phi}$ denotes the $n$-dimensional Fourier transform of $\phi$ :

$$
\delta(\xi)=(2 \pi)^{-n / 2} \int_{R^{n}} e^{-i\langle\xi, x\rangle} \phi(x) d x
$$

(here $\left.\langle\xi, x\rangle=\sum_{j=1}^{n} \xi_{j} x_{j}\right) . \mathscr{S}\left(R^{k}\right)$ denotes the space of infinitely differentiable and rapidly decreasing functions on $R^{k} ; \mathscr{S}^{\prime}\left(R^{k}\right)$ denotes the space of tempered distributions on $R^{k}$. For real $s$ we define

$$
H^{s}=\left\{u \in \mathscr{S}^{\prime}\left(R^{n}\right): \tilde{u} \in L_{\mathrm{loc}}^{1}\left(R^{n}\right) \text { and } \int_{R^{n}}\left\{1+|\xi|^{2}\right\}^{s}|\tilde{u}(\xi)|^{2} d \xi \equiv\|u\|_{s}^{2}<\infty\right\} .
$$

$H^{s}$ is a Hilbert space with the scalar product $(,)_{s}$ defined in the obvious way; here, we use the usual extension to $\mathscr{S}^{\prime}\left(R^{n}\right)$ of $u m \sim \tilde{u}$ on $R^{n}$.

(ii) The topological inclusions $\mathscr{D}\left(R^{n}\right) \subset \mathscr{S}\left(R^{n}\right) \subset H^{s}$ are dense.

(iii) For $\phi, \psi \in \mathscr{D}\left(R^{n}\right)$ we write $(\phi, \psi)_{0}=\int_{R^{n}} \phi(x) \bar{\psi}(x) d x$. Also for $\phi \in \mathscr{D}\left(R^{n}\right)$ and any $s$,

$$
\|\phi\|_{s}=\sup \left\{\left|(\phi, \psi)_{0}\right| /\|\psi\|_{-s}: \psi \in \mathscr{D}\left(R^{n}\right), \psi \neq 0\right\} .
$$

Thus $H^{s}$ and $H^{-s}$ are dual Hilbert spaces, the duality being given by the sesquilinear form (which we again denote by $(,)_{0}$ ) obtained by extension.

(iv) If $s<t$ then $H^{t} \subset H^{s}$ and $\|u\|_{s}<\|u\|_{t}$. Given $s_{1} \leqq t<s_{2}$ and any $\varepsilon>0$, there exists $C(\varepsilon)>0$ such that

$$
\|u\|_{t}^{2} \leqq \varepsilon\|u\|_{s_{2}}^{2}+C(\varepsilon)\|u\|_{s_{1}}^{2}
$$

for all $u \in H^{s_{2}}$. Note that $H^{\infty} \equiv \bigcap_{s} H^{s} \subset C^{\infty}$.

(v) Let $L$ be a linear operator from $\mathscr{S}\left(R^{n}\right)$ into $H^{\infty}$. Following Kohn and Nirenberg [6], we say that $L$ has order $r$ if for each real number $s$ there exists a constant $C_{s}>0$ such that $\|L u\|_{s} \leqq C_{s}\|u\|_{s+r}$ for all $u \in \mathscr{S}\left(R^{n}\right)$. The infimum of all orders of $L$ is called the true order of $L$. Let $L$ be an operator of order $r$. Then $L$ 
can be extended to a bounded operator $L_{s}$ from $H^{s}$ into $H^{s-r}$ for any real $s$. Since $L_{s}=L_{t}$ on $H^{s} \cap H^{t}$ for all $s$ and $t$, we shall denote all these extensions of $L$ by $L$. We also denote the extension of $L$ to an operator on $H^{s}$ by $L$. If $L$ and $L^{*}$ are linear maps of $\mathscr{S}\left(R^{n}\right)$ into $H^{\infty}$, and $(L \phi, \psi)_{0}=\left(\phi, L^{*} \psi\right)_{0}$ for all $\phi, \psi \in \mathscr{S}\left(R^{n}\right)$, then $L^{*}$ is called the formal adjoint of $L$. Since $\mathscr{S}\left(R^{n}\right)$ is dense in $\bigcup_{s} H^{s}, L^{*}$ is unique. If $L$ is of order $r$, then its extension $L_{s}$ has an adjoint $L_{s}^{*}$ which is bounded from $H^{-s+r}$ into $H^{-s}$. Thus $L$ has a formal adjoint $L^{*}$ of order $s$ whose restriction to $\mathscr{S}\left(R^{n}\right)$ is $L_{s}^{*}$. If $L$ and $M$ are operators of order $r$ and $s$, respectively, then the composite operator $L M$ has order $r+s$.

If $a(\xi)$ is a complex valued measurable function on $R^{n} \sim\{0\}$ such that, for some number $r$ and $C>0,|a(\xi)| \leqq C\left\{1+|\xi|^{2}\right\}^{r / 2}$ for all $\xi \in R^{n} \sim\{0\}$, then the operator $a(D)$ defined by $\mathscr{F}(a(D) u)(\xi)=a(\xi) \tilde{u}(\xi)$ for $u \in \mathscr{S}\left(R^{n}\right)$ is of order $r$. In particular we define the operators:

and

$$
\Lambda^{s}=\lambda_{s}(D), \quad \text { where } \lambda_{s}(\xi)=|\xi|^{s}, \text { where } s \geqq 0,
$$

$$
R^{\alpha}=r^{\alpha}(D), \quad \text { where } r^{\alpha}(\xi)=(\xi /|\xi|)^{\alpha} .
$$

The operators $\Lambda^{s}$ and $R^{\alpha}$ have orders $s$ and zero, respectively, and $D^{\alpha}=\Lambda^{|\alpha|} R^{\alpha}$. Operators of the form $a(D)$ are called multipliers.

(3.3) Definition. We say $a(x, \xi) \in C^{\infty}\left(R^{n} \times R^{n} \sim\{0\}\right)$ is a symbol on $R^{n} \times \Sigma$ if

(i) $a(x, \xi)$ is homogeneous in $\xi$ of degree zero, and

(ii) for each multi-index $\alpha$ there exists $C_{\alpha}>0$ such that $\left|D_{x}^{\alpha} a(x, \xi)\right| \leqq C_{\alpha}$ for all $\langle x, \xi\rangle \in R^{n} \times \Sigma$. (Note that $a(x, \xi)$ is not a symbol in the sense of Kohn and Nirenberg [6], since we do not insist that $a(x, \xi)$ converge rapidly to a unique limit $a(\infty, \xi)$ as $x \rightarrow \infty$.) We associate with the symbol $a(x, \xi)$ the formal operator $A=a(x, D)$ defined by

$$
A u(x)=(2 \pi)^{-n / 2} \int_{R^{n}} e^{i\langle x, \xi\rangle} a(x, \xi) \tilde{u}(\xi) d \xi, \quad u \in \mathscr{S}\left(R^{n}\right) .
$$

We say $A$ is a singular integral operator on $R^{n}$ with symbol $a(x, \xi) . A$ is of order zero (see [7]). If $a(x, \xi) \equiv a(\xi)$ then $a(x, D)$ is easily seen to be the multiplier $a(D)$. If $a(x, \xi) \equiv a(x)$ (thus $a \in C_{B}^{\infty}\left(R^{n}\right)$ ), then $a(x, D)$ is the operator multiplication by $a$ which we denote by $a \cdot$.

Let $a(x, \xi)$ be a symbol on $R^{n} \times \Sigma$. Then, for $k, p$ nonnegative integers, we define

$$
\|a\|_{[k, p]}=\sum_{|\alpha| \leqq k} \sup _{|\beta| \leqq p}\left\{\left|D_{x}^{\alpha} \partial_{\xi}^{\beta} a(x, \xi)\right|: x \in R^{n}, \xi \in \Sigma\right\} .
$$

We define the pseudo-adjoint of $A=a(x, D)$, denoted by $A^{\#}$, as the singular integral operator with symbol $\overline{a(x, \xi)}$. If $B=b(x, D)$ is another singular integral operator, we define the pseudo-product of $A$ and $B$, denoted by $A \circ B$, as the singular integral operator with symbol $a(x, \xi) b(x, \xi)$ (thus $A \circ B=B \circ A)$.

Proposition 1. Let $A=a(x, D)$ and $B=b(x, D)$ be singular integral operators. Then for every integer $m$ there is a constant $C_{m}>0$ such that 
(i) $A$ is of order zero and

$$
\|A u\|_{m} \leqq C_{m}\|a\|_{[|m|+1, n+1]}\|u\|_{m},
$$

(ii) $A^{*}-A^{\#}$ is of order -1 and

$$
\left\|\left(A^{*}-A^{\#)}\right) u\right\|_{m+1} \leqq C_{m}\|a\|_{[|m|+2,2 n+3]}\|u\|_{m},
$$

(iii) $A B-A \circ B$ is of order -1 and

$$
\|(A B-A \circ B) u\|_{m+1} \leqq C_{m}\|a\|_{[|m|+2,2 n+3]}\|b\|_{[|m|+2,2 n+3]}\|u\|_{m}
$$

for all $u \in H^{m}$.

Proof. See $\$ 5,6$ and 7 of [7].

COROLlARY. Let $A$ and $B$ be singular integral operators as above. Then for every $s$ there is a constant $C_{s}>0$ such that

$$
\begin{aligned}
\|A u\|_{s} & \leqq C_{s}\|a\|_{[|m|+1, n+1]}\|u\|_{s}, \\
\left\|\left(A^{*}-A^{\#}\right) u\right\|_{s+1} & \leqq C_{s}\|a\|_{[|m|+3,2 n+3]}\|u\|_{s}, \\
\|(A B-A \circ B) u\|_{s+1} & \leqq C_{s}\|a\|_{[|m|+3,2 n+3]}\|b\|_{[|m|+3,2 n+3]}\|u\|_{s}
\end{aligned}
$$

for all $u \in H^{s}$, where $m=[s]$ (the integral part of $s$ ).

Proof. The proof is an easy consequence of Proposition 1 and an application of Calderón's multilinear interpolation theorem [2].

Let $k(x, t ; \xi),\langle x, t ; \xi\rangle \in R^{n+1} \times R^{n} \sim\{0\}$ satisfy

(i) $k(x, t ; \xi)$ is homogeneous in $\xi$ of degree zero, and

(ii) for each multi-index $\alpha$ there exists $C_{\alpha}>0$ such that

(ii) $\left|D_{x}^{\alpha} k(x, t ; \xi)\right| \leqq C_{\alpha}$ for all $\langle x, t ; \xi\rangle \in R^{n+1} \times \Sigma$.

Then, by the corollary to Proposition 1 , for $l, p$ nonnegative integers there exists $C=C(l, p)>0$ such that

$$
\|k(t)\|_{[l, p]} \leqq C \text { for all } t \in R^{1},
$$

where $k(t)$ is the function on $R^{n} \times R^{n} \sim\{0\}$ given by $\langle x, \xi\rangle \leadsto k(x, t ; \xi)$. Let $k(x, t ; \xi)$ satisfy condition (3.4) above. For each $t \in R^{1}$ we define $K(t)$ to be singular integral operator $k(x, t ; D)$. Thus, by Proposition $1(\mathrm{i}),\|K(t) u\|_{s} \leqq C_{s}\|u\|_{s}$, for all $u \in H^{s}$ and all $t \in R^{1}, s$ real.

Let $\phi(x, t)$ be a complex valued function on $R^{n+1}$; by $\phi(t)$ we mean the function on $R^{n}$ given by $x \sim \phi(x, t)$.

Reduction of $P$ to first order in $t$. We call

$$
P_{0}\left(x, t ; D, D_{t}\right)=\sum_{|\alpha|+2 k j=2 k m} a_{\alpha, j}(x, t) D^{\alpha} D_{t}^{j}
$$

the principal part of $P$. Since $a_{0, m} \equiv 1$ we can express $P$ in the form $P=P_{0}+P_{1}+Q$ where

$$
P_{0}(x, t ; \xi, \tau)=\tau^{m}+\sum_{j=1}^{m} p_{j}(x, t ; \xi) \tau^{m-j}
$$


with

$$
\begin{aligned}
p_{j}(x, t ; \xi) & =\sum_{|\alpha|=2 k j} a_{\alpha, m-j}(x, t) \xi^{\alpha}, \\
P_{1}(x, t ; \xi, \tau) & =\sum_{2 k(m-1) \leqq|\alpha|+2 k j<2 k m} a_{\alpha, j}(x, t) \xi^{\alpha} \tau^{j} \\
& =\sum_{j=1}^{m} \sum_{2 k(m-j) \leqq|\alpha|<2 k(m-j+1)} a_{\alpha, j-1}(x, t) \xi^{\alpha} \tau^{j-1}, \\
Q(x, t ; \xi, \tau) & =\sum_{|\alpha|+2 k j<2 k(m-1)} a_{\alpha, j}(x, t) \xi^{\alpha} \tau^{j} \\
& =\sum_{j=1}^{m-1} \sum_{|\alpha|<2 k(m-j)} a_{\alpha, j-1}(x, t) \xi^{\alpha} \tau^{j-1}
\end{aligned}
$$

(here we assume $m>1$, if $m=1$ we define $P_{1}=Q=0$ ).

We use a method of Calderón (see [3]) to reduce the study of $P$ to the study of an evolution operator $\partial / \partial t-H(t) \Lambda^{2 k}-J(t)$, where $H(t)$ and $J(t)$ are matrices of singular integral operators depending on $t \in R^{1}$. Let $\phi \in C_{0}^{\infty}\left(R^{n+1}\right)$ and let $u$ be the vector of functions $u=\left\langle u_{1}, u_{2}, \ldots, u_{m}\right\rangle$ where

$$
u_{j}=\Lambda^{2 k(m-j)} D_{t}^{j-1} \phi \text { for } j=1,2, \ldots, m \text {. }
$$

We have that $D_{t} u_{j}=\Lambda^{2 k} u_{j+1}$ for $j=1,2, \ldots, m-1$, and

$$
\begin{aligned}
D_{t} u_{m}= & D_{t}^{m} \phi \\
= & \left(P_{0}+P_{1}\right) \phi-\sum_{j=1}^{m} p_{j}(x, t ; D) D_{t}^{m-j} \phi \\
& -\sum_{j=1}^{m} 2 k(m-j) \leqq|\alpha|<2 k(m-j+1) \\
= & \left(P_{0}+P_{1}\right) \phi-\sum_{j=1}^{m} P_{j}(t) \Lambda^{2 k} u_{m-j+1}(x, t) D^{\alpha} D_{t}^{j-1} \phi \\
& -\sum_{j=1}^{m}\left\{\sum_{2 k(m-j) \leqq|\alpha|<2 k(m-j+1)} A_{\alpha, j-1}(t) \Lambda^{|\alpha|-2 k(m-j)}\right\} u_{j}
\end{aligned}
$$

where $P_{j}(t)$ and $A_{\alpha, j}(t)$ are singular integral operators on $R^{n}$ whose symbols are $p_{j}(x, t ; \xi /|\xi|)$ and $a_{\alpha, j}(x, t)(\xi /|\xi|)^{\alpha}$, respectively. Thus we have that

$$
\frac{\partial u}{\partial t}=H(t) \Lambda^{2 k} u+J(t) u+i\left(\left(P_{0}+P_{1}\right) \phi\right) \varepsilon_{m},
$$

where

$$
\varepsilon_{m}=\left(\begin{array}{c}
0 \\
0 \\
\vdots \\
0 \\
1
\end{array}\right)
$$

and the $l$ th component of $J(t) u$ is given by 


$$
\begin{aligned}
(J(t) u)_{l} & =0 \quad \text { if } l \neq m, \\
& =-i \sum_{j=1}^{m}\left\{\sum_{2 k(m-j) \leqq|\alpha|<2 k(m-j+1)} A_{\alpha, j-1}(t) \Lambda^{|\alpha|-2 k(m-j)}\right\} u_{j} \quad \text { if } l=m,
\end{aligned}
$$

and $H(t)$ is the matrix of singular integral operators whose symbol $h(t)$ is given by

$$
h(x, t ; \xi)=i\left(\begin{array}{rrrc}
0 & 1 & & 0 \\
0 & 0 & 1 & \\
0 & 0 & & \\
-p_{m}(x, t ; \xi|| \xi \mid) & -\cdots & -p_{1}(x, t ; \xi|| \xi \mid)
\end{array}\right)
$$

for $\langle x, t\rangle \in R^{n+1}$ and $\xi \in R^{n} \sim\{0\}$. We define the order of a matrix of operators in the obvious way. Since the coefficients of $P$ are elements of $C_{B}^{\infty}\left(R^{n+1}\right)$, we have, by (3.5), that $H(t)$ and $J(t)$ are uniformly of order zero and $2 k-1$ respectively, for $t \in R^{1}$.

By definition of $\delta$, a module of parabolicity for $P$ (which we have fixed in $\S 2$ ), we know that the zeros of $P_{0}(x, t ; \xi, z)$ in $z$ are contained in a compact subset of $\{z \in C: \operatorname{Im} z \geqq \delta\}$ independent of $\langle x, t ; \xi\rangle \in R^{n+1} \times \Sigma$. Also we observe that the eigenvalues of $-i \hbar(x, t ; \xi)$ are precisely the zeros of $P_{0}(x, t ; \xi, z)$ in $z$. Thus, there exists a compact subset of $C$, call it $\Delta$, having the property that $\Delta \subset\{z: \operatorname{Re} z \leqq-\delta\}$, and that for $\langle x, t ; \xi\rangle \in R^{n+1} \times \Sigma$ the eigenvalues of $h(x, t ; \xi)$ are contained in $\Delta$. $\Delta$ shall remain fixed throughout the remainder of this paper. If we let

$$
R=\partial / \partial t-H(t) \Lambda^{2 k}-J(t)
$$

we have that $R u=i\left[\left(P_{0}+P_{1}\right) \phi\right] \varepsilon_{m}=i[(P \phi-Q \phi)] \varepsilon_{m}$. This fact will be used in $\S 5$ where we will take $\phi$ to be a distribution on $R^{n+1}$. In this section we shall confine our study to the operator $R$.

Suppose $R$ is of the form

$$
R(h)=\partial / \partial t-h \Lambda^{2 k}-J(t),
$$

where $J(t)$ is given by (3.7) and $h$ is a constant matrix of the form (2.1) having all its eigenvalues contained in $\Delta$. If we let $t=\delta / 2$ and denote $N(h) R_{\delta / 2}$ by simply $N(h)$ in Theorem 1, then we can find constants $C_{i}=C_{i}(\Delta, \delta, m)>0, i=1,2$, such that for each $h \in \Pi(\Delta)$ there exists a nonsingular matrix $N(h)$ satisfying

$$
\begin{gathered}
C_{2}|N(h) \zeta| \leqq|\zeta| \leqq C_{1}|N(h) \zeta|, \\
\operatorname{Re}\left(N(h)^{-1} h N(h) \zeta, \zeta\right) \leqq-(\delta / 4)|\zeta|^{2}
\end{gathered}
$$

for all $\zeta \in C^{m}$. For operators of the form (3.10) we have the following form of the energy inequality: 
Lemma 2. Let $R(h)$ be of the form (3.10) and suppose $-\infty<a<b<+\infty$. Then there exists $C=C(\delta)>0$ such that

$$
\begin{aligned}
\frac{C_{2}}{2}\|u(b)\|_{0}^{2}-\frac{C_{1}}{2}\|u(a)\|_{0}^{2}+\frac{C_{2} \delta}{8} \int_{a}^{b} d t & \int_{R^{n}}|\xi|^{2 k}|\tilde{u}(\xi, t)|^{2} d \xi+C_{2}(\lambda-C) \int_{a}^{b}\|u(t)\|_{0}^{2} d t \\
& \leqq \operatorname{Re} \int_{a}^{b}\left(N(h)^{-1}(R(h)+\lambda I) u(t), N(h)^{-1} u(t)\right)_{0} d t
\end{aligned}
$$

for all $u \in\left\{C_{0}^{\infty}\left(R^{n+1}\right)\right\}^{m}$ and $\lambda>0$, with $C_{1}, C_{2}$ as in (3.11).

Proof. Let $N \equiv N(h)$ and $R \equiv R(h)$ and for $u \in\left\{C_{0}^{\infty}\left(R^{n+1}\right)\right\}^{m}$ let $v(t)=N^{-1} u(t)$. Since $N^{-1}(R+\lambda I) u(t)=\left(\partial / \partial t-\Lambda^{2 k} N^{-1} h N-N^{-1} J N+\lambda I\right) v(t)$, we obtain

$$
\begin{aligned}
\operatorname{Re} \int_{a}^{b}\left(N^{-1}(R+\lambda I) u(t),\right. & \left.N^{-1} u(t)\right)_{0} d t \\
= & \operatorname{Re} \int_{a}^{b}\left(\frac{\partial v}{\partial t}(t), v(t)\right)_{0} d t+\lambda \int_{a}^{b}\|v(t)\|_{0}^{2} d t \\
& -\operatorname{Re} \int_{a}^{b}\left(\Lambda^{2 k} N^{-1} h N v(t), v(t)\right)_{0} d t \\
& -\operatorname{Re} \int_{a}^{b}\left(N^{-1} J(t) N v(t), v(t)\right)_{0} d t .
\end{aligned}
$$

Let us estimate from below the last two terms in equation (3.12). Since $\operatorname{Re}\left(N^{-1} h N \zeta, \zeta\right) \leqq-(\delta / 4)|\zeta|^{2}$, we obtain, using Plancherel's Theorem,

$$
\begin{aligned}
-\operatorname{Re}\left(\Lambda^{2 k} N^{-1} h N v(t), v(t)\right)_{0} & =-\operatorname{Re}\left(N^{-1} h N \Lambda^{k} v(t), \Lambda^{k} v(t)\right)_{0} \\
& \geqq \frac{\delta}{4} \int_{a}^{b} d t \int_{R^{n}}|\xi|^{2 k}|\tilde{v}(\xi, t)|^{2} d \xi, \quad t \in R^{1} .
\end{aligned}
$$

Since $J(t)$ is uniformly of order $2 k-1$, we can apply (3.1), (3.11) and (3.2) to obtain, for arbitrary $\varepsilon>0$,

$$
\begin{aligned}
\left.\mid N^{-1} J(t) N v(t), v(t)\right)_{0} \mid & =\left|\left(J(t) N v(t),\left(N^{-1}\right)^{*} v(t)\right)_{0}\right| \\
& \leqq C\|J(t) N v(t)\|_{-k}\left\|\left(N^{-1}\right)^{*} v(t)\right\|_{k} \\
& \leqq C\|v(t)\|_{k-1}\|v(t)\|_{k} \leqq \varepsilon\|v(t)\|_{k}^{2}+C(\varepsilon)\|v(t)\|_{0}^{2}
\end{aligned}
$$

for all $t \in R^{1}$. Thus, for arbitrary $\varepsilon>0$, we obtain

$$
\left(N^{-1} J(t) N v(t), v(t)\right)_{0} \leqq\left(\varepsilon C_{k}\right) \int_{R^{n+1}}|\xi|^{2 k}|\tilde{v}(\xi, t)|^{2} d \xi+C(\varepsilon)\|v(t)\|_{0}^{2}
$$

for all $v \in\left\{C_{0}^{\infty}\left(R^{n+1}\right)\right\}^{m}$ and all $t \in R^{1}$. Combining (3.11), (3.13), (3.14) and letting $\varepsilon=\delta / 8 C_{k}$ our proof is complete.

In order to prove a similar energy inequality for operators $R$ of the form (3.9), we shall use a special partition of unity on $R^{n+1}$ and $\Sigma$ and certain estimates from Kohn and Nirenberg [6]. Since our symbols are not symbols in the sense of Kohn and Nirenberg, we shall have to modify their proof slightly. 
Proposition 2 (Compare With Theorem 5 of [6]). Let $k(x, t ; \xi)$ satisfy (3.4), let $K(t)=k(x, t ; D)$ and suppose $|k(x, t ; \xi)| \leqq \eta$ for all $\langle x, t\rangle \in R^{n}$ and $\xi \in \Sigma$. Then, for any $\varepsilon>0$ there exists $C(\varepsilon)>0$ such that

$$
\|K(t) \phi(t)\|_{0}^{2} \leqq(\eta+\varepsilon)^{2}\|\phi(t)\|_{0}^{2}+C(\varepsilon)\|\phi(t)\|_{-1}^{2}
$$

for all $t \in R^{1}$ and all $\phi \in C_{0}^{\infty}\left(R^{n+1}\right)$.

Proof. See $\S 8$ below.

Partitions of unity on $R^{n+1}$ and $\Sigma$. Let $h(x, t ; \xi)$ be given by (3.8). Since the coefficients of $P$ are uniformly continuous on $R^{n+1}$ and since $h(x, t ; \xi)$ is homogeneous in $\xi$ of degree zero we have that for any $\eta>0$ there exists $\gamma>0$ such that

$$
\|h(x, t ; \omega)-h(y, \tau ; \omega)\|<\eta / 2
$$

for $|x-y|^{2}+|t-\tau|^{2} \leqq \gamma^{2}$ and all $\omega \in \Sigma$. Let $d>0$ be such that $\sqrt{ }(n+1) d<\gamma / \sqrt{ } 2$. For each $(n+1)$-tuple of integers $(\alpha, \beta)=\left(\alpha_{1}, \alpha_{2}, \ldots, \alpha_{n}, \beta\right)$ let

$$
Q_{\alpha, \beta}=\left\{\langle x, t\rangle \in R^{n+1}:\left|x_{j}-d \alpha_{j}\right|<d,|t-d \beta|<d, j=1, \ldots, n\right\} .
$$

Choose $\zeta \in C_{0}^{\infty}\left(Q_{0,0}\right)$ such that $0 \leqq \zeta \leqq 1$ and $\sum_{(\alpha, \beta)}\{\zeta(x-d \alpha, t-d \beta)\}^{2} \equiv 1$ on $R^{n+1}$. Thus, if we define $\zeta_{\alpha, \beta}(x, t) \equiv \zeta(x-d \alpha, t-d \beta)$, then $\zeta_{\alpha, \beta} \in C_{0}^{\infty}\left(Q_{\alpha, \beta}\right)$ and $\sum_{(\alpha, \beta)} \zeta_{\alpha, \beta}^{2} \equiv 1$ on $R^{n+1}$. Enumerate the cubes $Q_{\alpha, \beta}$ and the corresponding functions $\zeta_{\alpha, \beta}$ in some order: $Q_{1}, Q_{2}, \ldots$ and $\zeta_{1}, \zeta_{2}, \ldots$ We remark that the cubes $Q_{i}$ overlap in such a fashion that any fixed point in $R^{n+1}$ is contained in exactly $2^{n+1}$ distinct cubes except for points on $\bigcup_{i} \partial Q_{i}$, a set of measure zero. Thus

$$
\sum_{i} \zeta_{i}(x, t)^{2} \equiv 1, \quad\langle x, t\rangle \in R^{n+1}
$$

and for any nonnegative integer $m$ there exists $C_{m}>0$ such that

$$
\sum_{|\alpha|+l<m} \sum_{i}\left|D^{\alpha}(\partial / \partial t)^{l} \zeta_{i}(x, t)\right|^{2} \leqq C_{m}
$$

for all $\langle x, t\rangle \in R^{n+1}$. Also, if we let $\left\langle x_{i}, t_{i}\right\rangle$ be the center of the cube $Q_{i}$, we see that

$$
\left\|h(x, t ; \xi)-h\left(x_{i}, t_{i} ; \xi\right)\right\|<\eta / 2
$$

for all $\langle x, t\rangle \in Q_{i}$ and all $\xi \in \Sigma$.

Since the coefficients of $P$ are bounded on $R^{n+1}$, we can find points $\xi_{1}, \xi_{2}, \ldots, \xi_{s}$ on $\Sigma$ and neighborhoods $\Omega_{1}, \Omega_{2}, \ldots, \Omega_{s}$ on $\Sigma$ of $\xi_{1}, \ldots, \xi_{s}$, respectively, such that

$$
\left\|h(x, t ; \xi)-h\left(x, t ; \xi_{j}\right)\right\|<\eta / 2
$$

for all $\langle x, t\rangle \in R^{n+1}$ and $\xi \in \Omega_{j}, j=1,2, \ldots, s$. Let $\phi_{1}, \phi_{2}, \ldots, \phi_{s}$ be functions defined on $\Sigma$ such that $0 \leqq \phi_{j} \leqq 1, \phi_{j} \in C_{0}^{\infty}\left(\Omega_{j}\right)$, and $\sum_{j=1}^{s} \phi_{j}(\omega)^{2} \equiv 1$ on $\Sigma$. Extend $\phi_{j}$ to all of $R^{n} \sim\{0\}$ so that $\phi_{j}$ is homogeneous of degree zero. On $R^{n}$ we define the operators $\Phi_{j} \equiv \phi_{j}(D), j=1, \ldots, s$. By Parseval's formula we have that

$$
\sum_{i, j}\left(\zeta_{i}(t) \Phi_{j} u, \zeta_{i}(t) \Phi_{j} v\right)_{0}=\sum_{j=1}^{s}\left(\Phi_{j} u, \Phi_{j} v\right)_{0}=(u, v)_{0}
$$

for all $t \in R^{1}$ and $u, v \in H^{0}$. 
RemarK. Since the choice of $\left\{Q_{i}\right\},\left\{\zeta_{i}\right\},\left\{\Omega_{j}\right\},\left\{\phi_{j}\right\}$ and $s$ depends on the number $\eta$ satisfying (3.15) we have that for each nonnegative integer $k$ there exists $\widetilde{C}=\tilde{C}(k, \eta)>0$ such that

$$
\sum_{|\alpha|+l \leqq k} \sum_{i}\left|D^{\alpha}(\partial / \partial t)^{l} \zeta_{i}(x, t)\right|^{2} \leqq \tilde{C}
$$

for all $\langle x, t\rangle \in R^{n+1}$; using Leibnitz's rule we obtain

$$
\sum_{i, j}\left\|\zeta_{i}(t) \Phi_{j} u\right\|_{k}^{2} \leqq \tilde{C}(k, \eta) \sum_{j=1}\left\|\Phi_{j} u\right\|_{k}^{2}=\tilde{C}(k, \eta)\|u\|_{k}^{2}
$$

for all $t \in R^{1}$ and $u \in H^{k}$. To estimate $\sum_{i}\left\|\zeta_{i}(t) u\right\|_{-k}^{2}$ in terms of $\|u\|_{-k}^{2}, k$ a positive integer, we use the following

Proposition 3. Let $k$ be a positive integer and suppose $\left\{\zeta_{i}\right\}_{i=1}^{\infty}$ is a set of functions satisfying:

(a) $\zeta_{i} \in C_{0}^{\infty}\left(R^{n}\right), i=1,2, \ldots$,

(b) for each positive integer $m$ there exists $C_{m}>0$ such that $\sum_{i}\left|D^{\alpha} \zeta_{i}(x)\right|^{2} \leqq C_{m}$ for all $|\alpha| \leqq m$ and $x \in R^{n}$.

Then there exists $C>0$ such that $\sum_{i}\left\|\zeta_{i} u\right\|_{-k}^{2} \leqq C\|u\|_{-k}^{2}$ for all $u \in H^{-k}$, in fact,

$$
C=C_{k} \sum_{|\alpha| \leqq k} \sup _{x \in R^{n}} \sum_{i}\left|D^{\alpha} \zeta_{i}(x)\right|^{2} .
$$

Proof. See $\S 8$ below.

Let $h_{i j}=h\left(x_{i}, t_{i} ; \xi_{j}\right), i=1,2, \ldots, j=1,2, \ldots, s$, where $h(x, t ; \xi)$ is given by (3.8). Define $R^{i j}$ by

$$
R^{i j}=\partial / \partial t-h_{i j} \Lambda^{2 k}-J(t)
$$

where $J(t)$ is given by (3.7). Define $N_{i j}=N\left(h_{i j}\right)$ (see Theorem 1). We now state and prove our first generalization of the classical energy inequality for operators of the form $\partial / \partial t-H(t) \Lambda^{2 k}-J(t)$ (see (3.9)).

TheOREM 2. Let $R$ be of the form (3.9) and let $-\infty<a<b<+\infty$. Then there exist constants $C^{\prime}(\delta)$ and $C^{\prime \prime}(\delta)>0$ such that

$$
\begin{aligned}
& \frac{C_{2}}{2}\|u(b)\|_{0}^{2}-\frac{C_{1}}{2}\|u(a)\|_{0}^{2} \\
& \quad+C^{\prime}(\delta) \int_{a}^{b} d t \int_{R^{n}}|\xi|^{2 k}|\tilde{u}(\xi, t)|^{2} d \xi+C_{2}\left(\lambda-C^{\prime \prime}(\delta)\right) \int_{a}^{b}\|u(t)\|_{0}^{2} d t \\
& \leqq \sum_{i, j} \operatorname{Re} \int_{a}^{b}\left(N_{i j}^{-1} \zeta_{i}(t) \Phi_{j}(R+\lambda I) u(t), N_{i j}^{-1} \zeta_{i}(t) \Phi_{j} u(t)\right)_{0} d t
\end{aligned}
$$

for all $u \in\left\{C_{0}^{\infty}\left(R^{n+1}\right)\right\}^{m}$ and all $\lambda>1 / C_{2}$.

Proof. We have $R=\partial / \partial t-H(t)-J(t)$ where $J(t)$ and $H(t)$ are given by (3.7) and (3.8), respectively. Consider the sesquilinear form $A$ defined by

$$
\begin{aligned}
A[u, v]= & \frac{C_{2}}{2}(u(b), v(b))_{0}-\frac{C_{1}}{2}(u(a), v(a))_{0} \\
& +\frac{C_{2} \delta}{8} \int_{a}^{b} d t \int_{R^{n}}|\xi|^{2 \kappa} \tilde{u}(\xi, t) \overline{\tilde{v}(\xi, t)} d \xi+C_{2}(\lambda-C) \int_{a}^{b}(u(t), v(t))_{0} d t
\end{aligned}
$$


for $u, v \in\left\{C_{0}^{\infty}\left(R^{n+1}\right)\right\}^{m}$ where $\lambda>0$ is fixed, and the constants $C_{1}, C_{2}$ and $C$ are those from Lemma 2. By Lemma 2 we know that for each $i$ and $j, i=1,2, \ldots$, $j=1, \ldots, s$,

$$
A[u, u] \leqq \operatorname{Re} \int_{a}^{b}\left(N_{i j}^{-1}\left(R^{i j}+\lambda I\right) u(t), N_{i j}^{-1} u(t)\right)_{0} d t
$$

for all $u \in\left\{C_{0}^{\infty}\left(R^{n+1}\right)\right\}^{m}$. Since $\Phi_{j} u(t) \in C^{\infty}$ for all $u \in\left\{C_{0}^{\infty}\left(R^{n+1}\right)\right\}^{m}$, we obtain

$$
A\left[\zeta_{i} \Phi_{j} u, \zeta_{i} \Phi_{j} u\right] \leqq \operatorname{Re} \int_{a}^{b}\left(N_{i j}^{-1}\left(R^{i j}+\lambda I\right) \zeta_{i} \Phi_{j} u(t), N_{i j}^{-1} \zeta_{i} \Phi_{j} u(t)\right)_{0} d t
$$

for all $u \in\left\{C_{0}^{\infty}\left(R^{n+1}\right)\right\}^{m}$ (here $\zeta_{i} \Phi_{j}$ denotes the operator $\zeta_{i} \cdot \Phi_{j}$ ).

We shall estimate $\sum_{i, j} A\left[\zeta_{i} \Phi_{j} u, \zeta_{i} \Phi_{j} u\right]$ from above by the right side of (3.22) and an "error" term. By subtracting this "error" term from a certain lower estimate on $\sum_{i, j} A\left[\zeta_{i} \Phi_{j} u, \zeta_{i} \Phi_{j} u\right]$ we shall arrive, with the appropriate choice of constants $C^{\prime}$ and $C^{\prime \prime}$, at inequality (3.22).

A trivial computation yields

$$
\begin{aligned}
R^{i j} \zeta_{i} \Phi_{j}= & \zeta_{i} \Phi_{j} R+\left[\frac{\partial}{\partial t}, \zeta_{i}\right] \Phi_{j}-h_{i j}\left[\Lambda^{2 k}, \zeta_{i}\right] \Phi_{j} \\
& +\zeta_{i}\left[\Phi_{j}, H(t)\right] \Lambda^{2 k}+\left[\zeta_{i} \Phi_{j}, J(t)\right]+\zeta_{i}\left(H(t)-h_{i j}\right) \Lambda^{2 k} \Phi_{j},
\end{aligned}
$$

where $[B, C]$ denotes the commutator of $B$ and $C$, i.e., $[B, C]=B C-C B$. We remark that, by (3.11), $\left\|N_{i j}^{-1}\right\| \leqq C_{1}$ for all $i$ and $j$.

Consider the following estimates corresponding to the last five terms on the right side of (3.23).

(1) Since $\left[\partial / \partial t, \zeta_{i}\right]=\partial \zeta_{i} / \partial t$ we have, by $(3.20)$,

$$
\begin{aligned}
\sum_{i, j} \int_{a}^{b} \mid\left(N_{i j}^{-1}\left[\partial / \partial t, \zeta_{i}\right] \Phi_{j} u,\right. & \left.N_{i j}^{-1} \zeta_{i} \Phi_{j} u\right)_{0} \mid d t \\
& \leqq C_{1} \sum_{i, j} \int_{a}^{b}\left\|\frac{\partial \zeta_{i}}{\partial t}(t) \Phi_{j} u(t)\right\|_{0}^{2} d t+C_{1} \sum_{i, j} \int_{a}^{b}\left\|\zeta_{i}(t) \Phi_{j} u(t)\right\|_{0}^{2} d t \\
& \leqq \widetilde{C}(\eta) \int_{a}^{b}\|u(t)\|_{0}^{2} d t, \quad u \in\left\{C_{0}^{\infty}\left(R^{n+1}\right)\right\}^{m} .
\end{aligned}
$$

(2) Corresponding to the third term on the right side of (3.23) we observe that

$$
\left[\Lambda^{2 k}, \zeta_{i}\right]=\sum_{|\alpha|=k} \sum_{0<\beta \leqq 2 \alpha} a_{\alpha}\left(\begin{array}{c}
2 \alpha \\
\beta
\end{array}\right) D^{\beta} \zeta_{i} \cdot D^{2 \alpha-\beta},
$$

where the $a_{\alpha}$ 's are positive integers. Applying Theorem 1, Proposition 2, (3.20) and (3.21) we obtain, for arbitrary $\varepsilon>0$,

$$
\begin{aligned}
\sum_{i, j} \int_{a}^{b}\left|\left(N_{i j}^{-1} h_{i j}\left(D^{\beta} \zeta_{i}\right) \Phi_{j} D^{2 \alpha-\beta} u, N_{i j}^{-1} \zeta_{i} \Phi_{j} u\right)_{0}\right| d t \\
\leqq C \sum_{i, j} \int_{a}^{b}\left\|\left(D^{\beta} \zeta_{i}\right) \Phi_{j} D^{2 \alpha-\beta} u\right\|_{-k}\left\|\zeta_{i} \Phi_{j} u\right\|_{k} d t \\
\leqq \varepsilon \int_{a}^{b}\|u(t)\|_{k}^{2} d t+\widetilde{C}(\eta) C(\varepsilon) \int_{a}^{b}\|u(t)\|_{k-1}^{2} d t,
\end{aligned}
$$


where $|\alpha|=k, 0<\beta \leqq 2 \alpha$. Thus, for arbitrary $\varepsilon>0$,

$\sum_{i, j} \int_{a}^{b}\left|\left(N_{i j}^{-1} h_{i j}\left[\Lambda^{2 k}, \zeta_{i}\right] \Phi_{j} u, N_{i j}^{-1} \zeta_{i} \Phi_{j} u\right)_{0}\right| d t \leqq \varepsilon \int_{a}^{b}\|u(t)\|_{k}^{2} d t+\widetilde{C}(\eta) C(\varepsilon) \int_{a}^{b}\|u(t)\|_{k-1}^{2} d t$ for all $u \in\left\{C_{0}^{\infty}\left(R^{n+1}\right)\right\}^{m}$.

(3) For the fourth term on the right side of (3.23) we apply Proposition 3, (3.1), (3.20) and (3.22) to obtain, for arbitrary $\varepsilon>0$,

$$
\begin{aligned}
\sum_{i, j}\left|\left(N_{i j}^{-1} \zeta_{i}(t)\left[\Phi_{j}, H(t)\right] \Lambda^{2 k} u(t), N_{i j}^{-1} \zeta_{i}(t) \Phi_{j} u(t)\right)_{0}\right| \\
\quad \leqq \varepsilon\|u(t)\|_{k}^{2}+C(\varepsilon) \widetilde{C}(\eta) \sum_{j=1}^{s}\left\|\left(\Phi_{j} H(t)-H(t) \Phi_{j}\right) \Lambda^{2 k} u(t)\right\|_{-k}^{2}
\end{aligned}
$$

for all $t \in R^{1}, u \in\left\{C_{0}^{\infty}\left(R^{n+1}\right)\right\}^{m}$. By Proposition 1(ii), the commutator $\Phi_{j} H(t)$ $-H(t) \Phi_{j}$ is uniformly of order -1 for $t \in R^{1}$ and we obtain, applying (3.5), that

$$
\sum_{j=1}^{s}\left\|\left(\Phi_{j} H(t)-H(t) \Phi_{j}\right) \Lambda^{2 k} u(t)\right\|_{-k}^{2} \leqq \widetilde{C}(\eta)\|u(t)\|_{k-1}^{2}
$$

for all $t \in R^{1}$. Thus, for arbitrary $\varepsilon>0$,

$\sum_{i, j} \int_{a}^{b}\left|\left(N_{i j}^{-1} \zeta_{i}\left[\Phi_{j}, H(t)\right] \Lambda^{2 k} u, N_{i j}^{-1} \zeta_{i} \Phi_{j} u\right)_{0}\right| d t$

$$
\leqq \varepsilon \int_{a}^{b}\|u(t)\|_{k}^{2} d t+C(\varepsilon) \widetilde{C}(\eta) \int_{a}^{b}\|u(t)\|_{k-1}^{2} d t
$$

for all $u \in\left\{C_{0}^{\infty}\left(R^{n+1}\right)\right\}^{m}$.

(4) For the fifth term on the right side of (3.23) we apply (3.1), (3.22), Proposition 3 and the fact $J(t)$ is uniformly of order $2 k-1$ to obtain, for arbitrary $\varepsilon>0$,

$\sum_{i, j} \int_{a}^{b}\left|\left(N_{i j}^{-1}\left[\zeta_{i} \Phi_{j}, J(t)\right] u, N_{i j}^{-1} \zeta_{i} \Phi_{j} u\right)_{0}\right| d t$

$$
\begin{aligned}
& \leqq \varepsilon \int_{a}^{b}\|u(t)\|_{k}^{2} d t+\widetilde{C}(\eta) C(\varepsilon) \sum_{i, j} \int_{a}^{b}\left\|\left[\zeta_{i} \Phi_{j}, J(t)\right] u(t)\right\|_{-k}^{2} d t \\
& \leqq \varepsilon \int_{a}^{b}\|u(t)\|_{k}^{2} d t+\widetilde{C}(\eta) C(\varepsilon) \int_{a}^{b}\|u(t)\|_{k-1}^{2} d t
\end{aligned}
$$

for all $u \in\left\{C_{0}^{\infty}\left(R^{n+1}\right)\right\}^{m}$.

(5) Corresponding to the sixth term on the right side of (3.23) we observe that $\Lambda^{2 k}=\sum_{|\alpha|=k} a_{\alpha} D^{2 \alpha}$, where the $a_{\alpha}$ 's are positive integers. Using integration by parts we obtain, for $|\alpha|=k$,

$$
\begin{aligned}
& \left|\left(N_{i j}^{-1} \zeta_{i}(t)\left(H(t)-h_{i j}\right) \Phi_{j} D^{2 \alpha} u(t), N_{i j}^{-1} \zeta_{i}(t) \Phi_{j} u(t)\right)_{0}\right| \\
& \leqq \\
& \left|\left(N_{i j}^{-1} \zeta_{i}(t)\left(H(t)-h_{i j}\right) \Phi_{j} D^{\alpha} u(t), N_{i j}^{-1} \zeta_{i}(t) \Phi_{j} D^{\alpha} u(t)\right)_{0}\right| \\
& + \\
& + \text { "a finite sum of remainder terms of the form } \\
& \left|\left(N_{i j}^{-1} D^{\delta} \zeta_{i}(t)\left(H(t)-h_{i j}\right) \Phi_{j} D^{\alpha} u(t), N_{i j}^{-1} D^{o} \zeta_{i}(t) \Phi_{j} D^{\gamma} u(t)\right)_{0}\right| \text { and } \\
& \left|\left(N_{i j}^{-1} D^{\delta} \zeta_{i}(t) \partial^{\tau} H(t) \Phi_{j} D^{\alpha} u(t), N_{i j}^{-1} D^{o} \zeta_{i}(t) \Phi_{j} D^{\gamma} u(t)\right)_{0}\right|, "
\end{aligned}
$$


where $|\delta|,|\tau|,|\rho| \leqq k-1,0<|\tau|,|\gamma| \leqq k-1$ and $\partial^{\tau} H(t)$ is the matrix of singular integral operators whose symbol is given by $\left(D_{x}^{\tau} h\right)(x, t ; \xi),\langle x, t ; \xi\rangle \in R^{n+1} \times \Sigma$. It is clear that the number of nonzero remainder terms depends only on $k$.

We now estimate the second type of remainder terms in (3.24). Since $|\gamma| \leqq k-1$, we can apply Proposition 1 and (3.5), (3.1) and (3.20) to obtain, for arbitrary $\varepsilon>0$, $\sum_{i, j}\left|\left(N_{i j}^{-1} D^{\delta} \zeta_{i}(t) \partial^{\tau} H(t) \Phi_{j} D^{\alpha} u(t), N_{i j}^{-1} D^{\rho} \zeta_{i}(t) \Phi_{j} D^{\gamma} u(t)\right)_{0}\right|$

$$
\leqq \varepsilon\|u(t)\|_{k}^{2}+C(\varepsilon) \widetilde{C}(\eta)\|u(t)\|_{k-1}^{2} \text { for all } t \in R^{1} .
$$

Now consider the second type of remainder term in (3.24). We observe that since the coefficients of $P$ are uniformly bounded, there is a constant $C=C(P)>0$ such that $\left\|h_{i j}\right\| \leqq C$ for all $i$ and $j$. Since $|\zeta-\eta|^{2} \leqq 2|\zeta|^{2}+2|\eta|^{2}$ for all $\zeta, \eta \in C^{m}$, we can use the fact that $H(t)$ is uniformly of order zero along with (3.5) to obtain for $|\alpha|$ $=k,|\gamma| \leqq k-1$ and arbitrary $\varepsilon>0$,

$$
\begin{aligned}
\sum_{i, j} \mid\left(N_{i j}^{-1} D^{\delta} \zeta_{i}(t)\left(H(t)-h_{i j}\right) \Phi_{j} D^{\alpha} u(t),\right. & \left.N_{i j}^{-1} D^{\rho} \zeta_{i}(t) \Phi_{j} D^{\gamma} u(t)\right)_{0} \mid \\
& \leqq \widetilde{C}(\eta)\|u(t)\|_{k}\left\{\sum_{i, j}\left\|D^{\rho} \zeta_{i}(t) \Phi_{j} D^{\gamma} u(t)\right\|_{0}^{2}\right\}^{1 / 2} \\
& \leqq \varepsilon\|u(t)\|_{k}^{2}+C(\varepsilon) \widetilde{C}(\eta)\|u(t)\|_{k-1}^{2}, \quad t \in R^{1}
\end{aligned}
$$

Thus

$$
\begin{aligned}
& \sum_{i, j} \int_{a}^{b}\left|\left(N_{i j}^{-1} \zeta_{i}\left(H(t)-h_{i j}\right) \Phi_{j} D^{2 \alpha} u, N_{i j}^{-1} \zeta_{i} \Phi_{j} u\right)_{0}\right| d t \\
& \leqq \varepsilon \int_{a}^{b}\|u(t)\|_{k}^{2} d t+C(\varepsilon) \tilde{C}(\eta) \int_{a}^{b}\|u(t)\|_{k-1}^{2} d t \\
& \quad+C_{k} \sum_{|\alpha|=k} \sum_{i, j} \int_{a}^{b}\left|\left(N_{i j}^{-1} \zeta_{i}\left(H(t)-h_{i j}\right) \Phi_{j} D^{\alpha} u, N_{i j}^{-1} \zeta_{i} \Phi_{j} D^{\alpha} u\right)_{0}\right| d t
\end{aligned}
$$

Let us estimate the last term in the right side of (3.25). We have that $H(t)-h_{i j}$ $=h(x, t ; D)-h_{i j}$. Let $h-h_{i j}=k_{j}^{\prime}+s_{i j}$ where

$$
k_{j}^{\prime}(x, t ; \xi)=h(x, t ; \xi)-h\left(x, t ; \xi_{j}\right), \quad s_{i j}(x, t)=h\left(x, t ; \xi_{j}\right)-h\left(x_{i}, t_{i} ; \xi_{j}\right) .
$$

Thus $H(t)-h_{i j}=K_{j}^{\prime}(t)+s_{i j}(t)$. where $K_{j}^{\prime}(t)=k_{j}^{\prime}(x, t ; D)$. For each $j, 1 \leqq j \leqq s$, let $\psi_{j} \in C_{0}^{\infty}\left(\Omega_{j}\right)$ with $\psi_{j} \equiv 1$ on the support of $\phi_{j}$ with $0 \leqq \psi_{j} \leqq 1$. Extend $\psi_{j}$ to all of $R^{n} \sim\{0\}$ so that $\psi_{j}$ is homogeneous of degree zero. Define $k_{j}(x, t ; \xi)$ $\equiv \psi_{j}(\xi) k_{j}^{\prime}(x, t ; \xi)$ for $\langle x, t\rangle \in R^{n+1}, \xi \in R^{n} \sim\{0\}, j=1, \ldots, s$. If we let $K_{j}(t)$ $=k_{j}(x, t ; D)$, then

$$
K_{j}^{\prime}(t) \Phi_{j}=K_{j}(t) \Phi_{j}, \quad j=1, \ldots, s .
$$

Now since, by (3.19), $\left|k_{j}(x, t ; \xi)\right| \leqq \eta / 2$ for all $\langle x, t ; \xi\rangle \in R^{n+1} \times \Sigma$, we can apply Proposition 2 to obtain, for arbitrary $\rho>0$,

$$
\left\|K_{j}(t) \Phi_{j} D^{\alpha} u(t)\right\|_{0}^{2} \leqq(\eta / 2+\rho)^{2}\left\|\Phi_{j} D^{\alpha} u(t)\right\|_{0}^{2}+C(\rho)\left\|\Phi_{j} D^{\alpha} u(t)\right\|_{-1}^{2}
$$


for $j=1, \ldots, s$ and $t \in R^{1}$. Letting $\rho=\eta / 2$ in (3.27) and using (3.26) we obtain, for arbitrary $\varepsilon>0$,

$$
\begin{aligned}
& \sum_{i, j}\left|\left(N_{i j}^{-1} \zeta_{i}(t) K_{j}^{\prime}(t) \Phi_{j} D^{\alpha} u(t), N_{i j}^{-1} \zeta_{i}(t) \Phi_{j} D^{\alpha} u(t)\right)_{0}\right| \\
&=\sum_{i, j}\left|\left(N_{i j}^{-1} \zeta_{i}(t) K_{j}(t) \Phi_{j} D^{\alpha} u(t), N_{i j}^{-1} \zeta_{i}(t) \Phi_{j} D^{\alpha} u(t)\right)_{0}\right| \\
& \quad \leqq \varepsilon\left\|D^{\alpha} u(t)\right\|_{0}^{2}+C(\varepsilon) \sum_{j=1}^{s}\left\|K_{j}(t) \Phi_{j} D^{\alpha} u(t)\right\|_{0}^{2} \\
& \leqq\left(\eta^{2}+\varepsilon\right)\|u(t)\|_{k}^{2}+C(\eta) C(\varepsilon)\|u(t)\|_{k-1}^{2}
\end{aligned}
$$

for each $t \in R^{1}$. We now consider the terms involving the multiplications $s_{i j}$.

By (3.18) we obtain

$$
\begin{aligned}
\sum_{i, j} \| \zeta_{i}(t) & s_{i j}(t) \Phi_{j} D^{\alpha} u(t) \|_{0}^{2} \\
& =\sum_{i, j} \int_{R^{n}} \zeta_{i}(x, t)^{2}\left\|h\left(x, t ; \xi_{j}\right)-h\left(x_{i}, t_{i} ; \xi_{j}\right)\right\|^{2}\left|\Phi_{j} D^{\alpha} u(x, t)\right|^{2} d x \\
& \leqq\left(\eta^{2} / 4\right)\left\|D^{\alpha} u(t)\right\|_{0}^{2}, \quad t \in R^{1} .
\end{aligned}
$$

Applying the Cauchy-Schwarz inequality and (3.28) we obtain, for $|\alpha|=k$,

$$
\sum_{i, j}\left|\left(N_{i j}^{-1} \zeta_{i}(t) s_{i j}(t) \Phi_{j} D^{\alpha} u(t), N_{i j}^{-1} \zeta_{i}(t) \Phi_{j} D^{\alpha} u(t)\right)_{0}\right| \leqq C \cdot \eta\|u(t)\|_{k}^{2},{ }_{t \in R^{1}}
$$

Combining the above estimate with (3.25) and (3.29) we obtain, for arbitrary $\varepsilon>0$,

$$
\begin{aligned}
\sum_{i, j} \int_{a}^{b} \mid\left(N_{i j}^{-1} \zeta_{i}\left(H(t)-h_{i j}\right) \Phi_{j} D^{2 \alpha} u,\right. & \left.N_{i j}^{-1} \zeta_{i} \Phi_{j} u\right)_{0} \mid d t \\
& \leqq C\{\varepsilon+\eta\} \int_{a}^{b}\|u(t)\|_{k}^{2} d t+C(\varepsilon) \tilde{C}(\eta) \int_{a}^{b}\|u(t)\|_{k-1}^{2} d t
\end{aligned}
$$

for all $u \in\left\{C_{0}^{\infty}\left(R^{n+1}\right)\right\}^{m}$, where $C$ depends on $k$ and $m$. Thus, for arbitrary $\varepsilon>0$, we obtain

$$
\begin{aligned}
\sum_{i, j} A\left[\zeta_{i} \Phi_{j} u, \zeta_{i} \Phi_{j} u\right] & \\
\leqq & \sum_{i, j} \operatorname{Re} \int_{a}^{b}\left(N_{i j}^{-1} \zeta_{i} \Phi_{j}(R+\lambda I) u, N_{i j}^{-1} \zeta_{i} \Phi_{j} u\right)_{0} d t+C\{\varepsilon+\eta\} \int_{a}^{b}\|u(t)\|_{k}^{2} d t \\
& +C(\varepsilon) \tilde{C}(\eta) \int_{a}^{b}\|u(t)\|_{k-1}^{2} d t+\tilde{C}(\eta) \int_{a}^{b}\|u(t)\|_{0}^{2} d t
\end{aligned}
$$

for all $u \in\left\{C_{0}^{\infty}\left(R^{n+1}\right)\right\}^{m}$.

We shall now obtain a lower estimate on $\sum_{i, j} A\left[\zeta_{i} \Phi_{j} u, \zeta_{i} \Phi_{j} u\right]$ by an expression similar in form to the left side of (3.22). We use the following

LEMmA 3. There exist constants $C_{k}>0$ and $\widetilde{C}(\eta)>0$ (see (3.20)) such that

$$
C_{k} \int_{R^{n}}|\xi|^{2 k}|\tilde{u}(\xi, t)|^{2} d \xi-\tilde{C}(\eta)\|u(t)\|_{0}^{2} \leqq \sum_{i, j} \int_{R^{n}}|\xi|^{2 k}\left|\mathscr{F}_{x}\left(\zeta_{i}(t) \Phi_{j} u(t)\right)(\xi)\right|^{2} d \xi
$$

for all $t \in R^{1}$ and $u \in C_{0}^{\infty}\left(R^{n+1}\right)$ (for convenience we denote $\phi$ by $\left.\mathscr{F}_{x} \phi\right)$. 
Assuming Lemma 3 we see that there exists $C_{0}=C_{0}(\delta, \Delta, k, m)>0$ and $C_{0}^{\prime}$ $=C_{0}^{\prime}(\delta, \Delta, k, m, \eta)>0$ such that

$$
\begin{aligned}
\left(C_{2} / 2\right)\|u(b)\|_{0}^{2}-\left(C_{1} / 2\right)\|u(a)\|_{0}^{2} & \\
& +C_{0} \int_{a}^{b} d t \int_{R^{n}}|\xi|^{2 k}|\tilde{u}(\xi, t)|^{2} d \xi+C_{2}\left(\lambda-C_{0}^{\prime}\right) \int_{a}^{b}\|u(t)\|_{0}^{2} d t \\
\leqq & \sum_{i, j} A\left[\zeta_{i} \Phi_{j} u, \zeta_{i} \Phi_{j} u\right]
\end{aligned}
$$

for all $u \in\left\{C_{0}^{\infty}\left(R^{n+1}\right)\right\}^{m}$. Since

$$
\|u(t)\|_{k}^{2} \leqq C_{k} \int_{R^{n}}|\xi|^{2 k}|\tilde{u}(\xi, t)|^{2} d \xi+C_{k}\|u(t)\|_{0}^{2}
$$

for all $t \in R^{1}$ and $u \in C_{0}^{\infty}\left(R^{n+1}\right)$, combining (3.30), (3.31) with (3.1) we obtain, for arbitrary $\varepsilon>0$,

$$
\begin{aligned}
\left(C_{2} / 2\right)\|u(b)\|_{0}^{2}-\left(C_{1} / 2\right)\|u(a)\|_{0}^{2} & \\
& +C_{0} \int_{a}^{b} d t \int_{R^{n}}|\xi|^{2 k}|\tilde{u}(\xi, t)|^{2} d \xi+C_{2}\left(\lambda-C_{0}^{\prime}\right) \int_{a}^{b}\|u(t)\|_{0}^{2} d t \\
\leqq & \sum_{i, j} \operatorname{Re} \int_{a}^{b}\left(N_{i j}^{-1} \zeta_{i} \Phi_{j}(R+\lambda I) u, N_{i j}^{-1} \zeta_{i} \Phi_{j} u\right)_{0} d t \\
& +C[\varepsilon+\eta] \int_{a}^{b} d t \int_{R^{n}}|\xi|^{2 k}|\tilde{u}(\xi, t)|^{2} d \xi+C(\varepsilon) \tilde{C}(\eta) \int_{a}^{b}\|u(t)\|_{0}^{2} d t
\end{aligned}
$$

for all $u \in\left\{C_{0}^{\infty}\left(R^{n+1}\right)\right\}^{m}$, where $C$ depends on $k$ and $m$. Letting $\varepsilon=\eta=C_{0} / 3 C$ completes the proof of Theorem 2.

Proof of Lemma 4. Since

$$
C_{1} \sum_{|\alpha|=k} \xi^{2 \alpha} \leqq|\xi|^{2 k} \leqq C_{2} \sum_{|\alpha|=k} \xi^{2 \alpha}
$$

for all $\xi \in R^{n}$, we have that

$$
\int_{R^{n}}|\xi|^{2 k}\left|\mathscr{F}_{x}\left(\zeta_{i}(t) \Phi_{j} u(t)\right)(\xi)\right|^{2} d \xi \leqq C_{1} \sum_{|\alpha|=k}\left\|D^{\alpha}\left(\zeta_{i}(t) \Phi_{j} u(t)\right)\right\|_{0}^{2}, \quad t \in R^{1} .
$$

Applying the inequality $|z+w|^{2} \geqq|z|^{2}-2|z||w|$ for all $z, w \in C$ we obtain, using Leibnitz's rule,

$$
\left|D^{\alpha}\left(\zeta_{i} \Phi_{j} u\right)\right|^{2} \geqq\left|\zeta_{i} \Phi_{j} D^{\alpha} u\right|^{2}-C_{k} \sum_{0<\beta \leqq \alpha}\left|\zeta_{i} \Phi_{j} D^{\alpha} u\right|\left|D^{\beta} \zeta_{i} \Phi_{j} D^{\alpha-\beta} u\right| .
$$

For $|\xi|=k$ and $0<\beta \leqq \alpha$ we use (3.20) to obtain, for arbitrary $\varepsilon>0$, $\sum_{i, j}\left\|\zeta_{i}(t) \Phi_{j} D^{\alpha} u(t)\right\|_{0}\left\|D^{\beta} \zeta_{i}(t) \Phi_{j} D^{\alpha-\beta} u(t)\right\|_{0}$

$$
\leqq \varepsilon \int_{R^{n}}|\xi|^{2 k}|\tilde{u}(\xi, t)|^{2} d \xi+C(\varepsilon) \tilde{C}(\eta)\|u(t)\|_{0}^{2}, \quad t \in R^{1} .
$$


Thus, for arbitrary $\varepsilon>0$,

$$
\begin{aligned}
& C_{1} \sum_{|\alpha|=k} \sum_{i, j}\left\|D^{\alpha}\left(\zeta_{i}(t) \Phi_{j} u(t)\right)\right\|_{0}^{2} \\
& \geqq C_{1} \sum_{|\alpha|=k}\left\|D^{\alpha} u(t)\right\|_{0}^{2}-\varepsilon \int_{R^{n}}|\xi|^{2 k}|\tilde{u}(\xi, t)|^{2} d \xi-C(\varepsilon) \tilde{C}(\eta)\|u(t)\|_{0}^{2}, \\
& \quad t \in R^{1}, u \in\left\{C_{0}^{\infty}\left(R^{n+1}\right)\right\}^{m} .
\end{aligned}
$$

Letting $\varepsilon=C_{1} / 2 C_{2}$ completes our proof.

\section{The energy inequality for distributors.}

Definition. Let $Q(\xi, \tau)=\left\{\tau^{2}+q^{4 k}(\xi)\right\}^{1 / 4 k}$ where $q(\xi)=\left\{1+|\xi|^{2}\right\}^{1 / 2}$, then for real $r$ and $s, k_{r, s}(\xi, \tau)=Q^{r}(\xi, \tau) q^{s}(\xi)$ is a temperate weight function.

$$
\begin{aligned}
\mathscr{H}^{r, s}=\left\{u \in \mathscr{S}^{\prime}\left(R^{n+1}\right):\right. & \hat{u} \in L_{\mathrm{loc}}^{\prime}\left(R^{n+1}\right) \text { and }\|u\|_{r, s}^{2} \\
& \left.=\int_{R^{n+1}} Q^{2 r}(\xi, \tau) q^{2 s}(\xi)|\hat{u}(\xi, \tau)|^{2} d \xi d \tau<\infty\right\}
\end{aligned}
$$

is a Hilbert space with the scalar product $(u, v)_{r, s}$ defined in the obvious way; here, we write

$$
\mathscr{F} u(\xi, \tau)=\hat{u}(\xi, \tau)=(2 \pi)^{-(n+1) / 2} \int_{R^{n+1}} e^{-i\langle\langle\xi, x\rangle+\tau t\}} u(x, t) d x d t
$$

when $u \in \mathscr{S}\left(R^{n+1}\right)$ and extend to $\mathscr{S}^{\prime}\left(R^{n+1}\right)$ in the usual way. We refer the reader to $\S 2$ of [5] for the various properties of $\mathscr{H}^{r, s}$ spaces. For $-\infty \leqq a<b \leqq+\infty$, we set $\Omega=\Omega_{a, b}=\left\{\langle x, t\rangle \in R^{n+1}: a<t<b\right\}$. Then $\mathscr{H}^{r, s}(\Omega) \equiv\left\{u \in \mathscr{S}^{\prime}(\Omega)\right.$ : there exists $v \in \mathscr{H}^{r, s}$ such that $\left.u=v \mid \Omega\right\}$ is a Banach space with the usual quotient norm: $\|u\|_{r, s, \Omega}=\inf \left\{\|v\|_{r, s}: v \in \mathscr{H}^{r, s}\right.$ and $\left.u=v \mid \Omega\right\}$. The spaces $\mathscr{H}^{r, s}(\Omega)$ are studied in $\S 4$ of [5].

Proposition 4. Let $p$ be a positive integer. $\mathscr{H}^{2 k p, 0}=\left\{u \in \mathscr{D}^{\prime}\left(R^{n+1}\right): u, D_{t}^{p} u\right.$, $D^{\alpha} u \in L^{2}\left(R^{n+1}\right)$ for all $\left.\alpha,|\alpha|=2 k p\right\}$ and $\|u\|_{2 k p, 0}$ is equivalent to the norm

$$
\left\{\sum_{|\alpha|=2 k p}\left\|D^{\alpha} u\right\|_{L^{2}\left(R^{n+1}\right)}^{2}+\left\|D_{t}^{p} u\right\|_{L^{2}\left(R^{n+1}\right)}^{2}+\|u\|_{L^{2}\left(R^{n+1}\right)}^{2}\right\}^{1 / 2} .
$$

Proof. The proof is a trivial consequence of the inequality

$$
\left\{\left(1+|\xi|^{2}\right)^{2 k}+\tau^{2}\right\}^{p} \leqq C_{k, p}\left\{1+|\xi|^{4 k p}+\tau^{2 p}\right\}
$$

for all $\langle\xi, \tau\rangle \in R^{n+1}$.

REMARK. If $|\alpha|+2 k j \leqq 2 k p$, then $D^{\alpha} D_{t}^{j}$ is bounded from $\mathscr{H}^{r, s}$ into $\mathscr{H}^{r-2 k p, s}$ for all real $r$ and $s$.

Proposition 5. Let $j$ and $p$ be integers with $j$ positive. Then $u \in \mathscr{H}^{-2 k j p, 0}$ if and only if $u$ has a representation

$$
u=u_{0}+\sum_{|\alpha|=2 k j} D^{\alpha} u_{\alpha}+D_{t}^{j} u_{j}
$$


where $u_{0} \in \mathscr{H}^{-2 k j(p-2), 0}, u_{\alpha} \in \mathscr{H}^{-2 k j(p-1), 0}$ for $|\alpha|=2 k j$, and $u_{j} \in \mathscr{H}^{-2 k j(p-1), 0}$, in such a way that $\|u\|_{-2 k j p, 0}$ is equivalent to

$$
\left\{\left\|u_{0}\right\|_{-2 k j(p-2), 0}^{2}+\sum_{|\alpha|=2 k j}\left\|u_{\alpha}\right\|_{-2 k j(p-1), 0}^{2}+\left\|u_{j}\right\|_{-2 k j(p-1), 0}^{2}\right\}^{1 / 2} .
$$

Proof. Define

$$
\sigma(\xi, \tau)=\left\{1+\sum_{|\alpha|=2 k j} \xi^{2 \alpha}+\tau^{2 j}\right\}^{-1}, \quad\langle\xi, \tau\rangle \in R^{n+1} .
$$

For $u \in \mathscr{H}^{-2 k j p, 0}$ we let $u_{0}=\sigma\left(D, D_{t}\right) u, u_{\alpha}=\left[D^{\alpha} \sigma\left(D, D_{t}\right)\right] u$ for each $\alpha,|\alpha|=2 k j$, and $u_{j}=\left[D_{t}^{j} \sigma\left(D, D_{t}\right)\right] u$. Our results follow by a simple calculation.

Notation. As in [5] we write

$$
[\phi, \psi]=\int_{R^{n+1}} \phi(x, t) \bar{\psi}(x, t) d x d t
$$

where $\phi, \psi \in C_{0}^{\infty}\left(R^{n+1}\right)$. By Proposition 3 of [5], $\mathscr{H}^{r, s}$ and $\mathscr{H}^{-r,-s}$ are dual Hilbert spaces, the duality being given by the sesquilinear form (which we again denote by $[]$,$) obtained by extension.$

Let $\left\{\psi_{i}\right\}_{i=1}^{\infty}$ be elements of $C_{0}^{\infty}\left(R^{n+1}\right)$ satisfying the following condition: for each nonnegative integer $m$ there exists $C_{m}>0$ such that

$$
\sum_{|\alpha|+j \leqq m} \sum_{i}\left|D^{\alpha}(\partial / \partial t)^{j} \psi_{i}(x, t)\right|^{2} \leqq C_{m}
$$

for all $\langle x, t\rangle \in R^{n+1}$. We recall from $\S 3$ that if $\left\{\zeta_{i}\right\}_{1}^{\infty}$, and $\left\{\rho_{i}\right\}_{1}^{\infty}$ satisfy (4.2) and if $l$ is a nonnegative integer, then

$$
\sum_{i}\left|\left(\zeta_{i}(t) \phi(t), \rho_{i}(t) \psi(t)\right)_{0}\right| \leqq C\|\phi(t)\|_{l}\|\psi(t)\|_{-l}
$$

for all $t \in R^{1}, \phi, \psi \in C_{0}^{\infty}\left(R^{n+1}\right)$ where $C>0$ depends upon $\left\{\zeta_{i}\right\}_{1}^{\infty},\left\{\rho_{i}\right\}_{1}^{\infty}$ and $l$. Integrating with respect to $t$, it is easily seen that

$$
\left|\sum_{i}\left[\zeta_{i} \phi, \rho_{i} \psi\right]\right| \leqq C\|\phi\|_{0, l}\|\psi\|_{0,-l}
$$

for all $\phi, \psi \in C_{0}\left(R^{n+1}\right)$. Thus $\sum_{i}\left[\zeta_{i} \cdot, \rho_{i} \cdot\right]$ extends uniquely to a continuous sesquilinear form on $\mathscr{H}^{0, l} \times \mathscr{H}^{0,-l}$ satisfying (4.3) with $\phi \in \mathscr{H}^{0, l}$ and $\psi \in \mathscr{H}^{0,-l}$. By Proposition 1 of [5], $\sum_{i}\left[\zeta_{i} u, \rho_{i} v\right]$ does not depend on $l$. We wish, however, to show that $\sum_{i}\left[\zeta_{i} \cdot, \rho_{i} \cdot\right]$ can be defined in a continuous way on $\mathscr{H}^{r, s} \times \mathscr{H}^{-r,-s}$ for general $r$ and $s$.

Proposition 6. Let $\left\{\zeta_{i}\right\}_{1}^{\infty}$ and $\left\{\rho_{i}\right\}_{1}^{\infty}$ satisfy condition (4.2) and suppose $r$ and $s$ are real numbers. Then the form $\sum_{i}\left[\zeta_{i} \phi, \rho_{i} \psi\right], \phi, \psi \in C_{0}^{\infty}\left(R^{n+1}\right)$, extends in a unique way to a continuous sesquilinear form on $\mathscr{H}^{r, s} \times \mathscr{H}^{-r,-s}$ (which we denote by $\left.\sum_{i}\left[\zeta_{i} u, \rho_{i} v\right]\right)$.

Proof. See $\S 8$ below. 
Using the notation of $[5, \S 4]$, we have that the mapping which assigns to $\phi \in C_{(0)}^{\infty}(\Omega)$ the function $t \rightarrow \phi(\cdot, t)$ taking $[a, b]$ into $C_{0}^{\infty}\left(R^{n}\right)$, extends, in a unique way, to a continuous embedding of $\mathscr{H}^{r, s}(\Omega)$ into $C\left([a, b]: H^{r-k+s}\right)$, provided $r>k$. For $u \in \mathscr{H}^{r, s}(\Omega), r>k$, and $t \in[a, b]$, we write $u(t)$ for $u(\cdot, t) \in H^{r-k+s}$. If $\phi \in C_{0}^{\infty}\left(R^{n+1}\right)$ define

$$
\begin{aligned}
H_{a} \phi(x, t) & =\phi(x, t) & & \text { if } t \geqq a, \\
& =0 & & \text { if } t<a .
\end{aligned}
$$

By Proposition 11 of [5], if $|r|<k$ then $H_{a} \phi \in \mathscr{H}^{r, s}$; moreover there exists $C=C_{r, s}$ $>0$ such that $\left\|H_{a} \phi\right\|_{r, s} \leqq C\|\phi\|_{r, s}$. Thus, $H_{a}$ defines a continuous projection of $\mathscr{H}^{r, s}$ onto $\mathscr{H}_{0}^{r, s}\left(\bar{\Omega}_{a,+\infty}\right)$ (see [5] for definition of $\mathscr{H}^{r, s}(K)$ where $K$ is closed in $R^{n+1}$ ).

Proposition 7 (Compare with Proposition 12 of [5]). Let $\left\{\zeta_{i}\right\}_{1}^{\infty}$ and $\left\{\rho_{i}\right\}_{1}^{\infty}$ satisfy (4.2). Then given real $r_{1}$ and $r_{2}$, satisfying $r_{1}+r_{2} \geqq 0$ and $\min \left(r_{1}, r_{2}\right)>-k$, any real $s_{1}$ and $s_{2}$, and $-\infty \leqq a<b \leqq+\infty$, the form

$$
\sum_{i} \int_{a}^{b}\left(\zeta_{i}(t) \phi(t), \rho_{i}(t) \psi(t)\right)_{0} d t, \quad \phi, \psi \in C_{(0)}^{\infty}(\bar{\Omega})
$$

extends in a unique way to a continuous sesquilinear form on $\mathscr{H}^{r_{1}, s_{1}}(\Omega) \times \mathscr{H}^{r_{2}, s_{2}}(\Omega)$ (which we denote by $\sum_{i} \int_{a}^{b}\left(\zeta_{i} u, \rho_{i} v\right)_{0} d t$ ), provided $r_{1}+s_{1}+r_{2}+s_{2} \geqq 0$.

Remark. Proposition 12 of [5] shows that the form $\int_{a}^{b}(\phi(t), \psi(t))_{\theta} d t, \phi, \psi \in C_{0}^{\infty}(\bar{\Omega})$, extends in a unique way to a continuous sesquilinear form on $\mathscr{H}^{r_{1}, s_{1}}(\Omega)$ $\times \mathscr{H}^{r_{2}, s_{2}}(\Omega)$ (which is denoted by $\int_{a}^{b}(u, v)_{\theta} d t$ ), provided that $\theta \leqq \frac{1}{2}\left(r_{1}+s_{1}+r_{2}+s_{2}\right)$.

Proof. Suppose first that $\phi, \psi \in C_{0}^{\infty}\left(R^{n+1}\right)$. Choose $r$ real such that $|r|<k$ and $-r_{2} \leqq r \leqq r_{1}$; then let $s=\frac{1}{2}\left(s_{1}+r_{1}-s_{2}-r_{2}\right)-r$ which implies that $-\left(s_{2}+r_{2}\right) \leqq r+s$ $\leqq s_{1}+r_{1}$. Thus, by Proposition 6,

$$
\begin{aligned}
\left|\sum_{i} \int_{a}^{b}\left(\zeta_{i}(t) \phi(t), \rho_{i}(t) \psi(t)\right)_{0} d t\right| & =\left|\sum_{i}\left[\left(H_{a}-H_{b}\right) \zeta_{i} \phi, \rho_{i} \psi\right]\right|=\left|\sum_{i}\left[\zeta_{i}\left(H_{a}-H_{b}\right) \phi, \rho_{i} \psi\right]\right| \\
& \leqq C\|\phi\|_{r, s}\|\psi\|_{-r,-s} \leqq C\|\phi\|_{r_{1}, s_{1}}\|\psi\|_{r_{2}, s_{2}} .
\end{aligned}
$$

Thus $\sum_{i} \int_{a}^{b}\left(\zeta_{i} u, \rho_{i} v\right)_{0} d t$ extends to a continuous sesquilinear form on $\mathscr{H}^{r_{1}, s_{1}}$

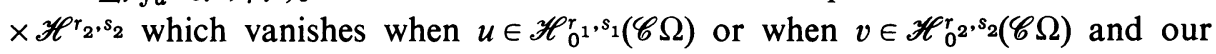
proof is complete.

Note. For $\rho_{i}=\zeta_{i}$ we write $\sum_{i} \int_{a}^{b}\left\|\zeta_{i} u\right\|_{0}^{2} d t$ for $\sum_{i} \int_{a}^{b}\left(\zeta_{i} u, \zeta_{i} u\right)_{0} d t$; if $\sum_{i} \zeta_{i}^{2} \equiv 1$ it is obvious that $\sum_{i} \int_{a}^{b}\left\|\zeta_{i} u\right\|_{0}^{2} d t=\|u\|_{0,0, \Omega}^{2}$. Also, for $\theta \leqq \frac{1}{2}\left(r_{1}+s_{1}+r_{2}+s_{2}\right)$ we write $\int_{a}^{b}\|u\|_{\theta}^{2} d t$ for $\int_{a}^{b}(u, u)_{\theta} d t$ (see Proposition 12 of [5]); it is easily seen that $\int_{a}^{b}\|u\|_{\theta}^{2} d t$ $=\|u\|_{0, \theta, \Omega}^{2}$.

Definition. Let $r$ and $s$ be real numbers. As in [5] $\mathscr{M}_{r, s}$ is the unitary isomorphism of $\mathscr{H}^{\rho, \sigma}$ onto $\mathscr{H}^{\rho-r, \sigma-s}$ (for each real $\rho$ and $\sigma$ ) given by $\mathscr{F}\left(\mathscr{M}_{r, s} \phi\right)(\xi, \tau)$ $=Q^{r}(\xi, \tau) q^{s}(\xi) \hat{\phi}(\xi, \tau)$, when $\phi \in C_{0}^{\infty}\left(R^{n+1}\right)$, and extended.

$\mathscr{M}_{0, \sigma}$ maps $\mathscr{H}^{r, s}(\Omega)$ continuously into $\mathscr{H}^{r, s-\sigma}(\Omega)$ for all real $r, s$, and $\sigma$ (see $\S 4$ of [5]). 
In the following theorem we take $\left\{\zeta_{i}\right\}_{1}^{\infty}$ to be the square partition of unity used in Theorem 2.

TheOrem 3 (COMPARE With TheOrem 3 of [5]). Let $R$ be given by (3.9). Given real $r$ and $s, r>k$, and $-\infty \leqq a<b \leqq+\infty$, there exists $C_{3}, C_{4}>0$ (depending on $\sigma, r$ and $s$ ) such that

$$
\begin{aligned}
\left(C_{2} / 2\right)\|u(b)\|_{r+s-k}^{2} & -\left(C_{1} / 2\right)\|u(a)\|_{r+s-k}^{2} \\
& \quad+C_{3} \int_{a}^{b}\|u\|_{r+s}^{2} d t+C_{2}\left(\lambda-C_{4}\right) \int_{a}^{b}\|u\|_{r+s-k}^{2} d t \\
\leqq & \operatorname{Re} \int_{a}^{b}\left(N_{i j}^{-1} \zeta_{i} \Phi_{j} \mathscr{M}_{0, \rho}(R+\lambda I) u, N_{i j}^{-1} \zeta_{i} \Phi_{j} \mathscr{M}_{0, \rho} u\right)_{0} d t
\end{aligned}
$$

for all $u \in\left\{\mathscr{H}^{r, s}(\Omega)\right\}^{m}$ and $\lambda>1 / C_{2}$, where $\rho=r+s-k$.

Proof. First suppose $r+s=k$. By Theorem 2 and (3.31), (4.4) holds for $u \in\left\{C_{0}^{\infty}\left(R^{n+1}\right)\right\}^{m}$ with $C_{3}=C^{\prime}(\delta) / C_{k}$ and $C_{4}=C^{\prime \prime}(\delta)+C^{\prime}(\delta) / C_{2}$. (4.4) extends to the case where $u \in\left\{\mathscr{H}^{r, s}(\Omega)\right\}^{m}$ by application of Proposition 9 of [5] and our Proposition 7 as follows:

$$
\sum_{i, j} \int_{a}^{b}\left(N_{i j}^{-1} \zeta_{i} u, N_{i j}^{-1} \zeta_{i} v\right)_{0} d t
$$

is continuous on $\left\{\mathscr{H}^{r-2 k, s}(\Omega)\right\}^{m} \times\left\{\mathscr{H}^{r, s}(\Omega)\right\}^{m}$ by Proposition 7 ; since $R:\left\{\mathscr{H}^{r, s}(\Omega)\right\}^{m}$ $\rightarrow\left\{\mathscr{H}^{r-2 k, s}(\Omega)\right\}^{m}$ is continuous it follows that

$$
\sum_{i, j} \int_{a}^{b}\left(N_{i j}^{-1} \zeta_{i} \Phi_{j}(R+\lambda I) u, N_{i j}^{-1} \zeta_{i} \Phi_{j} v\right)_{0} d t
$$

is continuous on $\left\{\mathscr{H}^{r, s}(\Omega)\right\}^{m} \times\left\{\mathscr{H}^{r, s}(\Omega)\right\}^{m}$.

Now let $r+s-k=\rho$. We have that $R \mathscr{M}_{0, \rho}=\mathscr{M}_{0, \rho} R+\left(\mathscr{M}_{0, \rho} H-H \mathscr{M}_{0, \rho}\right) \Lambda^{2 k}$ $+\left(\mathscr{M}_{0, \rho} J-J \mathscr{M}_{0, \rho}\right)$. Choosing $\theta$ between 0 and 1 , and applying our Proposition 7 and Proposition 5(ii) and Proposition 2(i) of [5], we obtain, for arbitrary $\varepsilon>0$,

$$
\begin{aligned}
\mid \sum_{i, j} \operatorname{Re} & \int_{a}^{b}\left(N_{i j}^{-1} \zeta_{i} \Phi_{j}\left[\mathscr{M}_{0, \rho} H-H \mathscr{M}_{0, \rho}\right] \Lambda^{2 k} u, N_{i j}^{-1} \zeta_{i} \Phi_{j} \mathscr{M}_{0, \rho} u\right)_{0} d t \mid \\
& \leqq C_{k}\left\|\left[\mathscr{M}_{0, \rho} H-H \mathscr{M}_{0, \rho}\right] \Lambda^{2 k} u\right\|_{0,-k, \Omega}\left\|\mathscr{M}_{0, \rho} u\right\|_{0, k, \Omega} \leqq C\|u\|_{0, \rho+k-\theta, \Omega}\|u\|_{0, k+\rho, \Omega} \\
& \leqq(\varepsilon / 2)\|u\|_{0, \rho+k, \Omega}^{2}+C(\varepsilon)\|u\|_{0, \rho, \Omega}^{2}=\frac{\varepsilon}{2} \int_{a}^{b}\|u\|_{\rho+k}^{2} d t+C(\varepsilon) \int_{a}^{b}\|u\|_{\rho}^{2} d t .
\end{aligned}
$$

A similar calculation yields, for arbitrary $\varepsilon>0$,

$$
\begin{aligned}
&\left|\sum_{i, j} \operatorname{Re} \int_{a}^{b}\left(N_{i j}^{-1} \zeta_{i} \Phi_{j}\left[\mathscr{M}_{0, \rho} J-J \mathscr{M}_{0, \rho}\right] u, N_{i j}^{-1} \zeta_{i} \Phi_{j} \mathscr{M}_{0, \rho} u\right)_{0} d t\right| \\
& \leqq \frac{\varepsilon}{2} \int_{a}^{b}\|u\|_{\rho+k}^{2} d t+C(\varepsilon) \int_{a}^{b}\|u\|_{\rho}^{2} d t .
\end{aligned}
$$

Let $\varepsilon=C_{3} / 2$ and our proof is complete. 


\section{Uniqueness of the Cauchy problem for $P$.}

THEOREM 4. Given real $r$ and $s, r>(2 m-1) k$, and $-\infty<a<b<+\infty$, the mapping $\phi \leadsto\left\langle P \phi, \phi(a),(\partial / \partial t) \phi(a), \ldots,(\partial / \partial t)^{m-1} \phi(a)\right\rangle$ is one-to-one from $\mathscr{H}^{r, s}(\Omega)$ into $\mathscr{H}^{r-2 k m}(\Omega) \oplus H^{r+s-k} \oplus H^{r+s-3 k} \oplus \cdots \oplus H^{r+s-(2 m-1) k}$, where $\Omega=\Omega_{a, b}$.

Proof. We write $\mathscr{P} \phi=\left\langle P \phi, \phi(a),(\partial \phi / \partial t)(a), \ldots,(\partial / \partial t)^{m-1} \phi(a)\right\rangle$ for $\phi \in \mathscr{H}^{r, s}(\Omega)$. For each $\phi \in \mathscr{H}^{r, s}(\Omega)$ let $u_{\phi}=\left\langle u_{1}, u_{2}, \ldots, u_{m}\right\rangle$ be defined by $u_{j}=\Lambda^{2 k(m-j)} D_{t}^{j-1} \phi$, $j=1,2, \ldots, m$; clearly $u_{\phi} \in\left\{\mathscr{H}^{r-2 k(m-1), s}(\Omega)\right\}^{m}$. By definition of $R$ (see $\S 3$ ) if $\phi \in \mathscr{H}^{r, s}(\Omega)$, then $f=P \phi \in \mathscr{H}^{r-2 k m, s}(\Omega)$ and $R\left(u_{\phi}\right)=i(f-Q \phi) \varepsilon_{m}$, where $\varepsilon_{m} \in R^{m}$ has 1 as its $m$ th component and zeros elsewhere. Throughout the remainder of this proof $C, C_{0}, C_{1}, C_{2}, C_{3}, C_{4}, C_{5}$ will denote positive constants depending only on $\Delta, \delta, k$ and $m$, not necessarily the same at each occurence.

If, in the energy inequality (4.4), we let $\lambda=\max \left\{2 C_{4}, 1 / C_{2}\right\}$ and replace $u$ by $e^{-\lambda t} u$, we obtain, for arbitrary $\varepsilon>0$,

$C_{2} \exp \left(-C_{0} b\right)\|u(b)\|_{\rho}^{2}-C_{1} \exp \left(-C_{0} a\right)\|u(a)\|_{\rho}^{2}$

$$
\begin{aligned}
& +C_{3} \exp \left(-C_{0} b\right) \int_{a}^{b}\|u\|_{\rho+k}^{2} d t+C_{5} \exp \left(-C_{0} b\right) \int_{a}^{b}\|u\|_{\rho}^{2} d t \\
\leqq & \varepsilon\left\|\mathscr{M}_{0, \rho} u\right\|_{0,0, \Omega}^{2}+C(\varepsilon) \exp \left(-C_{0} a\right)\left\|\mathscr{M}_{0, \rho}(f-Q \phi)\right\|_{0,0, \Omega}^{2}
\end{aligned}
$$

for $\phi \in \mathscr{H}^{r, s}(\Omega)$ with $u=u_{\phi}, f=P \phi$ and $\rho=r+s-(2 m-1) k$. If we choose $\varepsilon=C_{5} \exp \left(-C_{0} b\right) / 2$ in (5.1) we obtain

$$
\begin{array}{r}
C_{2}\|u(b)\|_{\rho}^{2}-C_{1} \exp \left(C_{0}(b-a)\right)\|u(a)\|_{\rho}^{2}+C_{3} \int_{a}^{b}\|u\|_{\rho+k}^{2} d t+\left(C_{5} / 2\right) \int_{a}^{b}\|u\|_{\rho}^{2} d t \\
\leqq C_{0} \exp \left(C_{0}(b-a)\right)\left\{\left\|\mathscr{M}_{0, \rho} Q \phi\right\|_{0,0, \Omega}^{2}+\|f\|_{0, \rho, \Omega}^{2}\right\} .
\end{array}
$$

Since

$$
Q=\sum_{j=1}^{m-1} \sum_{|\alpha| \leqq 2 k(m-j)-1} a_{\alpha, j-1}(x, t) D_{x}^{\alpha} D_{t}^{j-1}
$$

has its coefficients in $C_{B}^{\infty}\left(R^{n+1}\right)$, we have, for arbitrary small $\varepsilon>0$,

$$
\begin{aligned}
\left\|\mathscr{M}_{0, \rho} Q \phi\right\|_{0,0, \Omega}^{2} \leqq & \varepsilon \sum_{j=1}^{m-1} \sum_{|\alpha|=2 k(m-j)}\left\|D^{\alpha} D_{t}^{j-1}\left(\mathscr{M}_{0, \rho} \phi\right)\right\|_{0,0, \Omega}^{2} \\
& +C(\varepsilon) \sum_{j=1}^{m-1}\left\|D_{t}^{j-1}\left(\mathscr{M}_{0, \rho} \phi\right)\right\|_{0,0, \Omega}^{2}
\end{aligned}
$$

where $C(\varepsilon)$ is independent of $a$ and $b$, and $0<C\left(\varepsilon_{1}\right)<C\left(\varepsilon_{2}\right)$ if $\varepsilon_{1}>\varepsilon_{2}$. Using Poincaré's inequality:

$$
\int_{a}^{b}\|u(t)\|_{0}^{2} d t \leqq 2(b-a) \int_{a}^{b}\left\|D_{t} u(t)\right\|_{0}^{2} d t+2\|u(a)\|_{0}^{2}
$$

for all $u \in C_{(0)}^{\infty}(\bar{\Omega})$, and extended to $\mathscr{H}^{r, s}(\Omega)$, we obtain

$$
\begin{aligned}
& \sum_{j=1}^{m-1}\left\|D_{t}^{j-1}\left(\mathscr{M}_{0, \rho} \phi\right)\right\|_{0,0, \Omega}^{2} \\
& \quad \leqq \chi_{1}(b-a)\left\|D_{t}^{m-1}\left(\mathscr{M}_{0, \rho} \phi\right)\right\|_{0,0, \Omega}^{2}+\chi_{2}(b-a) \sum_{j=1}^{m-1}\left\|D_{t}^{j-1}\left(\mathscr{M}_{0, \rho} \phi\right)(a)\right\|_{0}^{2}
\end{aligned}
$$


where $\chi_{1}(b-a) \downarrow 0$ as $b \rightarrow a$. We observe that there exists $A_{i}=A_{i}(k, m)>0$, $i=1,2$, such that

$$
A_{1}\left\|u_{\phi}\right\|_{0,0}^{2} \leqq \sum_{j=1}^{m} \sum_{|\alpha|=2 k(m-j)}\left\|D^{\alpha} D_{t}^{j-1} \phi\right\|_{0,0}^{2} \leqq A_{2}\left\|u_{\phi}\right\|_{0,0}^{2}
$$

for all $\phi \in \mathscr{H}^{r, s}$ where $r+s=(2 m-1) k$. Applying (5.3), (5.4) and (5.5), we obtain, for arbitrary $\varepsilon>0$,

$$
\begin{aligned}
& \left\|\mathscr{M}_{0, \rho} Q \phi\right\|_{0,0, \Omega}^{2} \\
& \quad \leqq \varepsilon\left\|u_{\phi}\right\|_{0, \rho, \Omega}^{2}+C(\varepsilon)\left\{\chi_{1}(b-a)\left\|D_{t}^{m-1} \phi\right\|_{0, \rho, \Omega}^{2}+\chi_{2}(b-a) \sum_{j=1}^{m-1}\left\|D_{t}^{j-1} \phi(a)\right\|_{\rho}^{2}\right\} .
\end{aligned}
$$

Letting $\varepsilon=C_{5} \exp \left(-C_{0}(b-a)\right) / 4 C_{0}$ in (5.6), it follows from (5.3) that

$$
\begin{aligned}
& C_{2}\|u(b)\|_{\rho}^{2}-C_{1} \exp \left(C_{0}(b-a)\right)\|u(a)\|_{\rho}^{2}+C_{3} \int_{a}^{b}\|u\|_{\rho+k}^{2} d t+\left(C_{5} / 4\right) \int_{a}^{b}\|u\|_{\rho}^{2} d t \\
& \leqq \\
& \quad C_{a, b} C_{0} \exp \left(C_{0}(b-a)\right)\left\{\chi_{1}(b-a)\left\|D_{t}^{m-1} \phi\right\|_{0, \rho, \Omega}^{2}+\chi_{2}(b-a) \sum_{j=1}^{m-1}\left\|D_{t}^{j-1} \phi(a)\right\|_{\rho}^{2}\right\} \\
& \quad+C_{0} \exp \left(C_{0}(b-a)\right)\|f\|_{0, \rho, \Omega}^{2}
\end{aligned}
$$

for $\phi \in \mathscr{H}^{r, s}(\Omega)$ where $u=u_{\phi}, f=P \phi$ and $C_{a, b}=C(\varepsilon(b-a))$. We observe that if $0<\theta<b-a$ then $C(\varepsilon(\theta))<C_{a, b}$. Also, by (5.5) we have that $\left\|D_{t}^{m-1} \phi\right\|_{0, \rho, \Omega}^{2}$ $\leqq A_{2}\left\|u_{\phi}\right\|_{0, \rho, \Omega}^{2}, A_{2}$ independent of $a$ and $b$. Thus, by choosing $\theta>0$ so small that $\theta<b-a$ and $C_{a, b} C_{0} A_{2} \exp \left(C_{0} \theta\right) \chi_{1}(\theta) \leqq C_{5} / 8$, we obtain, by (5.7),

$$
\begin{gathered}
C_{2}\|u(a+\theta)\|_{\rho}^{2}+C_{3} \int_{a}^{a+\theta}\|u\|_{\rho+k}^{2} d t+\left(C_{5} / 8\right) \int_{a}^{a+\theta}\|u\|_{\rho}^{2} d t \\
\leqq \\
C_{a, b} C_{0} \exp \left(C_{0} \theta\right) \chi_{2}(\theta) \sum_{j=1}^{m-1}\left\|D_{t}^{j-1} \phi(a)\right\|_{\rho}^{2} \\
\quad+C_{1} \exp \left(C_{0} \theta\right)\|u(a)\|_{\rho}^{2}+C_{0} \exp \left(C_{0} \theta\right)\|f\|_{0, \rho, \Omega}^{2}
\end{gathered}
$$

for all $\phi \in \mathscr{H}^{r, s}(\Omega), u=u_{\phi}, f=P \phi$ and $\Omega=\Omega_{a, a+\theta}$. Thus, if $\phi \in \mathscr{H}^{r, s}\left(\Omega_{a, b}\right)$ and $P \phi=0$, then $\phi=0$ on $\Omega_{a, a+\theta}$. Applying the estimate (5.8) for the slab $\Omega_{a+\theta, a+2 \theta}$, we see that $\phi=0$ on $\Omega_{a+\theta, a+2 \theta}$. Repeating this argument we obtain $\phi=0$ on $\Omega_{a, b}$ and our proof is complete.

\section{The Cauchy problem for $R$.}

LEMMA 4. Let $R=\partial / \partial t-h \Lambda^{2 k}$, where $h$ is a constant $m \times m$ matrix of the form (2.1) having its eigenvalues contained in $\Delta$. Then there exists $C>0$ depending on $\Delta$, $\delta, k, m$ and $\|h\|$ such that

$$
\int_{R^{n+1}}\left\{\tau^{2}+|\xi|^{4 k}+\lambda^{2}\right\}|\hat{u}(\xi, \tau)|^{2} d \xi d t \leqq C\|(R+\lambda I) u\|_{0,0}^{2}
$$

for all $u \in\left\{C_{0}^{\infty}\left(R^{n+1}\right)\right\}^{m}$ and all $\lambda>0$. 
Proof. Let $R(\xi, \tau)=i \tau I-|\xi|^{2 k} h$; thus $R=R\left(D, D_{t}\right) . R(\xi, \tau)+\lambda I$ is invertible for all $\langle\xi, \tau\rangle \in R^{n+1}$ and $\lambda>0$; moreover, for $\xi \neq 0$

$$
\{R(\xi, \tau)+\lambda I\}^{-1}=|\xi|^{-2 k}\left\{|\xi|^{-2 k}(i \tau+h) I-h\right\}^{-1} .
$$

For fixed $\xi \in R^{n} \sim\{0\}, \tau \in R^{1}$ and $\lambda>0$, the function $z \rightarrow\left\{\lambda+i \tau-z|\xi|^{2 k}\right\}^{-1}$ is holomorphic in $\{z \in C: \operatorname{Re} z<0\}$. Thus, if $\Gamma$ is a closed contour surrounding $\Delta$ such that $\operatorname{Re} z \leqq-\delta / 2$ for all $z \in \Gamma$, then

Thus

$$
\{R(\xi, \tau)+\lambda I\}^{-1}=\frac{1}{2 \pi i} \int_{\Gamma}\left(\lambda+i \tau-z|\xi|^{2 k}\right)^{-1}(z I-h)^{-1} d z .
$$

$$
\left\|\{R(\xi, \tau)+\lambda I\}^{-1}\right\| \leqq \frac{l(\Gamma)}{2 \pi} \sup _{z \in \Gamma} \frac{\left\|(z I-h)^{-1}\right\|}{\left.|\lambda+i \tau-z| \xi\right|^{2 k} \mid}
$$

for all $\xi \in R^{n} \sim\{0\}$ and $\tau \in R^{1}$, where $l(\Gamma)$ denotes the length of $\Gamma$. We assert that there exists $C=C(\Delta, \delta)>0$ such that

$$
C\left\{|\xi|^{4 k}+\tau^{2}+\lambda^{2}\right\} \leqq \inf \left\{\left.\left.|(\lambda+i \tau)-| \xi\right|^{2 k} z\right|^{2}: z \in \Gamma\right\}
$$

for all $\langle x, \tau\rangle \in R^{n+1}$ and $\lambda>0$. For $z=x+i y \in \Gamma$ we have that $\left.\left.|\lambda-| \xi\right|^{2 k} x\right|^{2}$ $\geqq\left(\delta^{2} / 4\right)\left(\lambda^{2}+|\xi|^{4 k}\right)$. Thus

$$
\left.\left.|(\lambda+i \tau)-| \xi\right|^{2 k} z\right|^{2} \geqq\left(\delta^{2} / 4\right)\left(\lambda^{2}+|\xi|^{4 k}\right)+\left.\left.|\tau-| \xi\right|^{2 k} y\right|^{2}
$$

for all $z=x+i y \in \Gamma$. Letting $\gamma=\sup \{|\operatorname{Im} z|: z \in \Gamma\}$ it is easily shown that there exists $C=C(\gamma)>0$ such that $\left(\gamma^{2} / 2\right) x^{2}+|\tau-x y|^{2} \geqq C\left(x^{2}+y^{2}\right)$ for all $x \in[0, \infty)$, $\tau \in R^{1}, y \in[-\gamma, \gamma]$. Thus (6.2) holds. Now by choosing $\Gamma$ sufficiently close to $\Delta$, say $\delta / 8 \leqq \operatorname{dist}(\lambda, \Gamma) \leqq \delta / 4$ for each $\lambda \in \Delta$, we obtain, by (6.1),

$$
\left\|\{R(\xi, \tau)+\lambda I\}^{-1}\right\| \leqq C\left\{\tau^{2}+|\xi|^{4 k}+\lambda^{2}\right\}^{-1 / 2} \sup _{z \in \Gamma}\left\|(z I-h)^{-1}\right\|,
$$

where $C=C(\Delta, \delta)>0$. Since $h$ is of the form $(2.1),(z I-h)^{-1}$ is equal to $(\operatorname{det}(z I-h))^{-1}$ times a matrix whose entries are polynomials in $p_{1}, \ldots, p_{m}$ and $z$. If we let $\beta=\operatorname{dist}(\Delta, \Gamma)$, it follows that

$$
|\operatorname{det}(z I-h)| \geqq \beta^{m} \geqq(\delta / 8)^{m} \text { and } \sup _{z \in \Gamma}\left\|(z I-h)^{-1}\right\| \leqq C \delta^{-m}
$$

where $C>0$ depends on $\Delta, \delta, k, m$ and a polynomial in $p_{1}, \ldots, p_{m}$ whose degree depends only on $m$. Writing $\hat{u}=\{R+\lambda I\}^{-1}\{R+\lambda I\} \hat{u}$ we obtain

$$
|\hat{u}(\xi, \tau)|^{2} \leqq C|\{R(\xi, \tau)+\lambda I\} \hat{u}(\xi, \tau)|^{2}\left\{|\xi|^{4 k}+\tau^{2}+\lambda^{2}\right\}
$$

for $\lambda>0,\langle\xi, \tau\rangle \in R^{n+1}$ and our proof is complete.

LEMMA 5. Let $H(t)$ and $J(t)$ be given by (3.8) and (3.7), respectively, and suppose $R=\partial / \partial t-H(t) \Lambda^{2 k}-J(t)$. Then there exist constants $C_{1}, C_{2}>0$ depending on $P$, $\Delta, \delta, k$ and $m$ such that

$$
\int_{R^{n+1}}\left\{\tau^{2}+|\xi|^{4 k}+\lambda^{2}\right\}|\hat{u}(\xi, \tau)|^{2} d \xi d \tau \leqq C_{1}\|(R+\lambda I) u\|_{0,0}^{2}+C_{2}\|u\|_{0,0}^{2}
$$

for all $u \in\left\{C_{0}^{\infty}\left(R^{n+1}\right)\right\}^{m}$ and all $\lambda$ sufficiently large. 
Proof. We refer the reader to $\S 3$ for the definition of $\left(Q_{i}, \zeta_{i}\right),\left(\Omega_{j}, \zeta_{j}\right), h_{i j}$ and $N_{i j}, i=1,2, \ldots, j=1, \ldots, s$, in which $\left(Q_{i}, \zeta_{i}\right)_{1}^{\infty},\left(\Omega_{j}, \phi_{j}\right)_{1}^{s}$ are determined by the number $\eta$ satisfying (3.15). We define $R^{i j}=\partial / \partial t-h_{i j} \Lambda^{2 k}$ and the operator $\Phi_{j}$ by $\mathscr{F}\left(\Phi_{j} u\right)(\xi, \tau)=\phi_{j}(\xi) \hat{u}(\xi, \tau)$ for $u \in C_{0}^{\infty}\left(R^{n+1}\right)$ and extended. Since the matrices $h_{i j}$ are uniformly bounded, there exists, by Lemma $4, C=C(\Delta, \delta, k, m, p)>0$ satisfying, for each $i$ and $j$,

$$
\int_{R^{n+1}}\left\{\tau^{2}+|\xi|^{4 k}+\lambda^{2}\right\}|\hat{u}(\xi, \tau)|^{2} d \xi d \tau \leqq C\left\|\left(R^{i j}+\lambda I\right) u\right\|_{0,0}^{2}
$$

for all $u \in\left\{C_{0}^{\infty}\left(R^{n+1}\right)\right\}^{m}$ and all $\lambda>0$. Our proof shall be roughly like that of Theorem 2. Employing the above estimate (6.3) and the identity in (3.23) we shall estimate $\sum_{i, j}\left\|\left(R^{i j}+\lambda I\right)\left(\zeta_{i} \Phi_{j} u\right)\right\|_{0,0}^{2}$ in terms of $\|(R+\lambda I) u\|_{0,0}^{2}$ and various "error" terms.

Consider the following estimates on the $L^{2}$ norms of the last five terms on the right side of (3.23) applied to $u \in\left\{C_{0}^{\infty}\left(R^{n+1}\right)\right\}^{m}$ ([A,B] denotes the commutator of $A$ and $B)$ :

(1) Since $\left[\partial / \partial t, \zeta_{i}\right]=\partial \zeta_{i} / \partial t$, we apply (3.20) to obtain

$$
\sum_{i, j}\left\|\left[\partial \zeta_{i} / \partial t\right] \Phi_{j} u\right\|_{0,0}^{2} \leqq \tilde{C}(\eta)\|u\|_{0,0}^{2}
$$

(2) Since $\left\|h_{i j}\right\|$ is bounded independent of $i$ and $j$ (see Theorem 1), we obtain

$$
\begin{aligned}
\sum_{i, j}\left\|h_{i j}\left[\Lambda^{2 k}, \zeta_{i}\right] \Phi_{j} u\right\|_{0,0}^{2} & \leqq C \sum_{|\alpha|=k ; 0<\beta \leqq 2 \alpha} \sum_{i, j}\left\|\left(D^{\beta} \zeta_{i}\right) D^{2 \alpha-\beta} \Phi_{j} u\right\|_{0,0}^{2} \\
& \leqq \tilde{C}(\eta) \sum_{|\alpha|=k ; 0<\beta \leqq 2 \alpha}\left\|D^{2 \alpha-\beta} \Phi_{j} u\right\|_{0,0}^{2} \\
& \leqq \tilde{C}(\eta)\|u\|_{0,2 k-1}^{2} .
\end{aligned}
$$

(3) Since coefficients of $P_{0}$ are elements of $C_{B}^{\infty}\left(R^{n+1}\right)$, we obtain, by Proposition 1 and (3.5),

$$
\sum_{i, j}\left\|\zeta_{i}\left[\Phi_{j}, H\right] \Lambda^{2 k} u\right\|_{0,0}^{2}=\sum_{i, j}\left\|\left[\Phi_{j}, H\right] \Lambda^{2 k} u\right\|_{0,0}^{2} \leqq \widetilde{C}(\eta)\|u\|_{0,2 k-1}^{2} .
$$

(4) Since $J(t)$ is uniformly of order $2 k-1$ for $t \in R^{1}$, we have, by (3.21),

$$
\sum_{i, j}\left\|\left[\zeta_{i} \Phi_{j}, J(t)\right] u\right\|_{0,0}^{2} \leqq \widetilde{C}(\eta)\|u\|_{0,2 k-1}^{2} .
$$

(5) To estimate $\chi=\sum_{i, j}\left\|\zeta_{i}\left[H(t)-h_{i j}\right] \Lambda^{2 k} \Phi_{j} u\right\|_{0,0}^{2}$ we apply the techniques of Theorem 2; thus we write $H(t)-h_{i j}=k_{j}^{\prime}(x, t ; D)+s_{i j}(t)$. where $k_{j}^{\prime}(x, t ; \xi)$ $=h(x, t ; \xi)-h\left(x, t ; \xi_{j}\right)$ and $s_{i j}(x, t)=h\left(x, t ; \xi_{j}\right)-h\left(x_{i} ; t_{i} ; \xi_{j}\right)$. For each $j=1,2$, $\ldots, s$, let $\psi_{j} \in C_{0}^{\infty}\left(\Omega_{j}\right)$ with $\psi_{j} \equiv 1$ on the support of $\phi_{j}$ and $0 \leqq \psi_{j} \leqq 1$. Extend $\psi_{j}$ to $R^{n} \sim\{0\}$ and define $k_{j}(x, t ; \xi)=\psi_{j}(\xi) k_{j}^{\prime}(x, t ; \xi)$. Letting $K_{j}(t)=k_{j}(x, t ; D)$, we have $k_{j}^{\prime}(x, t ; D) \Phi_{j}=K_{j}(t) \Phi_{j}, j=1, \ldots, s$. Thus

$$
\chi \leqq 2 \sum_{j=1}^{s}\left\|K_{j} \Phi_{j} \Lambda^{2 k} u\right\|_{0,0}^{2}+2 \sum_{i, j=1}\left\|\zeta_{i} s_{i j} \Phi_{j} \Lambda^{2 k} u\right\|_{0,0}^{2} .
$$


To obtain an estimate on the first term on the right side of (6.5) we recall, by (3.19), that $\left\|k_{j}(x, t ; \xi)\right\|\left\langle\eta / 2\right.$ for all $\langle x, t ; \xi\rangle \in R^{n+1} \times \Sigma$. Letting $\varepsilon=\eta / 2$ in Proposition 2 and integrating with respect to $t \in R^{1}$ we obtain

$$
\sum_{j=1}^{s}\left\|K_{j} \Phi_{j} \Lambda^{2 k} u\right\|_{0,0}^{2} \leqq \eta^{2}\|u\|_{0,2 k}^{2}+\widetilde{C}(\eta)\|u\|_{0,2 k-1}^{2} .
$$

For the second term on the right side of (6.5) we apply (3.18):

$$
\sum_{i, j}\left\|\zeta_{i} s_{i j} \Phi_{j} \Lambda^{2 k} u\right\|_{0,0}^{2} \leqq \frac{\eta^{2}}{4} \sum_{i, j}\left\|\zeta_{i} \Phi_{j} \Lambda^{2 k} u\right\|_{0,0}^{2} \leqq C_{k} \eta^{2}\|u\|_{0,2 k}^{2}
$$

Thus $\chi \leqq C_{k} \eta^{2}\|u\|_{0,2 k}^{2}+\tilde{C}(\eta)\|u\|_{0,2 k-1}^{2}$.

Combining estimates (1) through (5) and applying (3.1) we obtain, for arbitrary $\varepsilon>0$,

$$
\begin{aligned}
& \sum_{i, j}\left\|\left(R^{i j}+\lambda I\right)\left(\zeta_{i} \Phi_{j} u\right)\right\|_{0,0}^{2} \\
& \leqq\|(R+\lambda I) u\|_{0,0}^{2}+\left\{C \eta^{2}+\widetilde{C}(\eta)\right\}\|u\|_{0,2 k}^{2}+\widetilde{C}(\eta) C(\varepsilon)\|u\|_{0,0}^{2}
\end{aligned}
$$

for all $u \in\left\{C_{0}^{\infty}\left(R^{n+1}\right)\right\}^{m}$. Thus, by $(6.3),(6.6)$ and the proof of Lemma 4 we obtain, for arbitrary $\varepsilon>0$,

$$
\begin{aligned}
& \int_{R^{n+1}}\left\{\tau^{2}+|\xi|^{4 k}+\lambda^{2}\right\}|\hat{u}(\xi, \tau)|^{2} d \xi d \tau-\tilde{C}_{1}(\eta)\|u\|_{0,0}^{2} \\
& \leqq C_{2}\|(R+\lambda I) u\|_{0,0}^{2}+\left\{C \eta^{2}+\tilde{C}_{3}(\eta) \varepsilon\right\}\|u\|_{0,2 k}^{2}+\widetilde{C}_{3}(\eta) C(\varepsilon)\|u\|_{0,0}^{2} .
\end{aligned}
$$

Applying (4.1) with $p=1$, we obtain $Q^{4 k}(\xi, \tau) \leqq C_{k, 1}\left\{\tau^{2}+|\xi|^{4 k}+\lambda^{2}\right\}$ for all $\lambda \leqq 1$. Since $\|u\|_{0,2 k} \leqq\|u\|_{2 k, 0}$, we can take $\eta=\left(4 C C_{k, 1}\right)^{-1 / 2}$ and $\varepsilon=\left(4 C_{k, 1} \tilde{C}_{3}(\eta)\right)^{-1}$ and our proof is complete.

REMARK. It is easily seen that $\|u\|_{2 k, 0} \leqq C\|(R+\lambda I) u\|_{0,0}$ for all $u \in\left\{C_{0}^{\infty}\left(R^{n+1}\right)\right\}^{m}$, and for all $\lambda$ sufficiently large.

TheOrem 5 (COMPARE WiTh TheOREM 2 of [5]). Given any real $r$ and $s$, for $\lambda$ real and sufficiently large (depending on $r, s$ and $R$ ) $R+\lambda I$ is a topological isomorphism of $\left\{\mathscr{H}^{r, s}\right\}^{m}$ onto $\left\{\mathscr{H}^{r-2 k, s}\right\}^{m}$.

Proof. The continuity of $R+\lambda I$ for all $\lambda$ follows from the results of $\$ \S 3$ and 4; here $I$ is the inclusion mapping of $\left\{\mathscr{H}^{r, s}\right\}^{m}$ into $\left\{\mathscr{H}^{r-2 k, s}\right\}^{m}$. We next establish the following: there exists $C=C_{r, s}>0$ such that

$$
\|u\|_{r+2 k, s} \leqq C\|(R+\lambda I) u\|_{r, s}
$$

for all $u \in\left\{C_{0}^{\infty}\left(R^{n+1}\right)\right\}^{m}$ and all $\lambda$ sufficiently large. By Lemma 6 ,

$$
\int_{R^{n+1}}\left\{\tau^{2}+|\xi|^{4 k}+\lambda^{2}\right\}\left|\mathscr{F}\left(\mathscr{M}_{r, s} u\right)(\xi, \tau)\right|^{2} d \xi d \tau \leqq C_{1}\left\|(R+\lambda I) \mathscr{M}_{r, s} u\right\|_{0,0}^{2}+C_{2}\|u\|_{r, s}^{2} .
$$


We write $(R+\lambda I) \mathscr{M}_{r, s} u=\mathscr{M}_{r, s}(R+\lambda I) u+\left[\mathscr{M}_{r, s}, H\right] \Lambda^{2 k} u+\left[\mathscr{M}_{r, s}, J\right] u$. Fixing a $\theta$ between 0 and 1, and applying Propositions 4 and 5 of [5], we obtain, with $C=C(\theta, r, s)>0$,

$$
\left\|\left(\mathscr{M}_{r, s} H-H \mathscr{M}_{r, s}\right) \Lambda^{2 k} u\right\|_{0,0} \leqq C\|u\|_{r, s+2 k-\theta} .
$$

Applying Proposition 2(ii) of [5] we obtain, for arbitrary $\varepsilon>0$,

$\left\|\left(\mathscr{M}_{r, s} H-H \mathscr{M}_{r, s}\right) \Lambda^{2 k} u\right\|_{0,0}^{2} \leqq(\varepsilon / 2)\|u\|_{r, s+2 k}^{2}+C(\varepsilon)\|u\|_{r, s}^{2} \leqq(\varepsilon / 2)\|u\|_{r+2 k, s}^{2}+C(\varepsilon)\|u\|_{r, s}^{2}$.

Similarly

$$
\left\|\left(\mathscr{M}_{r, s} J-J \mathscr{M}_{r, s}\right) u\right\|_{0,0}^{2} \leqq(\varepsilon / 2)\|u\|_{r+2 k, s}^{2}+C(\varepsilon)\|u\|_{r, s}^{2}
$$

Thus, for arbitrary $\varepsilon>0$,

$$
\begin{aligned}
\int_{R^{n+1}}\left\{\tau^{2}+|\xi|^{4 k}+\lambda^{2}\right\} Q^{2 r}(\xi & \tau) q^{2 s}(\xi)|\hat{u}(\xi, \tau)|^{2} d \xi d \tau \\
& \leqq C_{1}\|(R+\lambda I) u\|_{r, s}^{2}+\varepsilon\|u\|_{r+2 k, s}^{2}+C(\varepsilon)\|u\|_{r, s}^{2}
\end{aligned}
$$

By (4.1) we have that $Q^{4 k}(\xi, \tau) \leqq C_{k, 1}\left\{\tau^{2}+|\xi|^{4 k}+1\right\}$. Letting $\varepsilon=1 / 2 C_{k, 1}$ and $\lambda \geqq\left\{1+2 C_{k}\right\}^{1 / 2}$ completes the proof of $(6.8)$.

If $R^{*}$ is the formal adjoint of $R$, i.e., $R^{*}=-\partial / \partial t-\Lambda^{2 k} H^{*}-J^{*}$, then we assert that for every real $r$ and $s$ there exists $C=C_{r, s}>0$ such that

$$
\|u\|_{r+2 k, s} \leqq C\left\|\left(R^{*}+\lambda I\right) u\right\|_{r, s}
$$

for all $u \in\left\{C_{0}^{\infty}\left(R^{n+1}\right)\right\}^{m}$ and all $\lambda$ sufficiently large. We write $R^{\#}=R_{1}+R_{2}$ where $R_{1}=-\partial / \partial t-H^{\#} \Lambda^{2 k}-J$ and $R_{2}=H^{\#} \Lambda^{2 k}-\Lambda^{2 k} H^{\#}$. By Theorem 3 no. ${ }^{\circ} 7$ of [7], we have that for any real $s$

$$
\begin{aligned}
\left\|H(t)^{\#} \Lambda^{2 k}-\Lambda^{2 k} H(t)^{\#}\right\|_{H^{s} \rightarrow H^{s-(2 k-1)}} & \leqq C\left\|H(t)^{\#} \Lambda-\Lambda H(t)^{\#}\right\|_{H^{3} \rightarrow H^{s}} \\
& \leqq C_{s}\|h(t)\|_{[|m|+3,2 n+3]}
\end{aligned}
$$

for all $t \in R^{1}, m=[s]$ (here $\|A\|_{X \rightarrow Y}$ denotes the operator norm of the bounded linear mapping $A$ ). Thus, by (3.5), we see that $H^{\#} \Lambda^{2 k}-\Lambda^{2 k} H^{\#}$ is uniformly of order $2 k-1$ for $t \in R^{1}$. Applying (6.9) (with $R$ replaced by $R_{1}$ ), (3.2) and the inequality $\|u\|_{0,2 k} \leqq\|u\|_{2 k, 0}$, we obtain, for arbitrary $\varepsilon>0$,

$$
\begin{aligned}
& \int_{R^{n+1}}\left\{\tau^{2}+|\xi|^{4 k}+\lambda^{2}\right\}\left|\mathscr{F}\left(\mathscr{M}_{r, s} u\right)(\xi, \tau)\right|^{2} d \xi d \tau \\
& \leqq C_{1}\left\|\left(R_{1}+\lambda I\right) \mathscr{M}_{r, s} u\right\|_{0,0}^{2}+C_{2}\|u\|_{r, s}^{2} \\
& \leqq C_{1}\left\|\left(R^{\#}+\lambda I\right) \mathscr{M}_{r, s} u\right\|_{0,0}^{2}+C_{1}\left\|\left(H^{\#} \Lambda^{2 k}-\Lambda^{2 k} H^{\#}\right) \mathscr{M}_{r, s} u\right\|_{0,0}^{2}+C_{2}\|u\|_{r, s}^{2} \\
& \leqq C_{1}\left\|\left(R^{\#}+\lambda I\right) u\right\|_{r, s}^{2}+\varepsilon\|u\|_{r, s}^{2}+C(\varepsilon)\|u\|_{r, s}^{2} .
\end{aligned}
$$

We write

and

$$
\mathscr{M}_{r, s}\left(R^{\#}+\lambda I\right)=\mathscr{M}_{r, s}\left(R^{*}+\lambda I\right)+\mathscr{M}_{r, s} \Lambda^{2 k}\left(H^{*}-H^{\#}\right)+\mathscr{M}_{r, s}\left(J^{*}-J^{\#}\right),
$$

$$
\mathscr{M}_{r, s} \Lambda^{2 k}\left(H^{*}-H^{\#}\right)=\Lambda^{2 k}\left(H^{*}-H^{\#}\right) \mathscr{M}_{r, s}+\Lambda^{2 k}\left[\mathscr{M}_{r, s}, H^{*}\right]+\Lambda^{2 k}\left[H^{\#}, \mathscr{M}_{r, s}\right] .
$$


Consider the following estimates:

(1) Applying Proposition 1, (3.5) and Propositions 2(i) and 2(ii) of [5], we obtain, for arbitrary $\varepsilon>0$,

$$
\begin{aligned}
\left\|\Lambda^{2 k}\left(H^{*}-H^{\#}\right) \mathscr{M}_{r, s} u\right\|_{0,0}^{2} & \leqq C_{k}\left\|\mathscr{M}_{r, s} u\right\|_{0,2 k-1}^{2} \\
& =C_{k}\|u\|_{r, s+2 k-1}^{2} \leqq \varepsilon\|u\|_{r+2 k, s}^{2}+C(\varepsilon)\|u\|_{r, s}^{2} .
\end{aligned}
$$

(2) Fixing a $\theta$ between 0 and 1, and applying Propositions 2(i), 2(ii) and 5(ii) of [5], we obtain for arbitrary $\varepsilon>0$,

$$
\left\|\Lambda^{2 k}\left(H^{\#} \mathscr{M}_{r, s}-\mathscr{M}_{r, s} H^{\#}\right) u\right\|_{0,0}^{2} \leqq C\|u\|_{r, s+2 k-\theta}^{2} \leqq \varepsilon\|u\|_{r+2 k, s}^{2}+C(\varepsilon)\|u\|_{r, s}^{2} .
$$

(3) We observe that $\Lambda^{2 k}\left(\mathscr{M}_{r, s} H^{*}-H^{*} \mathscr{M}_{r, s}\right)=\Lambda^{2 k}\left(H \mathscr{M}_{r, s}-\mathscr{M}_{r, s} H\right)^{*}$. Fixing a $\theta$ between 0 and 1 we have, by Proposition 5(ii) of [5], that $H \mathscr{M}_{r, s}-\mathscr{M}_{r, s} H$ is continuous from $\mathscr{H}^{0,0}$ into $\mathscr{H}^{-r,-s+\theta}$ which implies that $\mathscr{M}_{r, s} H^{*}-H^{*} \mathscr{M}_{r, s}$ is continuous from $\mathscr{H}^{r, s-\theta}$ into $\mathscr{H}^{0,0}$. Applying Proposition 2(ii) of [5], we obtain, for arbitrary $\varepsilon>0$,

$$
\left\|\Lambda^{2 k}\left(\mathscr{M}_{r, s} H^{*}-H^{*} \mathscr{M}_{r, s}\right) u\right\|_{0,0}^{2} \leqq C\|u\|_{r, s+2 k-\theta}^{2} \leqq \varepsilon\|u\|_{r+2 k, s}^{2}+C(\varepsilon)\|u\|_{r, s}^{2} .
$$

Similar estimates for $\mathscr{M}_{r, s}\left(J^{*}-J^{\#}\right)$ yield, for arbitrary $\varepsilon>0$,

$$
\begin{aligned}
& \int_{R^{n+1}}\left\{\tau^{2}+|\xi|^{4 k}+\lambda^{2}\right\} Q^{2 r}(\xi, \tau) q^{2 s}(\xi)|\hat{u}(\xi, \tau)|^{2} d \xi d \tau \\
& \leqq C_{1}\left\|\left(R^{*}+\lambda I\right) u\right\|_{r, s}^{2}+\varepsilon\|u\|_{r, s}^{2}+C(\varepsilon)\|u\|_{r, s}^{2} .
\end{aligned}
$$

As previously shown, we need only take $\varepsilon$ sufficiently small and the proof of (6.10) is complete.

As a consequence of (6.8), we conclude that the range of $R+\lambda I$ is closed in $\left\{\mathscr{H}^{r-2 k, s\}^{m}}\right.$; thus all we need show is that the range of $R+\lambda I$, for $\lambda$ sufficiently large, has trivial orthogonal complement in $\left\{\mathscr{H}^{r-2 k, s}\right\}^{m}$. Now suppose $v \in\left\{\mathscr{H}^{r-2 k, s}\right\}^{m}$ is such that for all $u \in\left\{C_{0}^{\infty}\left(R^{n+1}\right)\right\}^{m}, 0=((R+\lambda I) u, v)_{r, s}=\left[(R+\lambda I) u, \mathscr{M}_{2 r, 2 s} v\right]$. Then $\omega=\mathscr{M}_{2 r, 2 s} v \in\left\{\mathscr{H}^{-r-2 k,-s}\right\}^{m}$ and $\left(R^{*}+\lambda I\right) \omega=0$, which, by (6.10) applied to $\omega$, with $-r-2 k$ and $-s$ replacing $r$ and $s$, implies that $\omega=0$ and therefore $v=0$.

For any closed set $K \subseteq R^{n+1}$, we set $\mathscr{H}_{0}^{r, s}(K)=\left\{u \in \mathscr{H}^{r, s}:\right.$ supp $\left.u \subseteq K\right\}$. Letting a bar denote closure and writing $\mathscr{C} A$ for the complement of $A$, we have that $C_{0}^{\infty}(\mathscr{C} \bar{\Omega})$ is dense in $\mathscr{H}_{0}^{r, s}\left(\mathscr{C} \Omega\right.$ ) for all real $r$ and $s$, with $\Omega=\Omega_{a, b},-\infty \leqq a<b \leqq+\infty$ (see $\S 4$ of [5]).

TheOREM 6 (COMPARE With THeOReM 4 OF [5]). Given any real $r, s$ and $c$, $R+\lambda I$ is an isomorphism of $\left\{\mathscr{H}_{0}^{r, s}\left(\bar{\Omega}_{c,+\infty}\right)\right\}^{m}$ onto $\left\{\mathscr{H}_{0}^{r-2 k, s}\left(\bar{\Omega}_{c,+\infty}\right)\right\}^{m}$ for all $\lambda$ sufficiently large (depending on $r, s$ and $R$ ).

Proof. First choose $\rho_{0}>k, \rho_{0}>r$. Next, choose $\lambda_{0}$ so large that

(i) $\lambda_{0} \geqq C_{4}\left(C_{4}\right.$, the constant in Theorem 3$)$, and

(ii) $\lambda \geqq \lambda_{0}$ implies that $R+\lambda I$ is an isomorphism of $\left\{\mathscr{H}^{r, s}\right\}^{m}$ onto $\left\{\mathscr{H}^{r-2 k, s}\right\}^{m}$ and $\left\{\mathscr{H}^{\rho_{0}, s}\right\}^{m}$ onto $\left\{\mathscr{H}^{\rho_{0}-2 k, s}\right\}^{m}$ (applying Theorem 5 in both cases). 
We assert that for $\lambda \geqq \lambda_{0}, R+\lambda I$ has the desired property. By Theorem 5 it is clear that we need only show that if $u \in\left\{\mathscr{H}^{r, s}\right\}^{m}$ and $v=(R+\lambda I) u$ has its support in $\bar{\Omega}_{c,+\infty}$, then so does $u$. Since $\left\{C_{0}^{\infty}\left(\Omega_{c,+\infty}\right)\right\}^{m}$ is dense in $\left\{\mathscr{H}_{0}^{r-2 k, s}\left(\Omega_{c,+\infty}\right)\right\}^{m}$, then there exists a sequence $\left\{v_{j}\right\}$ in $\left\{C_{0}^{\infty}\left(\Omega_{c,+\infty}\right)\right\}^{m}$ converging to $v$ in $\left\{\mathscr{H}^{r-2 k, s}\right\}^{m}$. By Theorem 5, for each $j$ there exists a unique $u_{j} \in\left\{\mathscr{H}^{r, s}\right\}^{m}$ satisfying $(R+\lambda I) u_{j}=v_{j}$; however, there also exists $u_{j}^{\prime} \in\left\{\mathscr{H}^{\rho_{0}}, s\right\}^{m}$ such that $(R+\lambda I) u_{j}^{\prime}=v_{j}$. Thus $u_{j}=u_{j}^{\prime}$ $\in\left\{\mathscr{H}^{\rho_{0}}, s\right\}^{m}$; applying Theorem 3 with $a=-\infty$ and arbitrary $b<c$ we see that $u_{j}(b)=0$. Thus each $u_{j}$ has its support in $\bar{\Omega}_{c,+\infty}$, and the same remains true for $u$.

TheOREM 7 (COMPARE With TheOREM 5 OF [5]). If $s$ is real, $r>k$, and $-\infty<a<b<+\infty$, the mapping $u \leadsto\langle R u, u(a)\rangle$ is a topological isomorphism of $\left\{\mathscr{H}^{r, s}(\Omega)\right\}^{m}$ onto $\left\{\mathscr{H}^{r-2 k, s}(\Omega)\right\}^{m} \oplus\left\{H^{r-k+s}\right\}^{m}$, where $\Omega=\Omega_{a, b}$.

Proof. With only slight modifications the proof is exactly like that of Theorem 5 of [5] with $R$ replacing $P$, our form of the energy inequality replacing Theorem 3 of [5], and our Theorem 6 replacing Theorem 4 of [5].

\section{Existence in the Cauchy problem for $P$.}

Notation. (i) We assume that $-\infty<a<b<+\infty$. Let $r>k$; for $\theta \in[a, b]$ we let $E_{\theta}$ be the evaluation map $u \rightarrow u(\theta)$ of $\mathscr{H}^{r, s}(\Omega)$ into $H^{r+s-k}$, where $\Omega=\Omega_{a, b}$.

(ii) For $r$ and $s$ real, $r>(2 m-1) k$ we define the Banach space $H \equiv H^{r+s-k}$ $\oplus H^{r+s-3 k} \oplus \cdots \oplus H^{r+s-(2 m-1) k}$. Let

$$
\mathscr{L} \phi=\left\langle\left(P_{0}+P_{1}\right) \phi ; \phi(a),(\partial / \partial t) \phi(a), \ldots,(\partial / \partial t)^{m-1} \phi(a)\right\rangle .
$$

(iii) If $X$ and $Y$ are Banach spaces and $T$ is a bounded linear mapping of $X$ into $Y$, we denote the operator norm of $T$ by

$$
\|T\|_{X \rightarrow Y}=\sup \left\{\|T x\|_{Y}:\|x\|_{X} \leqq 1\right\} .
$$

Lemma 6. Let $r$, $s$ and $\sigma$ be real, $r>k$. If $u \in \mathscr{H}^{r, s}$ and $\operatorname{supp} \Lambda^{2 \sigma} u \subset \mathscr{C} \Omega$ then supp $u \subset \mathscr{C} \Omega$.

Proof. Since $\Lambda^{2 \sigma}\left(E_{t} u\right)=E_{t}\left(\Lambda^{2 \sigma} u\right)=0$ for all $t \in[a, b]$ we see that $E_{t}=0$ for all $t \in[a, b]$. Thus by Proposition 9 of $[5], u \mid \Omega=0$.

THEOREM 8. If $r$ and $s$ are real numbers with $r>(2 m-1) k$, then $\mathscr{L}$ is a topological isomorphism of $\mathscr{H}^{r, s}(\Omega)$ onto $\mathscr{H}^{r-2 k m, s}(\Omega) \oplus H$, where $\Omega=\Omega_{a, b}$.

Proof. By Theorem 4 we have that $\mathscr{L}$ is a one-to-one mapping. By the open mapping principle it remains only to show that $\mathscr{L}$ is onto. Let $\left\langle f, \phi, \ldots, \phi_{m}\right\rangle$ $\in \mathscr{H}^{r-2 k m, s}(\Omega) \oplus H$ where $\phi_{j} \in H^{r+s-(2 j+1) k}, j=0,1, \ldots, m-1$. We must find $\phi \in \mathscr{H}^{r, s}(\Omega)$ satisfying

$$
\left(P_{0}+P_{1}\right) \phi=f, \quad(\partial / \partial t)^{j-1} \phi(a)=\phi_{j}, \quad j=1,2, \ldots, m .
$$

We recall, by Theorem 7, that $\mathscr{R}=\left\langle\partial / \partial t-H(t) \Lambda^{2 k}-J(t) ; E_{a}\right\rangle$ is a topological isomorphism of $\left\{\mathscr{H}^{r-2 k(m-1), s}(\Omega)\right\}^{m}$ onto $\left\{\mathscr{H}^{r-2 k m, s}(\Omega)\right\}^{m} \oplus\left\{H^{[r-2 k(m-1)]+s-k}\right\}^{m}$. If 
$\phi \in \mathscr{H}^{r, s}(\Omega)$ and if we define $u_{\phi}=u=\left\{u_{j}\right\}_{1}^{m} \in\left\{\mathscr{H}^{r-2 k(m-1), s}(\Omega)\right\}^{m}$ by $u_{j}=\Lambda^{2 k(m-j)} D_{t}^{j-1} \phi$, $j=1, \ldots, m$, then $\mathscr{R} u=\left\langle i\left(P_{0}+P_{1}\right) \varepsilon_{m},\left\{u_{j}(a)\right\}_{1}^{m}\right\rangle$. In particular if $\phi$ satisfies (7.1) then $\mathscr{R} u=\left\langle(i f) \varepsilon_{m},\left\{i^{-(j-1)} \Lambda^{2 k(m-j)} \phi_{j}\right\}_{1}^{m}\right\rangle$. Let

$$
U=\mathscr{R}^{-1}\left\langle(\text { if }) \varepsilon_{m},\left\{i^{-(j-1)} \Lambda^{2 k(m-j)} \phi_{j}\right\}_{1}^{m}\right\rangle .
$$

It suffices to find $\phi \in \mathscr{H}^{r, s}(\Omega)$ such that $u_{\phi}=U$, that is, such that $\Lambda^{2 k(m-j)} D_{t}^{j-1} \phi$ $=U_{j}, j=1, \ldots, m$. For in that case $\mathscr{R} u_{\phi}=\mathscr{R} U$ which implies $\left(P_{0}+P_{1}\right) \phi=f$ and $\Lambda^{2 k(m-j)} \phi_{j}=\Lambda^{2 k(m-j)}(\partial / \partial t)^{j-1} \phi(a), j=1, \ldots, m$. Thus $(\partial / \partial t)^{j-1} \phi(a)=\phi_{j}, j=1, \ldots, m$.

Assuming $m \geqq 2$ we use an induction argument on Theorem 9 of [5] to see that the mapping

$$
\mathscr{T} \phi=\left\langle\left(\partial / \partial t+\Lambda^{2 k}\right)^{m-1} \phi, \phi(a),(\partial / \partial t) \phi(a), \ldots,(\partial / \partial t)^{m-2} \phi(a)\right\rangle
$$

is a topological isomorphism of $\mathscr{H}^{r, s}(\Omega)$ onto $\mathscr{H}^{r-2 k(m-1), s}(\Omega) \oplus H^{r+s-k} \oplus \cdots$ $\oplus H^{r+s-(2 m-3) k}$. Thus there exists a unique element $\phi \in \mathscr{H}^{r, s}(\Omega)$ satisfying

$$
\begin{aligned}
\left(\partial / \partial t+\Lambda^{2 k}\right)^{m-1} \phi & =\sum_{j=0}^{m-1}\left(\begin{array}{c}
m-1 \\
j
\end{array}\right) i^{(m-j-1)} U_{m-j}, \\
(\partial / \partial t)^{j-1} \phi(a) & =\phi_{j}, \quad j=1,2, \ldots, m-1 .
\end{aligned}
$$

We assert that $\Lambda^{2 k(m-1)} \phi=U_{1}$. By (7.2)

$$
\partial U_{j} / \partial t=i \Lambda^{2 k} U_{j+1}, \quad j=1, \ldots, m-1 .
$$

Employing (7.3) and (7.4) we obtain, by an easy calculation, $\left(\partial / \partial t+\Lambda^{2 k}\right)^{m-1} \Lambda^{2 k(m-1)} \phi$ $=\left(\partial / \partial t+\Lambda^{2 k}\right)^{m-1} U_{1}$, and $(\partial / \partial t)^{j-1} \Lambda^{2 k(m-1)} \phi(a)=(\partial / \partial t)^{j-1} U_{1}(a), j=1, \ldots, m-1$. Thus by the one-to-one property of $\mathscr{T}$ our assertion holds.

We now assert that if $\Lambda^{2 k(m-j)}(\partial / \partial t)^{j-1} \phi=i^{(j-1)} U_{j}$, then $\Lambda^{2 k(m-j-1)}(\partial / \partial t)^{j} \phi$ $=i^{(j)} U_{j+1}, j=1, \ldots, m-1$. Applying $\Lambda^{2 k}$ to both sides of the above equations yields the truth of our assertion. Thus $U=u_{\phi}$ and our theorem is proven.

Let $\mid \Omega$ denote the operation of restriction, that is, $(\mid \Omega) u=u \mid \Omega$.

Proposition 8. Let $\Omega_{1}=\Omega_{a, b}$ and $\Omega_{2}=\Omega_{a, a+\theta}$ where $\theta \in(0, b-a)$. Let $\mathscr{L}_{1}$ and $\mathscr{L}_{2}$ be the restrictions of $\mathscr{L}$ to $\mathscr{H}^{r, s}\left(\Omega_{1}\right)$ and $\mathscr{H}^{r, s}\left(\Omega_{2}\right)$, respectively, where r and $s$ are real numbers with $r>(2 m-1) k$. Then the following diagram commutes:

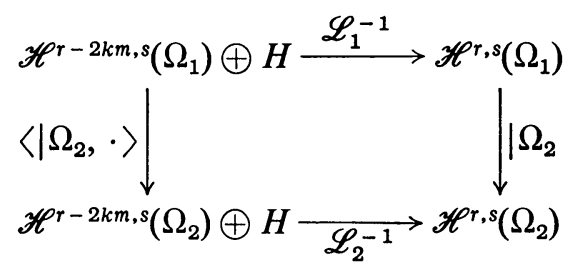

Proof. This is a trivial consequence of the one-to-one property of $\mathscr{L}_{2}$ on $\mathscr{H}^{r, s}\left(\Omega_{2}\right)$. 
COROLlARY. The operator norm of $\mathscr{L}_{2}^{-1}$, as bounded mapping from $\mathscr{H}^{r-2 k m, s}\left(\Omega_{2}\right)$ into $\mathscr{H}^{r, s}\left(\Omega_{2}\right)$, is less than or equal to the operator norm of $\mathscr{L}_{1}^{-1}$ mapping $\mathscr{H}^{r-2 k m, s}\left(\Omega_{1}\right)$ continuously into $\mathscr{H}^{r, s}\left(\Omega_{1}\right)$.

Proposition 9. Let $s$ be any real number and $r$ any nonnegative real number which is not an odd multiple of $k$. Then there exists $C=C(r)>0$ such that $\|u\|_{r-2 k, s, \Omega} \leqq C \theta\|u\|_{r, s, \Omega}$ for all $u \in \mathscr{H}^{r, s}(\Omega)$ where $\Omega=\Omega_{a, a+\theta}$ and $\theta \in(0, b-a)$.

Proof. Since $\mathscr{M}_{0, \rho}$ is an isometric isomorphism of $\mathscr{H}^{r, s}(\Omega)$ onto $\mathscr{H}^{r, s-\rho}(\Omega)$ for all $r, s, \rho$ and $\Omega$ (see $\S 4$ of [5]), we may assume without loss of generality that $s=0$. Then $\|u(t)\|_{0} \leqq\|u(t)\|_{r-k} \leqq C_{r}\|u\|_{r, 0, \Omega}$ for all $t \in[a, a+\theta]$ and $u \in C_{(0)}^{\infty}(\bar{\Omega})$ (by Proposition 9 of [5]). Thus

$$
\|u\|_{0,0, \Omega}^{2} \leqq \int_{a}^{a+\theta}\|u(t)\|_{0}^{2} d t \leqq C_{r} \theta\|u\|_{r, 0, \Omega}^{2}
$$

and

$$
\|u\|_{r-2 k, 0, \Omega} \leqq C \theta\|u\|_{r, 0, \Omega} \text { for all } u \in \mathscr{H}^{r, s}(\Omega)
$$

(assuming $0<\theta<1$ ).

We remark that in general $\mathscr{H}^{r, s}(\Omega)$ and $\mathscr{H}_{0}^{-r,-s}(\bar{\Omega})$ are dual Hilbert spaces with respect to an extension of the sesquilinear form $[u, v]_{\Omega}=\int_{\Omega} u(x, t) \bar{v}(x, t) d x d t$, $u \in C_{(0)}^{\infty}(\bar{\Omega}), \quad v \in C_{0}^{\infty}(\Omega)$. Clearly the operator norm of the inclusion map $i: \mathscr{H}_{0}^{r, 0}(\bar{\Omega}) \rightarrow \mathscr{H}_{0}^{r-2 k, 0}(\bar{\Omega})$ is bounded by $C_{r} \theta$. Since the dual map of $i$ is the inclusion $i^{*}: \mathscr{H}^{2 k-r, 0}(\Omega) \rightarrow \mathscr{H}^{-r, 0}(\Omega)$, the operator norm of $i^{*}$ is also bounded by $C_{r} \theta$. Thus the operator norm of the inclusion map $i: \mathscr{H}^{r, 0}(\Omega) \rightarrow \mathscr{H}^{r-2 k, 0}(\Omega)$ is bounded by $C_{r} \theta$ where $r \in[0, k) \cup(k, 2 k]$.

Note that $r$ can be written in the form $r=2 k j+\lambda$ where $j=1,2, \ldots$, and $\lambda \in(-k, k)$. We shall now prove the remainder of the proposition by induction on $j$. For $j=1$, we distinguish two cases: (a) For $\lambda \in(-k, 0]$ we have already shown the result. (b) For $\lambda \in(0, k)$ we observe that $r-2 k \in[0, k)$ which implies, by our previous results and Proposition 2(i) of [5],

$$
\|u\|_{r-4 k, 2 k, \Omega}=\left\|\mathscr{M}_{0,2 k} u\right\|_{r-4 k, 0, \Omega} \leqq C_{r} \theta\left\|\mathscr{M}_{0,2 k} u\right\|_{r-2 k, 0, \Omega}=C_{r} \theta\|u\|_{r, 0, \Omega}
$$

for all $u \in \mathscr{H}^{r, 0}(\Omega)$. Also

$$
\left\|\frac{\partial u}{\partial t}\right\|_{r-4 k, 0, \Omega} \leqq C_{r} \theta\left\|\frac{\partial u}{\partial t}\right\|_{r-2 k, 0, \Omega} \leqq C_{r} \theta\|u\|_{r, 0, \Omega}
$$

for all $u \in \mathscr{H}^{r, 0}(\Omega)$. Thus by Proposition 8 of [5]

$$
\|u\|_{r-2 k, 0, \Omega} \leqq\|u\|_{r-4 k, 2 k, \Omega}+\left\|\frac{\partial u}{\partial t}\right\|_{r-4 k, 0, \Omega} \leqq C_{r} \theta\|u\|_{r, 0, \Omega}
$$

for all $u \in \mathscr{H}^{r, 0}(\Omega)$. 
Suppose now our assertion is true for $j=1,2, \ldots, l-1$, where $l>1$, and $u \in \mathscr{H}^{2 k l+\lambda, 0}(\Omega)$, with $\lambda \in(-k, k)$. Then

$$
\begin{aligned}
\|u\|_{2 k(l-2)+\lambda, 2 k, \Omega} & =\left\|\mathscr{M}_{0,2 k} u\right\|_{2 k(l-2)+\lambda, 0, \Omega} \\
& \leqq C_{r} \theta\left\|\mathscr{M}_{0,2 k} u\right\|_{2 k(l-1)+\lambda, 0, \Omega}=C_{r} \theta\|u\|_{2 k l+\lambda, 0, \Omega}
\end{aligned}
$$

and

$$
\left\|\frac{\partial u}{\partial t}\right\|_{2 k(l-2)+\lambda, 0, \Omega} \leqq C_{r} \theta\left\|\frac{\partial u}{\partial t}\right\|_{2 k(l-1)+\lambda, 0, \Omega} \leqq C_{r} \theta\|u\|_{2 k l+\lambda, 0, \Omega}
$$

which was to be shown (see Proposition 8 of [5]).

REMARK. Since $Q$ (see the definition of $P$ ) is a bounded mapping of $\mathscr{H}^{r, s}\left(\Omega_{a, a+\theta}\right)$ into $\mathscr{H}^{r-2 k(m-1), s}\left(\Omega_{a, a+\theta}\right)$, its operator norm, as such a mapping, is bounded by the product of $C_{r, s}(>0)$ and the operator norm of the inclusion mapping $i: \mathscr{H}^{r-2 k(m-1), s}\left(\Omega_{a, a+\theta}\right) \rightarrow \mathscr{H}^{r-2 k m, s}\left(\Omega_{a, a+\theta}\right)$. Thus by Proposition 9, the operator norm of $Q$ as a bounded mapping of $\mathscr{H}^{r, s}\left(\Omega_{a, a+\theta}\right)$ into $\mathscr{H}^{r-2 k m, s}\left(\Omega_{a, a+\theta}\right)$ is bounded by $C_{r, s} \theta$.

By $Q \oplus 0 \oplus 0 \oplus \cdots \oplus 0$ ( $m$ zeros) we mean the mapping $u \rightarrow\langle Q u ; 0,0, \ldots, 0\rangle$ of $\mathscr{H}^{r, s}(\Omega)$ into $\mathscr{H}^{r-2 k m, s}(\Omega) \oplus H$. By Proposition 8 and the above remarks we have that

$$
\begin{aligned}
\left\|\mathscr{L}^{-1}(Q \oplus 0 \oplus \cdots \oplus 0)\right\|_{\mathscr{H}^{r, s}\left(\Omega_{a, a}+\theta\right) \rightarrow \mathscr{H}^{r, s}\left(\Omega_{a, a+\theta}\right)} & \leqq C_{r, s}\left\|\mathscr{L}^{-1}\right\|_{\mathscr{H}^{r-2 k m, s}\left(\Omega_{a, b}\right) \oplus H \rightarrow \mathscr{H}^{r, s}\left(\Omega_{a, b}\right)} \cdot \theta .
\end{aligned}
$$

However $\mathscr{L}^{-1}(Q \oplus 0 \oplus \cdots \oplus 0)=\mathscr{L}^{-1} \mathscr{P}-I$, where $I$ is the identity map on $\mathscr{H}^{r, s}\left(\Omega_{a, a+\theta}\right)$. If we choose

$$
\theta_{0}=\left\{2 C_{r, s}\left\|\mathscr{L}^{-1}\right\|_{\mathscr{H}^{r-2 k m, s}\left(\Omega_{a, b}\right) \oplus H \rightarrow \mathscr{H}^{r, s}\left(\Omega_{a, b}\right)}\right\}^{-1}
$$

then $\mathscr{L}^{-1} \mathscr{P}$ has a bounded inverse on $\mathscr{H}^{r, s}\left(\Omega_{a, a+\theta}\right)$ for all $\theta \in\left(0, \theta_{0}\right)$. Thus $\mathscr{P}$ has a bounded inverse from $\mathscr{H}^{r-2 k m, s}\left(\Omega_{a, a+\theta}\right) \oplus H$ into $\mathscr{H}^{r, s}\left(\Omega_{a, a+\theta}\right)$ for any $\theta \in\left(0, \theta_{0}\right)$; we conclude with

THEOREM 9. If $-\infty<a<b<+\infty$, $s$ is any real number and $r$ is any real number greater than $(2 m-1) k$ which is not an odd multiple of $k$, the mapping

$$
\phi \rightsquigarrow\left\langle P \phi, \phi(a),(\partial / \partial t) \phi(a),(\partial / \partial t)^{2} \phi(a), \ldots,(\partial / \partial t)^{m-1} \phi(a)\right\rangle
$$

of $\mathscr{H}^{r, s}(\Omega)$ into $\mathscr{H}^{r-2 k m, s}(\Omega) \oplus H^{r+s-k} \oplus H^{r+s-3 k} \oplus \cdots \oplus H^{r+s-(2 m-1) k}$ has a bounded inverse.

\section{Some technical details.}

Proof of Lemma 1. For $m=1$ there is nothing to show. For $m \geqq 2$ we define the numbers $\rho_{0}, \rho_{1}, \ldots, \rho_{m}$ by $\rho_{0}=1, \rho_{j}=\tau\left(\rho_{j-1}\right), j=1, \ldots, m$. Let $\varepsilon=\rho_{m}$. We define a finite sequence of subsets of $\Lambda, A_{j}, j=1, \ldots, k$, where $1 \leqq k \leqq m$, as follows. Choose any $\lambda_{1} \in \Lambda$. Let $A_{1}=\left\{\lambda \in \Lambda:\left|\lambda-\lambda_{1}\right| \geqq \rho_{1}\right\}$. If $A_{1}$ is empty, our proof is complete, since $\rho_{1}=\theta$ and (1) holds. If $A_{1}$ is nonempty, we choose $\lambda_{2} \in A_{1}$, and define 
$A_{2}=\left\{\lambda \in \Lambda: \min \left(\left|\lambda-\lambda_{1}\right|,\left|\lambda-\lambda_{2}\right|\right) \geqq \rho_{2}\right\}$. If $A_{2}$ is empty, we see that (2) holds with $k=2$, since $\rho=\left|\lambda_{1}-\lambda_{2}\right| \geqq \rho_{1} \Rightarrow \tau(\rho) \geqq \tau\left(\rho_{1}\right)=\rho_{2}$, and given any $\lambda \in \Lambda$, we must have either $\left|\lambda-\lambda_{1}\right|<\rho_{2}$ or $\left|\lambda-\lambda_{2}\right|<\rho_{2}$. If $A_{2}$ is nonempty, we choose $\lambda_{3} \in A_{2}$, and define $A_{3}=\left\{\lambda \in \Lambda: \min _{1 \leqq j \leqq 3}\left|\lambda-\lambda_{j}\right| \geqq \rho_{3}\right\}$. If $A_{3}$ is empty, we see that (2) holds with $k=3$, since $\left|\lambda_{1}-\lambda_{2}\right| \geqq \rho_{1}>\rho_{2} \Rightarrow \rho=\min _{1 \leqq i<j \leqq 3}\left|\lambda_{i}-\lambda_{j}\right| \geqq \rho_{2} \Rightarrow \tau(\rho) \geqq \tau\left(\rho_{2}\right)$ $=\rho_{3}$, and given any $\lambda \in \Lambda$, we must have either $\left|\lambda-\lambda_{1}\right|<\rho_{3},\left|\lambda-\lambda_{2}\right|<\rho_{3}$, or $\left|\lambda-\lambda_{3}\right|$ $<\rho_{3}$. If $A_{3}$ is nonempty, we define $A_{4}$ in the obvious way. Assume $A_{1}$ is nonempty; we have, after some $k$ steps, $1<k \leqq m$, that $A_{k-1}$ is nonempty and $A_{k}$ empty. Since $A_{1}, \ldots, A_{k-1}$ are nonempty, we have points $\lambda_{1}, \ldots, \lambda_{k}$ in $\Lambda$ such that

$$
\rho=\min \left\{\left|\lambda_{i}-\lambda_{j}\right|: 1 \leqq i<j \leqq k\right\} \geqq \rho_{k-1} \Rightarrow \tau(\rho) \geqq \tau\left(\rho_{k-1}\right)=\rho_{k} .
$$

Since $A_{k}$ is empty, if $\lambda \in \Lambda$ we must have either $\left|\lambda-\lambda_{1}\right|<\rho_{k}$, or $\left|\lambda-\lambda_{2}\right|<\rho_{k}, \ldots$, or $\left|\lambda-\lambda_{k}\right|<\rho_{k}$, and our proof is complete.

Lemma (COMPARE With Lemma 6.1 OF [6]). Let $a(x, \xi)$ be a symbol on $R^{n} \times \Sigma$, let $A=a(x, D)$ and suppose $\operatorname{Re} a(x, \xi) \geqq \lambda_{0}$ for all $\langle x, \xi\rangle \in R^{n} \times \Sigma$. Then for any $\varepsilon>0$ there exists $C(\varepsilon)>0$ such that $\operatorname{Re}(A \phi, \phi)_{0} \geqq\left(\lambda_{0}-\varepsilon\right)\|\phi\|_{0}^{2}-C(\varepsilon)\|\phi\|_{-1}^{2}$ for all $\phi \in C_{0}^{\infty}\left(R^{n}\right)$.

Proof. Let $b(x, \xi)=\operatorname{Re} a(x, \xi)-\lambda_{0}+\varepsilon / 2 \geqq \varepsilon / 2$ and let $g(x, \xi)=\{b(x, \xi)\}^{1 / 2}$. Clearly $b(x, \xi)$ and $g(x, \xi)$ are symbols on $R^{n} \times \Sigma$, we let $B=b(x, D)$ and $G=g(x, D)$. Since $B-G^{*} G$ is of order -1 we obtain

$$
\operatorname{Re}\left\{(B \phi, \phi)_{0}-\left(G^{*} G \phi, \phi\right)_{0}\right\} \geqq-C_{1}\|\phi\|_{-1}\|\phi\|_{0}
$$

which implies that

$$
\operatorname{Re}(B \phi, \phi)_{0} \geqq-C_{1}\|\phi\|_{-1}\|\phi\|_{0}
$$

for all $\phi \in C_{0}^{\infty}\left(R^{n}\right)$. We have that $\operatorname{Re}(A \phi, \phi)_{0}=\left(\frac{1}{2}\left(A+A^{*}\right) \phi, \phi\right)_{0}$. However, since $\frac{1}{2}\left(A+A^{*}\right)=\mathscr{R}+\frac{1}{2}\left(A^{*}-A^{\#}\right)$, where $\mathscr{R}=(\operatorname{Re} a)(x, D)$ we obtain

$$
\operatorname{Re}(A \phi, \phi)_{0} \geqq \operatorname{Re}(\mathscr{R} \phi, \phi)_{0}-C_{2}\|\phi\|_{-1}\|\phi\|_{0} .
$$

Since $\mathscr{R} \phi=B \phi+\left(\lambda_{0}-\varepsilon / 2\right) \phi$ we have that $(\mathscr{R} \phi, \phi)_{0}=(B \phi, \phi)_{0}+\left(\lambda_{0}-\varepsilon / 2\right)\|\phi\|_{0}^{2}$. Using (8.1) and (8.2) we obtain

$$
\operatorname{Re}(A \phi, \phi)_{0} \geqq-C^{\prime}\|\phi\|_{-1}\|\phi\|_{0}+\left(\lambda_{0}-\varepsilon / 2\right)\|\phi\|_{0}^{2} .
$$

Taking

$$
\|\phi\|_{-1}\|\phi\|_{0} \leqq\left(\varepsilon / 2 C^{\prime}\right)\|\phi\|_{0}^{2}+C(\varepsilon)\|\phi\|_{-1}^{2}
$$

for all $\phi \in C_{0}^{\infty}\left(R^{n}\right)$ we are done.

Proof of Proposition 2. Let $A(t) \equiv(\eta+\varepsilon)^{2}-K(t)^{*} K(t)$. Then $A(t)=(\eta+\varepsilon)^{2}$

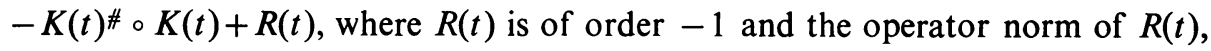
as a bounded mapping of $H^{-1}$ into $H^{0}$, is by Proposition 1, bounded by a constant $C_{1}$ independent of $t \in R^{1}$. Let $b(x, t ; \xi)=(\eta+\varepsilon)^{2}-|k(x, t ; \xi)|^{2}$; clearly $b(x, t ; \xi)$ 
satisfies condition (3.4) and $b(x, t ; \xi) \geqq \varepsilon^{2}$. Let $B(t)=b(x, t ; D)$; by our lemma we have that for any $\delta>0$ there is $C(\delta)>0$ such that

$$
\operatorname{Re}(B(t) \phi(t), \phi(t))_{0} \geqq\left(\varepsilon^{2}-\delta\right)\|\phi(t)\|_{0}^{2}-C(\delta)\|\phi(t)\|_{-1}^{2}
$$

for all $t \in R^{1}$ and $\phi \in C_{0}^{\infty}\left(R^{n+1}\right)$. Also, for arbitrary $\delta>0$,

$$
\left|(R(t) \phi(t), \phi(t))_{0}\right| \leqq \delta\|\phi(t)\|_{0}^{2}+C(\delta)\|\phi(t)\|_{0}^{2}
$$

for all $t \in R^{1}$. Since $A(t)=B(t)+R(t)$ we have that

$$
\operatorname{Re}(A(t) \phi(t), \phi(t))_{0} \geqq\left(\varepsilon^{2}-2 \delta\right)\|\phi(t)\|_{0}^{2}-C(\delta)\|\phi(t)\|_{-1}^{2}
$$

for all $t \in R^{1}$. However, since

$$
(A(t) \phi(t), \phi(t))_{0}=(\eta+\varepsilon)^{2}\|\phi(t)\|_{0}^{2}-\|K(t) \phi(t)\|_{0}^{2},
$$

we can take $\delta=\varepsilon / 2$ and we obtain our desired result.

Proof of Proposition 3. We shall employ the well-known fact that $u \in H^{-k}$ if and only if there exists $u_{0}, u_{1}, \ldots, u_{n} \in H^{-k+1}$ such that $u=u_{0}+\sum_{j=1}^{n} D_{j} u_{j}$ $\left(D_{j}=(1 / i) \partial / \partial x_{j}\right)$ and $C^{\prime}\|u\|_{-1}^{2} \leqq \sum_{j=0}^{m}\left\|u_{j}\right\|_{0}^{2} \leqq C^{\prime \prime}\|u\|_{-1}^{2}$. Since

$$
\zeta_{i} u=\left\{\zeta_{i} u_{0}-\sum_{j=1}^{n}\left(D_{j} \zeta_{i}\right) u_{j}+\sum_{j=1}^{n} D_{j}\left(\zeta_{i} u_{j}\right)\right\}
$$

we obtain

$$
\left\|\zeta_{i} u\right\|_{-1}^{2} \leqq C_{1}\left\{\left\|\zeta_{i} u\right\|_{0}^{2}+\sum_{j=1}^{n}\left\|\left(D_{j} \zeta_{i}\right) u_{j}\right\|_{0}^{2}+\sum_{j=1}^{n}\left\|\zeta_{i} u_{j}\right\|_{0}^{2}\right\}
$$

and

$$
\sum_{i}\left\|\zeta_{i} u\right\|_{-1}^{2} \leqq C \sum_{j=0}^{n}\left\|u_{j}\right\|_{0}^{2} \leqq C\|u\|_{-1}^{2}
$$

where

$$
C=C_{1} \sum_{|\alpha| \leqq 1} \sup _{x \in R^{n}} \sum_{i}\left|D^{\alpha} \zeta_{i}(x)\right|^{2} .
$$

We now assume the proposition holds for $k=1, \ldots, l-1$. There are constants $C^{\prime}, C^{\prime \prime}>0$ such that for each $u \in H^{-l}$ there exists $u_{0}, u_{1}, \ldots, u_{n} \in H^{-l+1}$ with $u=u_{0}+\sum_{j=1}^{n} D_{j} u_{j}$ and $C^{\prime}\|u\|_{-l}^{2} \leqq \sum_{j=0}^{n}\left\|u_{j}\right\|_{-l+1}^{2} \leqq C^{\prime \prime}\|u\|_{-l}^{2}$. Thus

$$
\left\|\zeta_{i} u\right\|_{-l}^{2}<C_{l}\left\{\left\|\zeta_{i} u_{0}\right\|_{-(l-1)}^{2}+\sum_{j=1}^{n}\left\|\left(D_{j} \zeta_{i}\right) u_{j}\right\|_{-(l-1)}^{2}+\sum_{j=1}^{n}\left\|\zeta_{i} u_{j}\right\|_{-(l-1)}^{2}\right\} .
$$

Applying our induction hypothesis we obtain

$$
\sum_{i}\left\|\zeta_{i} u\right\|_{-l}^{2} \leqq C \sum_{j=0}^{n}\left\|u_{j}\right\|_{-l+1}^{2} \leqq C\|u\|_{-l}^{2}
$$

where

$$
C=C_{k} \sum_{|\alpha| \leqq l} \sup _{x \in R^{n}} \sum_{i}\left|D^{\alpha} \zeta_{i}(x)\right|^{2},
$$

and our proof is complete. 
Proof of Proposition 6. First we assume $r=4 \mathrm{kp}$ and $s=0$ where $p$ is a nonnegative integer. In this case our proof will proceed by induction on $p$. For $p=0$ the result is immediate from (4.3) with $l=0$. Suppose now that our assertion is true for $p=0,1, \ldots, q-1$ where $q \geqq 1$, and suppose $u \in \mathscr{H}^{4 k q, 0}$ and $v \in \mathscr{H}^{-4 k q, 0}$. By Proposition 5, with $j=2$ and $p=q$, we know that $v$ can be expressed in the form $v=v_{0}$ $+\sum_{|\alpha|=4 k} D^{\alpha} v_{\alpha}+D_{t}^{2} v_{2}$, where $v_{0} \in \mathscr{H}^{-4 k(q-2), 0}, v_{\alpha} \in \mathscr{H}^{-4 k(q-1), 0}$ for $|\alpha|=4 k$ and $v_{2} \in \mathscr{H}^{-4 k(q-1), 0}$, in such a way that $\|v\|_{-4 k q, 0}$ is equivalent to

Thus

$$
\left\{\left\|v_{0}\right\|_{-4 k(q-2), 0}^{2}+\sum_{|\alpha|=4 k}\left\|v_{\alpha}\right\|_{-4 k(q-1), 0}^{2}+\left\|v_{2}\right\|_{-4 k(q-1), 0}^{2}\right\}^{1 / 2} \text {. }
$$

$$
\left[\zeta_{i} u, \rho_{i} v\right]=\left[\zeta_{i} u, \rho_{i} v_{0}\right]+\sum_{|\alpha|=4 k}\left[\zeta_{i} u, \rho_{i} D^{\alpha} v_{\alpha}\right]+\left[\zeta_{i} u, \rho_{i} D_{t}^{2} v_{2}\right]
$$

Since by our induction hypothesis, $\sum_{i}\left[\zeta_{i} \cdot, \rho_{i} \cdot\right]$ is continuous on $\mathscr{H}^{4 k(q-2), 0}$ $\times \mathscr{H}^{-4 k(q-2), 0}$, we obtain

$$
\left|\sum_{i}\left[\zeta_{i} u, \rho_{i} v\right]\right| \leqq C\|u\|_{4 k(q-2), 0}\left\|v_{0}\right\|_{-4 k(q-2), 0}
$$

where $C>0$ depends upon $\left\{\zeta_{i}\right\}_{1}^{\infty},\left\{\rho_{i}\right\}_{1}^{\infty}$, and $q$. Now for each $\alpha,|\alpha|=k$, consecutive integrations by parts yield

$$
\left[\zeta_{i} u, \rho_{i} D^{\alpha} v_{\alpha}\right]=\sum C_{\delta, \beta, \gamma}\left[\left(D^{\alpha} \zeta_{i}\right) D^{\beta} u,\left(D^{\gamma} \rho_{i}\right) v_{\alpha}\right],
$$

the sum being taken over a finite number of multi-indices $\delta, \beta$ and $\gamma$ with $\max \{|\delta|,|\beta|,|\gamma|\} \leqq 4 k$ and $\left|C_{\delta, \beta, \gamma}\right|=1$. By our induction hypothesis and condition (4.2) we have

$$
\begin{aligned}
\left|\sum_{i}\left[\left(D^{\delta} \zeta_{i}\right) D^{\beta} u,\left(D^{\gamma} \psi_{i}\right) v_{\alpha}\right]\right| & \\
& \leqq C\left\|D^{\beta} u\right\|_{4 k(q-1), 0}\left\|v_{\alpha}\right\|_{-4 k(q-1), 0} \leqq C\|u\|_{4 k q, 0}\left\|v_{\alpha}\right\|_{-4 k(q-1), 0}
\end{aligned}
$$

Thus

$$
\left|\sum_{i} \sum_{|\alpha|=4 k}\left[\zeta_{i} u, \rho_{i} D^{\alpha} v_{\alpha}\right]\right| \leqq C\|u\|_{4 k q, 0} \sum_{|\alpha|=4 k}\left\|v_{\alpha}\right\|_{-4 k(q-1), 0} .
$$

A similar procedure yields

$$
\left|\sum_{i}\left[\zeta_{i} u, \rho_{i} D_{t}^{2} v_{2}\right]\right| \leqq C\|u\|_{4 k q, 0}\|v\|_{-4 k q, 0}
$$

where $C$ depends upon $\left\{\zeta_{i}\right\}_{1}^{\infty},\left\{\rho_{i}\right\}_{1}^{\infty}$ and $q$.

For general $r$ and $s$, we employ the multilinear interpolation theorem [2]. Observe that, in the notation of [2], $\mathscr{H}^{r, s}=\left[\mathscr{H}^{r_{1}, s_{1}}, \mathscr{H}^{r_{2}, s_{2}}\right]^{\theta}$ where $r=(1-\theta) r_{1}+\theta r_{2}$, $s=(1-\theta) s_{1}+\theta s_{2}$ and $\theta \in[0,1]$. Given any real $r$ and $s$, we may write $r=2 k\left(p+\theta_{1}\right)$ and $s=l+\theta_{2}$, where $p$ and $l$ are integers, and $0 \leqq \theta_{1}, \theta_{2}<1$. Then since

$$
\begin{aligned}
\mathscr{H}^{r, s} & =\left[\mathscr{H}^{2 r, 0}, \mathscr{H}^{0,2 s}\right]^{1 / 2}, & \mathscr{H}^{-r,-s} & =\left[\mathscr{H}^{-2 r, 0}, \mathscr{H}^{0,-2 s}\right]^{1 / 2}, \\
\mathscr{H}^{2 r, 0} & =\left[\mathscr{H}^{4 k p, 0}, \mathscr{H}^{4 k(p+1), 0}\right]^{\theta_{1}}, & \mathscr{H}^{-2 r, 0} & =\left[\mathscr{H}^{-4 k p, 0}, \mathscr{H}^{-4 k(p+1), 0}\right]^{\theta_{1}}, \\
\mathscr{H}^{0,2 s} & =\left[\mathscr{H}^{0,2 l}, \mathscr{H}^{0,2(l+1)}\right]^{\theta_{2}}, & \mathscr{H}^{0,-2 s} & =\left[\mathscr{H}^{0,2 l}, \mathscr{H}^{0,-2(l+1)}\right]^{\theta_{2}},
\end{aligned}
$$

our proof is complete. 
AUtHOR's Note: Many of the techniques employed in this paper can be substantially improved by the use of pseudodifferential operators. In particular, the gap in Theorem 7 ( $r$ not an odd multiple of $k$ ) can be eliminated. These improvements will be presented in a separate paper.

\section{REFERENCES}

1. S. Agmon, Lectures on elliptic boundary value problems, Van Nostrand Math. Studies, no. 2, Van Nostrand, Princeton, N. J., 1965. MR 31 \#2504.

2. A. Calderón, Intermediate spaces and interpolation, the complex method, Studia Math. 24 (1964), 113-190. MR 29 \#5097.

3. - Singular integrals, Bull. Amer. Math. Soc. 72 (1966), 427-465. MR 35 \#813.

4. L. Hörmander, Linear partial differential operators, Die Grundlehren der math. Wissenschaften, Band 116, Academic Press, New York; Springer-Verlag, Berlin, 1963. MR 28 \#4221.

5. S. Kaplan, An analogue of Gdrding's inequality for parabolic operators and its applications, J. Math. Mech. 19 (1969), 171-188.

6. J. J. Köhn and L. Nirenberg, An algebra of pseudo-differential operators, Comm. Pure Appl. Math. 18 (1965), 269-305. MR 31 \#636.

7. B. Malgrange, Unicité du problème de Cauchy. Division des distributions, Séminaire Schwartz 4e année 1959/60, Faculté des Sciences, Paris, 1960, pp. 1-44. MR 23 \#A2275.

8. G. E. Silov, Mathematical analysis: Second special course, "Nauka”, Moscow, 1965; English transl., Generalized functions and partial differential equations, Gordon and Breach, New York, 1968. MR 36 \#2943; MR 37 \#5694.

Department of Mathematics, Herbert H. Lehman College (CUNY), Bronx, New York 10468 